\title{
Code of Practice and recommendations for Total Body Irradiation and Total Skin Irradiation
}

\author{
NEDERLANDSE COMMISSIE VOOR STRALINGSDOSIMETRIE
}

Report 34 of the Netherlands Commission on Radiation Dosimetry February 2021 


\section{Disclaimer regarding NCS reports}

The NCS frequently publishes reports for fellow professionals in which recommendations are given for various quality control procedures or otherwise. The members of the NCS board and the members of the concerning subcommittee do not claim any authority exceeding that of their professional expertise. Responsibility on how the NCS recommendations are implemented lies with the user, taking into account the practice in his/her institution.

This report may identify certain commercial equipment, instruments, or materials to specify the experimental procedure adequately. Such identification does not imply recommendation or endorsement, nor that the materials or equipment identified are necessarily the best available for the purpose.

\section{Terminology in this report}

The following levels of recommendation are used throughout this report:

- "must" means there is a legal obligation according to Dutch and/or Belgian law or formal communication from the government;

- "should" indicates a strong recommendation. Not abiding to this recommendation needs to be motivated and documented, along with a description of an adequate alternative method to cope with the issue at hand;

- "recommend" or "advise" means a mere suggestion. This recommendation may be disregarded, keeping in mind that there is a reason for mentioning it in the report.

- "local protocol" means that there should be a clear, written protocol on how to check that particular item, including tolerance/action levels and the person responsible for performing the check(s).

The recommendations in NCS reports aim to optimise the treatment or diagnosis procedure by optimising QA procedures. Still, the reader should be aware that safety recommendations as described elsewhere, for instance by manufacturers, still need to be considered. In general, NCS and other recommendations should be taken seriously notwithstanding careful and thorough thought. 


\section{Preface}

The Nederlandse Commissie voor Stralingsdosimetrie (NCS, Netherlands Commission on Radiation Dosimetry, http://www.radiationdosimetry.org) was officially established on 3rd September, 1982 with the aim of promoting the appropriate use of dosimetry of ionising radiation both for scientific research and for practical applications. The NCS is chaired by a board of scientists, made up via recommendations from the supporting societies, including the Nederlandse Vereniging voor Radiotherapie en Oncologie (Dutch Society for Radiotherapy and Oncology), the Nederlandse Vereniging voor Nucleaire Geneeskunde (Dutch Society of Nuclear Medicine), the Nederlandse Vereniging voor Klinische Fysica (Society for Medical Physics of the Netherlands), the Nederlandse Vereniging voor Radiobiologie (Netherlands Radiobiological Society), the Nederlandse Vereniging voor Stralingshygiëne (Netherlands Society for Radiological Protection), the Nederlandse Vereniging voor Medische Beeldvorming en Radiotherapie (Dutch Society for Medical Imaging and Radiotherapy), the Nederlandse Vereniging van Klinisch Fysisch Medewerkers (Dutch Society for Medical Physics Engineers), the Nederlandse Vereniging voor Radiologie (Radiological Society of the Netherlands) and the Belgische Vereniging voor Ziekenhuisfysici/Société Belge des Physiciens des Hôpitaux (Belgian Hospital Physicists Association) and expanded with a representative from the Dutch Metrology Institute VSL. To achieve its aims, the NCS carries out the following tasks: participation in dosimetry standardisation, promotion of mutual comparisons of dosimetry, drafting of dosimetry protocols and the collection and evaluation of physical data related to dosimetry. Furthermore, the commission shall establish or maintain links with national and international organisations concerned with ionising radiation and promulgate information on new developments in the field of radiation dosimetry.

Current members of the board of the NCS

J.B. van de Kamer, Chairman

T.W.M. Grimbergen, Vice-Chairman

J.A. de Pooter, Secretary

J.M.J. Hermans, Treasurer

G. Pittomvils

N. De Graaf

F.W. Wittkämper

M.K. de Fluiter-Zeeman/E. van de Zande

J.R. de Jong

F. Dekkers 


\section{Code of Practice and recommendations for Total Body Irradiation and Total Skin Irradiation}

Members of the subcommittee:

Lars Murrer

Peter van der Hulst

Wim Jansen

Ruud van Leeuwen

Phil Koken

Damien Dumont

Laurien Daniëls

Jeroen van de Kamer

Geert Pittomvils

NCS, Delft, the Netherlands

For more information on NCS Reports, see http://radiationdosimetry.org 


\section{Summary}

In October 2014, the NCS installed a new subcommittee on the code of practice and the recommendations for Total Body Irradiation (TBI) and Total Skin Irradiation (TSI) treatments. As a first step this subcommittee investigated the status of the treatment protocols and quality assurance for TBI and TSI treatments in Belgium and the Netherlands. The results of this survey confirmed our expectation: despite many decades of experience and previous surveys, a large variety remained in treatment protocols, being a combination of evolved traditional techniques and more modern CTbased irradiation techniques.

There were several reasons to draft this report: 0) To provide a guideline for institutes that are considering starting up TBI/TSI treatments; 1) standardisation of the actual manual calculation workflow; 2) creation of a reference in order to benchmark the individual institution protocol allowing guided improvement; 3 ) description of novel techniques already developed in centres of excellence in order to guide and accelerate the evolution towards new treatment techniques in Belgium and the Netherlands.

This report is a step in the process in the evolution to a state-of-the-art treatment technique. This report does not develop any new techniques. Instead, it describes current practiced techniques and possible guidelines to implement such techniques clinically.

DOI: $10.25030 /$ ncs-034 


\section{Abbreviations and acronyms}

\begin{tabular}{|c|c|}
\hline AAPM & American Association of Physicists in Medicine \\
\hline AMDEC & Analyse des Modes de Défaillance, de leurs Effets et de leur Criticité \\
\hline AML & Acute Myeloid Leukaemia \\
\hline AP-PA & Anterior Posterior-Posterior Anterior \\
\hline BIV & Beschikbaarheid, integriteit en vertrouwelijkheid \\
\hline CCD & Charged Coupled Device \\
\hline CIED & Cardiac Implantable Electronic Device \\
\hline CT & Computed Tomography \\
\hline DICOM & \\
\hline RTplan & Digital Imaging and COmmunications RadioTherapy Plan \\
\hline DRR & Digitally Reconstructed Radiograph \\
\hline DVH & Dose-Volume Histogram \\
\hline EBT films & External Beam Therapy Films \\
\hline FWHM & Full Width-Half Maximum \\
\hline GDPR & European General Data Protection Regulation \\
\hline GT & Gun Target \\
\hline GvHD & Graft-versus-Host Disease \\
\hline GvL & Graft-versus-Lymphoma \\
\hline HFMEA & Healthcare Failure Mode and Effect Analysis \\
\hline HFP & Head First Prone \\
\hline HFS & Head Fist Supine \\
\hline HSCT & Hematopoietic Stem Cell Transplant \\
\hline HVL & Half-Value Layer \\
\hline IMRT & Intensity-Modulated Radiation Therapy \\
\hline $\mathrm{MeV}$ & Mega-electronVolt \\
\hline MLC & MultiLeaf Collimator \\
\hline MLD & Mean Lung Dose \\
\hline MOSFET & Metal-Oxide-Semiconductor Field-Effect Transistor \\
\hline $\mathrm{MU}$ & Monitor Unit \\
\hline MV & MegaVolt \\
\hline NACP & Nordic Association of Clinical Physicists \\
\hline NCS & Nederlandse Commissie voor Stralingsdosimetrie \\
\hline $\mathrm{NHL}$ & Non-Hodgkin Lymphoma \\
\hline OAR & Organ-At-Risk \\
\hline PA & Posterior-Anterior \\
\hline PDD & Percentage Depth-Dose curve \\
\hline PIA & Privacy Impact Assessment \\
\hline PMMA & Poly(Methyl MethAcrylate) \\
\hline PTV & Planning Target Volume \\
\hline QA & Quality Assurance \\
\hline$R \& V$ & Record and Verify \\
\hline RTT & Radiation Therapy Technologist \\
\hline
\end{tabular}




$\begin{array}{ll}\text { SAD } & \text { Source-to-Axis Distance } \\ \text { SAFER } & \text { Scenario Analyse van Faalwijzen, Effecten en Risico's } \\ \text { SSD } & \text { Source-to-Surface Distance } \\ \text { TBI } & \text { Total Body Irradiation } \\ \text { TLD } & \text { Thermo Luminescence Dosimeter } \\ \text { TMR } & \text { Tissue-Maximum Ratio } \\ \text { TPR } & \text { Tissue-Phantom-Ratio } \\ \text { TPS } & \text { Treatment Planning System } \\ \text { TSI } & \text { Total Skin Irradiation } \\ \text { VMAT } & \text { Volumetric Modulated Arc Therapy }\end{array}$




\section{Contents}

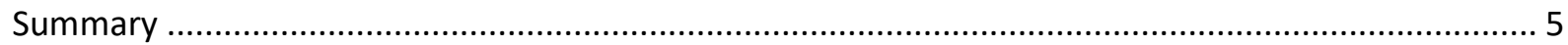

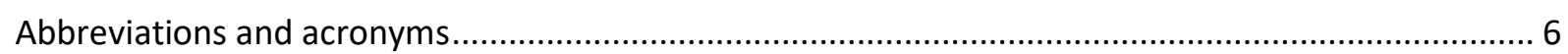

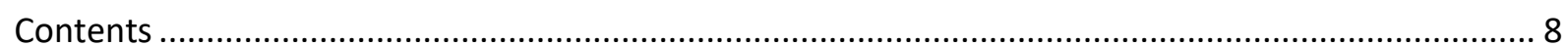

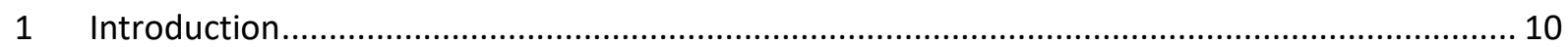

2 Clinical requirements for total body irradiation and total skin irradiation ................................. 11

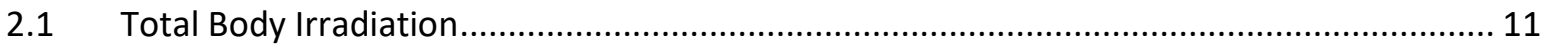

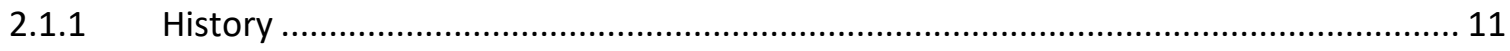

2.1.2 Acute side effects of TBI treatment ……................................................................. 13

2.1.3 Late side effects of TBI treatment .............................................................................. 14

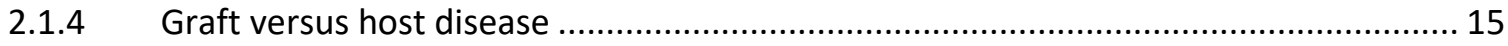

2.1.5 Technical aspects of TBI from a clinical perspective ................................................ 15

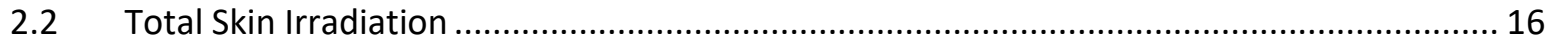

3 Total Body Irradiation and Total Skin Irradiation techniques in Belgium and the Netherlands -

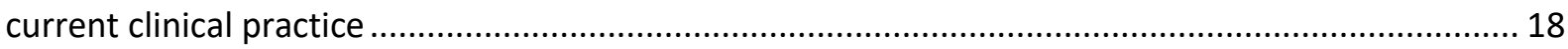

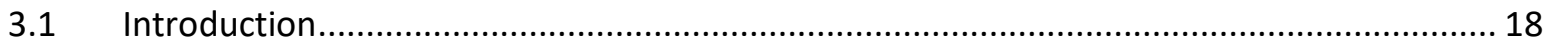

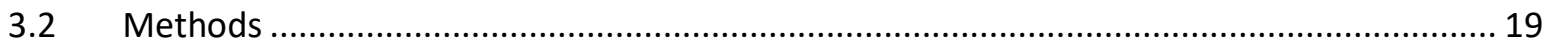

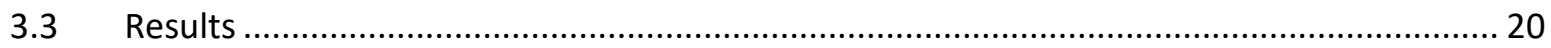

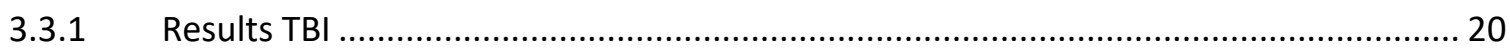

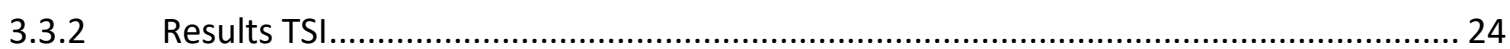

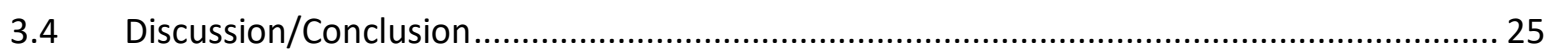

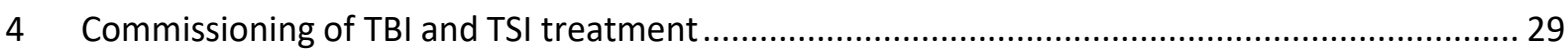

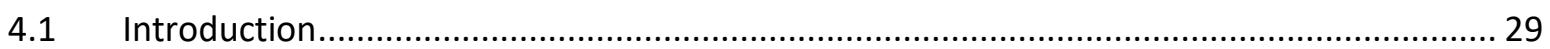

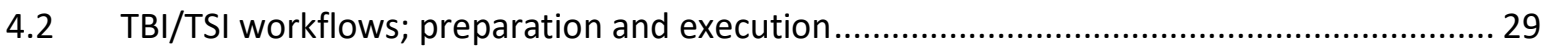

4.2.1 The manual calculation workflow: procedure without using a treatment planning

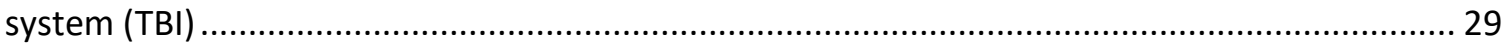

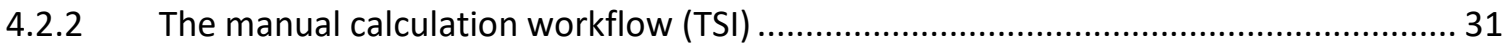

4.2.3 The TPS workflow: procedure including full integration of a TPS (TBI)....................... 31

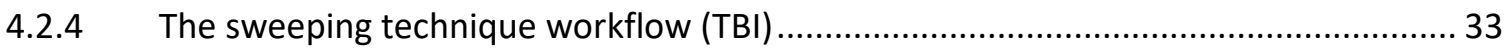

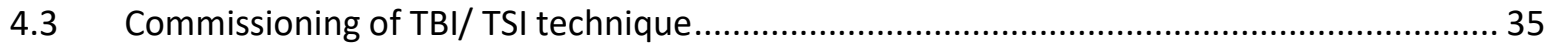

4.3.1 Absolute beam calibration in TBI/TSI-reference setup (TBI/TSI) ................................ 35

4.3.2 Beam Flatness (beam profiles i.e. off-axis ratios) at TBI/TSI-distance (TBI/TSI) ........... 38 


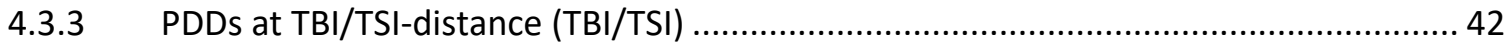

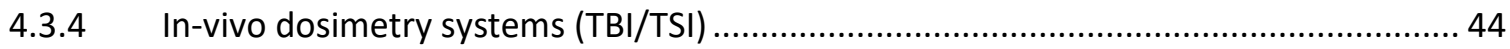

4.3.5 Dry runs/end-to-end test and in-vivo dosimetry (TBI/TSI) .................................45

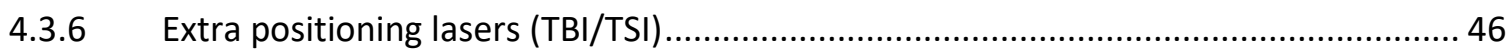

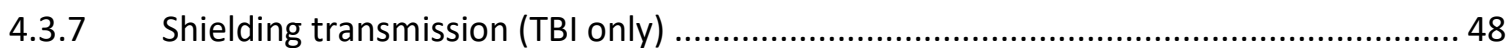

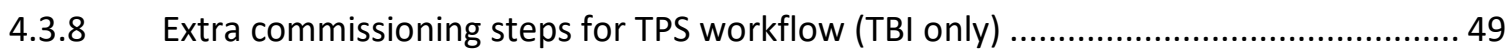

4.3.9 Extra measurements for sweeping techniques (TBI only)..................................51

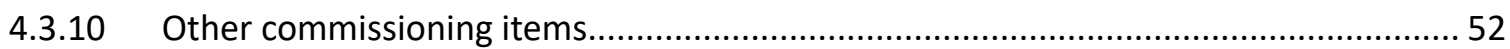

4.3.11 Cardiac Implantable Electronic Device and other electronic devices (TBI/TSI) ........... 53

5 Pre-treatment workflow, treatment delivery and in-vivo verification .....................................5 54

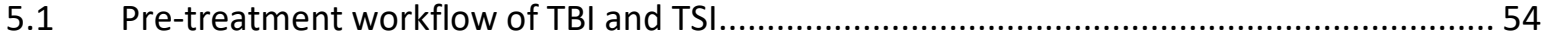

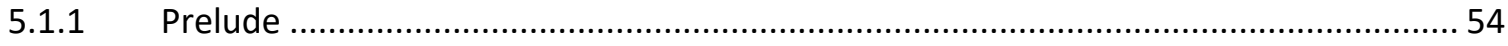

5.1.2 The manual calculation workflow ............................................................. 54

5.1.3 Transfer of shielding parameters to the block-milling machine for TBI treatments..... 56

5.1.4 Shielding block transmission and compensator transmission under TBI-conditions.... 56

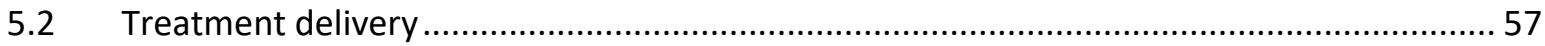

5.2.1 Recommendations for single and multiple fraction treatment delivery for TBI/TSI.... 57

5.2.2 Recommendation for treatment verification in multiple fraction treatment delivery. 58

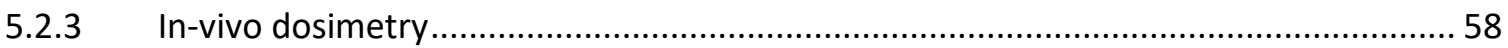

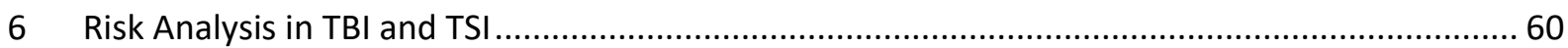

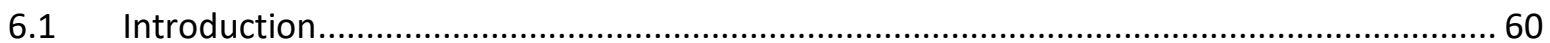

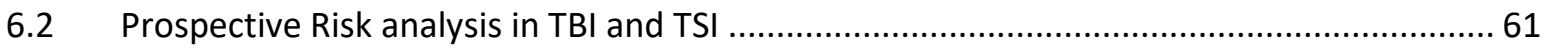

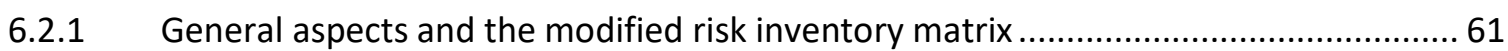

6.2.2 Examples of failure modes in the TBI/TSI development and introduction ..................63

6.2.3 Examples of failure modes in the TBI clinical workflow ........................................63

Examples of failure modes in the TSI clinical workflow ......................................69 69

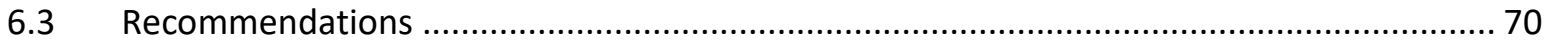

$7 \quad$ Final words and some remarks about future developments ............................................... 72

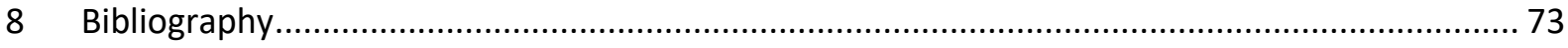

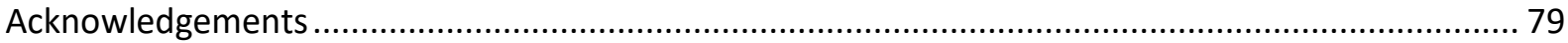




\section{Introduction}

In October 2014, the NCS installed a new subcommittee on the code of practice and the recommendations for Total Body Irradiation (TBI) and Total Skin Irradiation (TSI) treatments.

In this report the authors will give some recommendations on the quality assurance process for Total Body and Total Skin Irradiation treatments. While those techniques differ significantly to standard irradiation techniques and while the clinical requirements are very specific a more clinical description of the needed requirements is reported in chapter 2.

The third chapter gives an overview of the results of the questionnaire send around in 2017, a chapter that stands self-contained, using the standard scientific lay-out as it is submitted for publication. The fourth chapter describes the point of view, based on experience of the commissioning process including pitfalls for the different workflows in use in Belgium and the Netherlands. Due to the specificity and the low frequency of treatments, extra attention should be paid to the pre-treatment workflow and the treatment delivery. This is discussed in chapter 5 . This specificity and low frequency have also a large impact on the risks involved. The aim of chapter 6 of this report is to help each institute to draw up their own risk analysis in order to improve specific parts of their workflow. The report ends with a projection of the evolution towards a state of the art TBI/TSI treatment technique. 


\section{Clinical requirements for total body irradiation and total skin irradiation}

\subsection{Total Body Irradiation}

In radiation oncology we commonly define our target volume as a tumour, surrounded by appropriate margins to account for multiple clinical and physical uncertainties, e.g. microscopic tumour spread, delineation variation, and tumour and patient mobility. However, in the treatment of some hematologic malignancies, the target volume may comprise the entire hematopoietic system, which corresponds to the entire body.

The first experiences with a total body irradiation (TBI) date from the early 1900s, when Friedrich Dessauer and Aladar Elfer described their experiences in treating leukaemia patients with this new radiation technique [1-3]. In the following decades refinement in treatment techniques and patient selection were made.

\subsubsection{History}

The first report of a large series of patients in 1942 showed superior response rate of TBI in those with hematopoietic and lymphoid malignancies compared to solid tumours. It also made the first recommendations concerning safe treatment doses with tolerable toxicity [4].

During the second world war military interest emerged in the United States in the effect of ionizing radiation on humans, because of the development of nuclear weapons [5]. This incentivised the development of various research projects. Clinical research financed by the Department of Defense of the USA investigated dosimetric effects but also the physical and neurocognitive side effects of treatment [6-8]. At that time, patients were often included in these studies without informed consent, unfortunately [9].

In the 1950s the first report of the use of TBI as an immunosuppressive regimen prior to organ transplant as opposed to treatment for (advanced) malignant diseases was published [10]. Nowadays, immunosuppression in organ transplant medicine is accomplished by alternative immunosuppressive agents given after transplantation [11]. However, the immunosuppressive effect through TBI remains an important aspect of treatment of hematopoietic diseases.

By the end of the 1950s Donnall Thomas reported his experiences with TBI as a successful conditioning regimen before hematopoietic bone marrow infusions, although no durable remissions were obtained $[12,13]$. Major challenges in the time period before engraftment of the 
transplanted cells were myelosuppression with associated infections and bleeding risks [13]. Several different approaches in terms of both dose rate and fractionation were investigated. The first reported sustained remission of a patient with leukaemia treated with TBI using opposing ${ }^{60} \mathrm{Co}$ sources to a calculated midline tissue dose of 954 rads, dates from 1969 [14].

Over the past decade's refinements in the TBI techniques, dose, and fractionation have been made. The combination of TBI with improved systemic treatment offered curative treatment options for patients even with poor prognosis or refractory disease $[15,16]$.

Nowadays the use of TBI is provided only in combination with hematopoietic stem cell transplant (HSCT), in conjunction with systemic treatment. Very rarely TBI is used as a single modality for the (palliative) treatment of chemotherapy-refractory leukaemia's and lymphomas.

There are two types of HSCT. One is an autologous stem cell transplant where the body's own stem cells are re-infused after high dose systemic treatment. A second type is an allogeneic stem cell transplant where stem cells from a related or unrelated matched donor are administered after appropriate conditioning which can be either myeloablative (meaning that no autologous hematopoietic recovery is possible) or non-myeloablative, frequently called reduced intensity conditioning (RIC) [5].

Whether an autologous or allogeneic HSCT is performed depends on multiple factors. This includes the type of underlying hematologic disease and previous treatments but also age, comorbidity and performance status. It also depends on whether or not a donor is available.

The conditioning regimen for an autologous transplant consists of high-dose systemic therapy. The objective of this treatment is to aggressively treat all residual lymphoproliferative cells. This, however, results in irreversible damage to the bone marrow. The patient's own stem cells that were harvested at an earlier stage are then given back to restore the hematopoietic cell lines. An autologous transplantation is typically considered in patients with multiple myeloma, certain types of Non-Hodgkin lymphoma (NHL) such as T-cell lymphomas or mantle cell lymphomas, acute myeloid leukaemia (AML) when no allogeneic donor is found, and in the setting of recurrent aggressive NHL or Hodgkin lymphoma [17].

The conditioning regimen for an allogeneic HSCT consists of systemic therapy with or without TBI, resulting in both a direct anti-tumour effect and eradication of the own haematopoietic system of 
the host. However, there is also an additional immunologic anti-tumour effect. This consists of the detection of potential residual tumour cells by the donor's immune cells, the so-called graft versus lymphoma $(\mathrm{GvL})$ effect. An allogeneic transplantation is considered in patients with poor prognosis acute myeloid leukaemia (AML) or acute lymphoblastic leukaemia, rescue treatment in some types of chemo-refractory NHL, aplastic anaemia, and (rarely) in patients with chronic lymphocytic leukaemia $[18,19]$.

The conditioning regimen for an autologous HSCT is always myeloablative. The conditioning regimen for an (allogeneic) HSCT can be either myeloablative or non-myeloablative, depending on factors previously mentioned.

In a non-myeloablative or RIC regimen a lower dose of systemic treatment with or without lowdose TBI is used. This means a TBI dose of $\leq 5 \mathrm{~Gy}$ in a single fraction (usually $1 \times 2$ Gy or 1 x 3 Gy) or $\leq 8 \mathrm{~Gy}$ fractionated dose (usually $2 \times 2 \mathrm{~Gy}$ ) [20]. The goal of the conditioning regimen is to achieve an immunosuppressive effect, to ensure the engraftment of donor stem cells to achieve a GvL effect. In a myeloablative conditioning regimen a (high dose of) systemic treatment is given, sometimes combined with a high dose in a single fraction or fractionated TBI (total dose typically 8-16 Gy), mainly in the setting of an allogeneic HSCT for a lymphatic malignancy. The advantage of the use of TBI in combination with systemic treatment is that radiation therapy is not as dependent on blood supply as chemotherapy. Also, it can eradicate possible chemo-resistant tumour cells and can reach sanctuary sites such as testes and the central nervous system [20].

\subsubsection{Acute side effects of TBI treatment}

The acute toxicity of TBI can be difficult to distinguish from the toxicity of systemic treatment. It is also dependent on the total dose of radiation and whether or not it was administered as a single dose or fractionated.

The most common acute side effects are fever (>90\% single dose), nausea and vomiting $(80-90 \%$ single-fraction $\mathrm{TBI}, 20-40 \%$ fractioned TBI), painful and/or swollen parotid glands (75\% singlefraction $\mathrm{TBI}, 10 \%$ fractionated $\mathrm{TBI}$ ), xerostomia (60\% single-fraction $\mathrm{TBI}, 30 \%$ fractionated $\mathrm{TBI}$ ), and headaches (40\% single-fraction $\mathrm{TBI}, 15 \%$ fractionated $\mathrm{TBI}$ ). Mucositis, alopecia, radiation pneumonitis and erythema of the skin are also commonly reported side effects [5,21,22]. In general, acute side effects seem to be less common and severe in a fractionated scheme [23]. 


\subsubsection{Late side effects of TBI treatment}

The hematopoietic system is the most sensitive organ to radiation. Lymphopenia is seen shortly after TBI, even with low dose, followed by thrombocytopenia and ultimately anaemia [5]. The possibility of regeneration is dependent on the radiation dose used: a higher dose results in a more rapid myelosuppression but also in a slower recovery [5]. Stimulation of regeneration by using growth factors is associated with a higher risk of graft-versus-host disease (GvHD) (see below) [24]. Pneumopathy (e.g. interstitial pneumonitis, fibrosis) is the dose-limiting toxicity in the use of TBI [20]. The cause of pneumopathy is thought to be multifactorial: use of chemotherapy, sensitivity to pulmonary infections in often immune-compromised patients, and the occurrence of GvHD. It has been described in up to $20 \%$ of patients without TBI as part of their conditioning regime [25], and in $10-80 \%$ of patients with TBI $[20,26]$. A number of factors have been shown to influence the risk of pneumopathy, with first of all, the total dose, especially if this is higher than $10 \mathrm{~Gy}$ [26-30]. To be able to treat patients with a single myeloablative dose, shielding of the lung is performed. Fractionated TBI results in a lower incidence of pneumopathy mostly when the total dose exceeds $10 \mathrm{~Gy}$. Mixed results have been reported on dose rate. Preclinical animal studies have shown the effect of dose rate on bone marrow stem cells is limited, but normal tissue tolerance seems to be affected by it [31,32]. Clinical retrospective data has shown a benefit of using a low dose rate $(\sim<<10$ cGy/min) in preventing pneumopathy $[26,27]$. This, however, has not been confirmed in a prospective randomized trial, also due to the many confounding factors involved [23]. Given the limited evidence on this matter using high dose rate is current practice, also prompted by practical reasons (e.g. radiation treatment time and as a result patient positioning stability and patient comfort).

Cardiovascular toxicity after TBI is observed in about $2-3 \%$ of patients. This low percentage may be due to the fact that patients with cardiac risk factors or comorbidity are often ineligible to undergo HSCT $[33,34]$.

Other late treatment effects are persisting xerostomia (25-50\% of patients) both after singlefraction or fractionated TBI. Improvement in xerostomia can occur in the first year after treatment [5]. Thyroid dysfunction, hepatotoxicity (mostly as sinusoidal obstruction syndrome, formerly known as veno-occlusive disease), cataract and kidney dysfunction can also occur after treatment $[5,20]$. There is an increased risk of secondary malignancies, both hematological and solid tumours, predominantly in patients treated under the age of 30 [20].

Definition of the maximum tolerated dose in organs at risk is difficult, it is a complex balance of comorbidity, type of conditioning, fractionation and total radiation dose. Besides the lungs some 
institutions use extra shielding of for example eyes or kidneys in order to minimize the risk of late toxicity.

\subsubsection{Graft versus host disease}

Graft versus host disease (GvHD) occurs in $30-40 \%$ of patients with a related donor and in $60-80 \%$ of patient with an unrelated donor after an allogeneic stem cell transplant [35]. Where the donor immune cells have a function in detecting residual malignant cells (the so-called graft-versuslymphoma effect) they can also detect small differences from healthy cells resulting in an immune response to these healthy cells. GvHD can manifest itself in the skin, gastro-intestinal tract or liver (acute GvHD) or in the skin, mouth, eyes or lungs (chronic GvHD) [36]. Although its occurrence is not a result of the TBI (but related to the donor stem cells) it can increase the risk of radiationinduced toxicity or its severity [37].

\subsubsection{Technical aspects of TBI from a clinical perspective}

TBI differs from conventional external beam radiation treatment in several specific ways. Because it is a relatively rare treatment, a dedicated team of doctors, RTT's and physicists, preferably performs it.

To make sure the patient fits within the radiation beam, patients are usually treated at a larger distance from the linear accelerator than in conventional treatments. Modern radiotherapy techniques like rotational intensity-modulated radiotherapy are currently used in a very limited number of centers. The most frequently used technique is with opposing antero-posterior (AP) and postero-anterior (PA) fields with the patient in a decubitus position. Although an upright position is preferable from a dosimetric perspective because the cross-sectional differences are minimized in this position, it can be an uncomfortable position for the patient and often requires supports in order to avoid patient movements during the irradiation lasting 10 minutes or more. Another option is to use a sitting position [5,38].

Dose heterogeneity depends on the technique: the position used and the use of compensators or field-in-field MLC segments to homogenize the dose. The required dose homogeneity can be, e.g. $80-120 \%$ for the whole body. For the radiation treatment itself, megavoltage (MV) beams are used. Higher energy beams ( $\geq 10 \mathrm{MV}$ ) provide a more homogeneous plan; although this makes lung shielding less effective due to increased lateral scatter. In that case, the skin-sparing effect of higher energy beams is countered by using an acrylic scatter spoiler screen. Whether or not a scatter spoiler screen should be standard clinical practice remains a matter of dispute: some believe that 
circulating tumour cells can reside in the skin, making the skin part of the target area for TBI treatment $[19,20]$. Others believe the target structure for TBI is limited to (skeletal) bone marrow and use of spoilers merely add toxicity to the treatment and therefor promote the use of total marrow irradiation [20].

Timing of the TBI in relation to the transplantation depends on the type of conditioning (myeloablative or not) and the relation and type of match between donor and patient. Since the stem cells to be transplanted can only be stored for a limited period of time, to avoid loss of viability, postponement of the TBI treatment must be avoided.

\subsection{Total Skin Irradiation}

Total skin irradiation (TSI) or Total skin electron beam therapy is a treatment of the complete skin with superficial (electron beam) radiation therapy. The technique had been developed in the 1940s by Trump and colleagues as a treatment option for patients with extensive superficial malignant disease, particularly cutaneous T-cell lymphoma [39]. For these patients extended-field treatment with low-voltage $\mathrm{x}$-rays (with a deeper penetration) would result in unacceptable hematopoietic toxicity. The first patient was treated in 1952 with the use of a collimated electron beam generated by a Van der Graaff generator [39]. The original technique of four body rotations was further refined in Stanford with the use of a linear accelerator [40]. This Stanford technique is still most commonly in use, in more than $80 \%$ of the radiation therapy facilities that perform TSI [41]. It consists of a 15$20^{\circ}$ dual (fixed)-angle six-field method (see figure) to improve dose uniformity, with anterior, posterior and four oblique fields [42]. One of the advantages of this technique is that because of the dual angles it can be used even in smaller treatment facilities. Depending on the total number of fractions all six positions or only 3 out of 6 positions in an alternating way can be given each fraction [43]. Other, less frequently-used techniques are translational techniques, large electron field techniques using overlapping fields or rotational techniques [44].

The target volume for TSI includes the dermis, the epidermis and adnexal structures. Aim is to have a dose homogeneity between $85 \%$ and $115 \%$ of the prescribed dose on the skin, ranging from the surface until a depth of $4 \mathrm{~mm}$. This is achieved using either $4 \mathrm{MeV}$ electron beams without degraders or $6 \mathrm{MeV}$ with degraders. Photon contamination should be restricted to less than $0.7 \mathrm{~Gy}$ (total treatment) to avoid hematopoietic toxicity $[45,46]$.

Treatment with TSI is considered as a palliative treatment in patients with extensive disseminated skin lesions, either too thick for treatment with phototherapy or phototherapy-resistant, to relieve 
symptoms such as pain from (ulcerating) tumours or pruritus. Usually these will be patients with a cutaneous T-cell lymphoma such as mycosis fungoides [47]. In rare cases, (low-grade) cutaneous Bcell lymphomas affect a large skin surface although usually this will be limited to one or two extremities or the scalp. In this case, an adaptation in the treatment volume can be made by shielding parts of the body that are not affected.

Traditionally, patients have been treated with high-dose TSI, ranging from 30-36 Gy (usually 1.5-2.0 Gy per fraction), supported by a dose-effect relationship. With a radiation dose $>30$ Gy more than $80 \%$ of patients achieve a complete response and relief of severe symptoms is achieved in all patients $[48,49]$. The most commonly reported toxicity with a high dose TSI is generalized erythema of the skin, (temporary) hair loss, oedema of the feet, conjunctivitis when the eyes are not shielded and brittleness of the nails [50]. The duration of the response can vary widely, with a median of 12 months [51]. Response duration seems to be longer in lower stage disease [50]. In the past years however low-dose TSI (10-13 Gy, fraction size of 1-2 Gy, 2-3 times a week) has gained ground. Clear advantages of this low-dose regimen are that it is far better tolerated by patients, less toxic and can be repeated more often than a high-dose course [52]. However, the number of complete responses is less, as well as the response duration is markedly less with a low-dose treatment. Presently, both schemes are used in the Benelux.
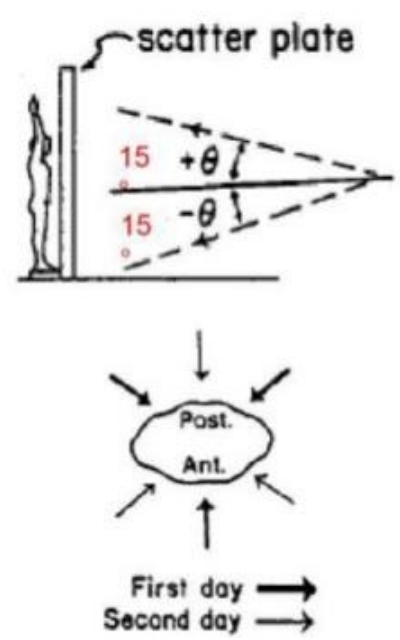

Figure 2.1 Stanford method of irradiation for total skin irradiation in axial view and sagittal view. 


\section{Total Body Irradiation and Total Skin Irradiation techniques in Belgium and the Netherlands - current clinical practice}

This chapter is accepted for publication in Advances in Radiation Oncology (2021) [53].

\section{Summary}

A Belgian/Dutch NCS task group developed guidelines on clinical practice of Total Body Irradiation (TBI) and Total Skin Irradiation (TSI), supported by a survey among participating institutions. As old and more recent studies showed, clinical protocols for TBI and TSI still vary considerably among institutions, showing that new radiotherapy technologies have been relatively slowly introduced for TBI/TSI purposes.

\section{Abstract}

Purpose: In 2014, a Belgian/Dutch NCS task group was formed to develop guidelines on clinical practice of Total Body Irradiation (TBI) and Total Skin Irradiation (TSI).

Methods and Materials: As a basis for these guidelines, a survey conducted among 17 Belgian and Dutch radiation oncology institutions measured clinical practice of TBI. Four of these institutions also performed TSI. An update was performed in 2019 and 2020 because several institutions innovated their TBI techniques.

Results: As old and more recent studies showed, clinical protocols for TBI and TSI still vary considerably among institutions.

Conclusion: New radiotherapy technologies have been introduced relatively slowly for TBI purposes.

\subsection{Introduction}

TBI and TSI have been practiced for over a century, and since about last mid-century, respectively. After Dessauer and Eifer practiced TBI in 1905 and 1907 [38,54] many others followed [55,56]. Despite the long-term clinical practice and experience, already three decades ago it has been shown that TBI techniques vary significantly over Europe [57] and Japan [58]. Quast [57] argued that survival rates depend primarily on indication and staging, but that variations in treatment techniques may also play a role. 
In the past 30 years techniques have not converged as multiple, recent publications showed. Studinski [59] showed that for TBI no commonly accepted planning and treatment delivery exists. Similar findings have been published recently in Japanese [60,61], European-Middle East [62], Australian $[63,64]$ and American studies $[65,66]$. TSI is much less performed clinically but, again, multiple approaches exist in treatment techniques [67].

Applying newer hard- and software, cutting-edge techniques for TBI were developed. Some of these (eg., VMAT based techniques) are already used clinically [68-71]. Other potential techniques are helical Tomotherapy [72-77], proton therapy [56], radio-immunotherapy $[78,79]$ or more complex VMAT techniques including couch rotations and high precision delineations of multiple structures once logistics have been optimized $[80,81]$.

In 2014, the NCS committee formed a Belgian/Dutch task group to develop guidelines on clinical practice of TBI and TSI, employing a survey held among Belgian and Dutch radiotherapy institutions. An update was performed in 2020 because several institutions innovated their TBI techniques.

Compared to recent published surveys, more detailed questions about TBI were included, and (to our knowledge, for the first time) TSI was surveyed as well. The results are presented and discussed in this report.

\subsection{Methods}

A survey was performed electronically by all Dutch and Belgian radiation oncology institutions in 2014 . The survey consisted of 5 general, 49 TBI-related and 38 TSI-related questions (Questionnaire: see DOI: $10.25030 /$ ncs-034).

According to the Dutch cancer registry, the incidence of hematological malignancies in the Netherlands is 9540 in 2018 [82] with approximately 310 TBI treatments annually. Belgium registries [83] report incidences of about 6500 and 136 TBI treatments annually. The Netherlands reported 15 annual TSI treatments in the questionnaire (incidence of mycosis fungoides: 100/year; 17 million inhabitants), and Belgium 11 (incidence 47/year; 10.4 million inhabitants).

Data were processed electronically and wherever necessary responders were encouraged to response in detail. Our goal was met to achieve $100 \%$ response to get a complete overview of the clinical practice 
regarding TBI and TSI in the Netherlands and Belgium. The results shown in this report represent the status of 2020.

\subsection{Results}

All 21 Dutch radiation oncology institutions responded. In total nine Dutch institutions practice TBI, including all eight university centers. Only one (university) institution practices TSI. In total 10 Belgian institutions responded; eight of them, including seven academic institutions, practice TBI. Three of the (university) centers that perform TBI also perform TSI.

Results from both countries are grouped, unless indicated otherwise. Because the survey was not always fully completed by every single institution, statistics are processed and quoted accordingly (i.e. numbers of institutions may vary over the answered questions).

\subsubsection{Results TBI}

Per annum on average 26 TBI patients are treated per institution (average 25 , range 1-70) for all dose schemes. See also Figure 3.1.

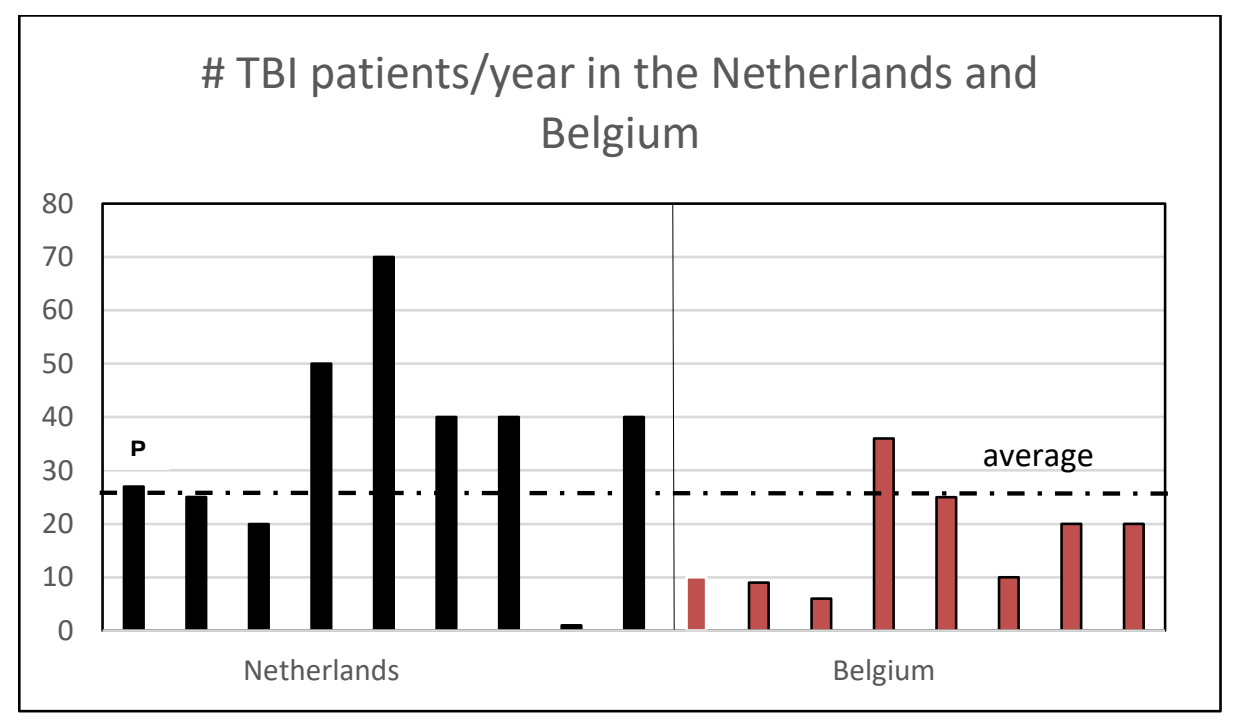

Figure 3.1. Number of TBI patients treated annually in the Netherlands (black bars) and Belgium (red bars). Each bar represents an institution. Average value indicates average number of TBI patients annually for both countries. ' $P$ ' = dedicated pediatric treatment center.

The reported dose-fractionation schemes show a large variety in total dose and fractionation strategy (seeTable 3.1). For non-ablative treatments $1 \times 2$ Gy and $2 \times 2$ Gy regimes are mostly applied; for ablative treatments a $6 \times 2$ Gy regime is common. 


\begin{tabular}{|c|c|c|c|c|c|c|c|c|c|}
\hline Fractionation (Gy) & $1 \times 2$ & $1 \times 8$ & $1 \times 9$ & $2 \times 2$ & $2 \times 2$ & $2 \times 4.5$ & $2 \times 5$ & $2 \times 6$ & $3 \times 3.3$ \\
\hline Fractions per day & 1 & 1 & 1 & 1 & 2 & 1 & 1 & 1 & 1 \\
\hline \# of institutions & 11 & 1 & 1 & 6 & 3 & 1 & 1 & 1 & 2 \\
\hline
\end{tabular}

\begin{tabular}{|c|c|c|c|c|c|c|c|}
\hline $3 \times 4$ & $4 \times 2$ & $5 \times 2$ & $5 \times 2$ & $6 \times 1.67$ & $6 \times 2$ & $6 \times 2$ & $8 \times 1.5$ \\
\hline 1 & 1 & $2 \times 2 \& 1$ & 1 & 2 & 1 & 2 & 1 \\
\hline 1 & 1 & 1 & 1 & 1 & 1 & 8 & 1 \\
\hline
\end{tabular}

Table 3.1 Reported dose-fractionation schemes for both ablative and non-ablative TBI-treatments in the Netherlands and Belgium.

Eleven out of 17 institutions have dedicated TBI teams to perform treatments. Twelve institutions schedule patients similar to other patient groups (regularly throughout the day). Two institutions schedule patients at the beginning, and three at the end of a day.

Fifteen out of 17 institutions have a designated backup machine. Two of these 15 machines are available at another (nearby) hospital such that a patient can be treated there on the same day. The other two institutions have no backup facility.

Eleven out of 15 institutions perform manual Monitor Unit calculations, occasionally using tabulated values. The other four perform dose calculations based on CT information in 2015, but this number increased to six in 2020. Several institutions expect to perform treatment planning based on CTinformation within the next few years, partly depending on replacements of their treatment planning system.

Most institutions (11) position the patient in a lateral decubitus position on an extended surface skin distance, varying from 3.7 to $5.5 \mathrm{~m}$. This requires a dedicated separate treatment couch. Other positioning methods are standing/leaning position (2) or allowing the patient to sit on a chair (3). Lateral positioning on the couch is commonly done using a vacuum mattress, chin to chest, drawn up knees, one arm along a side and the other arm supporting the head. Special attention has to be paid to extremity positioning for patient comfort and dose homogeneity.

Currently, 11 institutions use a 2-field technique with patients in lateral decubitus position. In all but one institution, the treatment couch is rotated 180 degrees in between fields; the remaining one 
alternates the patient's side position per treatment session. The others use 4 or 5 beams of which 4 institutions use field-in-field techniques in order to get a more homogeneous dose distribution. Beam energies vary from $6 \mathrm{MV}$ to $23 \mathrm{MV}$ (see Table 3.2).

The most frequently used treatment dose specification point is the midplane in the patient; either in a single point at e.g. the level of the umbilicus (10) or averaged over multiple midline points (3). Two institutions use full CT planning and therefore employ the body DVH for dose specification. Thirteen institutions reported their dose rate, being on average 20 cGy/min (range: 4-40 cGy/min).

\begin{tabular}{cc} 
Beam Energy & Number of institutes \\
\hline $6 \mathrm{MV}$ & 4 \\
$10 \mathrm{MV}$ & 5 \\
$15 \mathrm{MV}$ & 5 \\
$18 \mathrm{MV}$ & 2 \\
$23 \mathrm{MV}$ & 1
\end{tabular}

Table 3.2 Beam qualities used for TBI in all institutes

Critical organs, e.g. lungs, eyes, kidney and others are shielded from irradiation in various institutions, depending on the fractionation used. In general, for a total dose higher than 6 Gy shielding of organs at risk (e.g. lungs) may be considered. Lung shielding is performed in 11 out of 17 institutions, nine institutions shield lungs with individualized Cerrobend blocks, the other two with MLC. Eyes are shielded individually in one institution and standardized in four. Furthermore, one institution also blocks both kidneys individually, one institution uses Lucite plates to reduce the dose to the head if exceeding, e.g. $110 \%$ of the prescribed dose and finally one institution also shields ankles and knees in order to improve dose homogeneity. Two institutions do not use any shielding.

All but three institutions use Cerrobend blocks attached to a spoiler screen with double-sided tape; two institutions use MLC and one uses an in-house developed system with a screw/nut fixation. Block positioning is mostly verified by the naked eye, and four institutions use a dedicated mobile imager with a CCD detector to acquire MV-images (Theraview ${ }^{\mathrm{TM}}$, [84]). 
Responses to the pre-treatment QA questions were received from 17 institutions, of which 11 rely on the standard QA, i.e. no extra measurements are performed other than for non-TBI treatments. In addition, three institutions perform annual output measurements at TBI treatment distance. Patient QA is mostly performed by in-vivo dosimetry with either diodes, mosfets or similar tools (seven institutions) or TLD (two institutions) performed at least at the umbilicus and in few institutions also at other body positions such as head, lungs or abdomen. Tolerance levels were mentioned by institutions and vary from $3-10 \%$ of the prescribed dose (mostly 5\%).

Seven out of 17 institutions use AAPM report 17 [85] on TBI as a reference, 10 other institutions combine local experience in combination with various scientific papers.

For hygienic precaution measures, personnel in seven institutions wear masks and gloves in seven departments treatment couches are disinfected and personnel in four institutions use protective clothing. In two other institutions, standard hospital hygiene measures including hand disinfection are applied, and in four institutions, no special precautions are taken. The regimen depends on whether or not ablative fractionations are applied.

As part of their clinical implementation of TBI only four institutions have performed a risk analysis; three followed the HFMEA procedures $[86,87]$, the other provided no further information about the adopted method. 


\subsubsection{Results TSI}

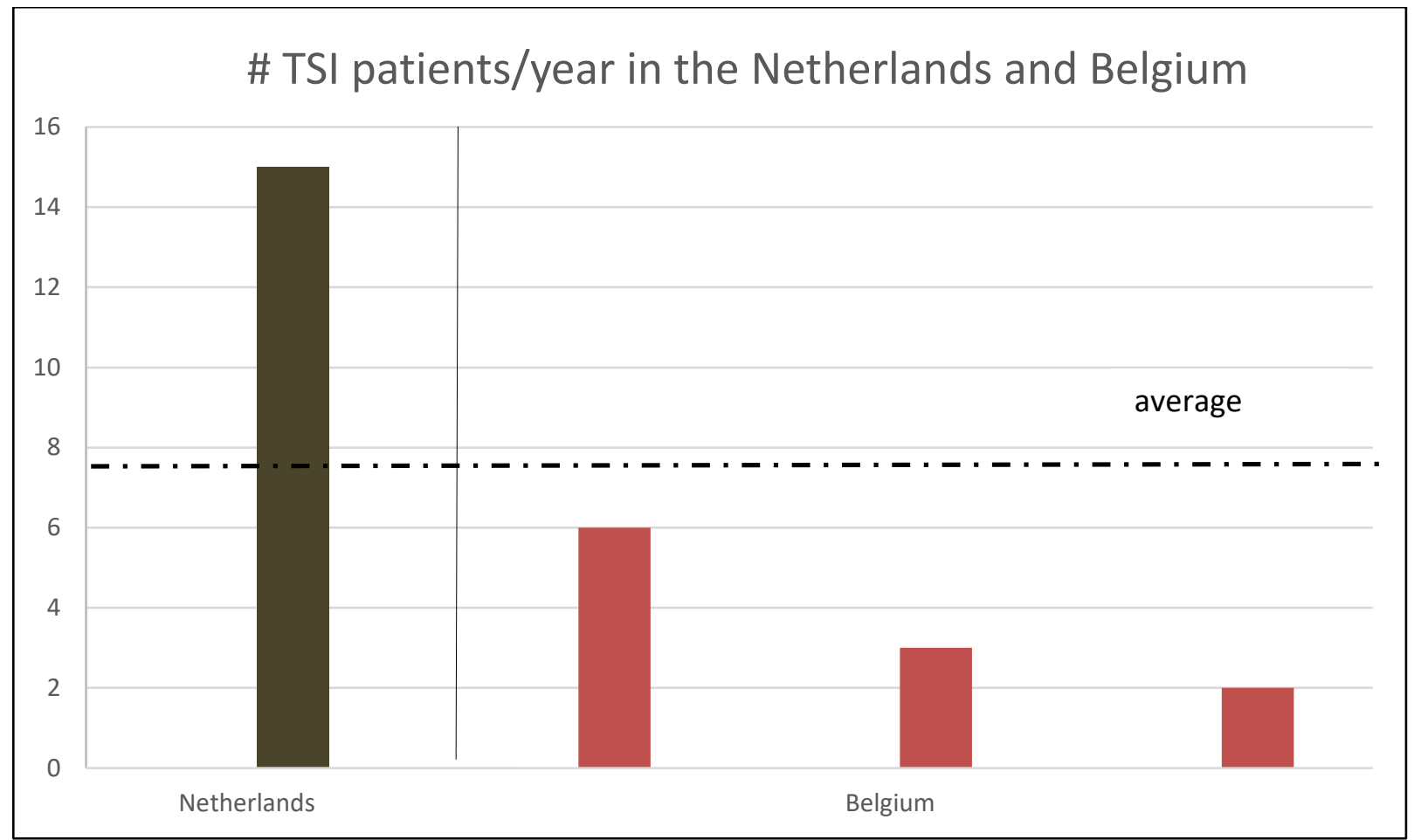

Figure 3.2. Number of TSI patients treated annually in the Netherlands and Belgium. Average value indicates average number of TSI patients annually for both countries.

TSI is mostly used to treat mycosis fungoides and occasionally skin lymphomas. The applied protocol is described in AAPM 23 (Stanford technique) [40]. The yearly average number of patients is 11 in Belgium and 15 in the Netherlands (see Figure 3.2). Fractionation schemes vary between $8 \times 1.5$ Gy on a daily basis and 7-10 × 3 Gy, every other day.

Three out of four institutions have a dedicated team for TSI treatments and the same three also have a backup machine on-site. All four institutions schedule their TSI patients on a regular basis, i.e., not at a specific time-slot.

All four institutions adopted the Stanford technique [40] treating the patient in standing position (two institutions) or lying on a stretcher (two institutions) in prone and supine position. Patient positioning is verified using visual markings and/or light field. The Stanford technique uses 6 positions and additional fields to crown, armpits, perineum, soles of the feet and in case of women the mammary fold if needed, additional treatment fields were given, based on in-vivo dosimetry performed at the first fraction. Electron energy varies from $4 \mathrm{MeV}$ to 6 or $9 \mathrm{MeV}$; the latter two in combination with a 
Lucite diffuser to increase surface dose and to prevent serious complications due to treatment of too large volumes.

Two institutions use simplified treatment planning with standard fields and tabulated MUs to account for patient geometry differences. One institution employs standard fields and MUs that have been verified as a standard class solution with no patient specific corrections. The fourth institution uses MUs that are based on solid water phantom measurements of the PDD and in-vivo dosimetry at the first fraction. In three institutions the determined MUs and machine parameters are manually entered into the record-and-verify system (R\&V); the fourth uses their R\&V-system to import standard plans from TPS. Three institutions rely on the standard machine QA whereas one institution periodically measures the dose in a phantom at treatment distance.

EBT-gafchromic film is used in two institutions for in-vivo dosimetry at about 10 anatomical locations, allowing for a maximum dose deviation of $10 \%$. A possible solution when exceeding the threshold is adjustment of the gaps between the separate fields. During treatment, the eyes are shielded using lead goggles in three institutions; the fourth institution does not use shielding (but also uses the lowest dose-scheme).

Two facilities have a backup machine on-site, 1 elsewhere and another has no backup. Note however, that TSI may be postponed since this treatment is not time-critical, in contrast to TBI. Dose calculations are table-based and performed manually and the maximum allowed dose inhomogeneity is between 10-20\%. All institutions verify patient positioning visually. No risk analysis was performed prior to clinical introduction of TSI techniques.

\subsection{Discussion/Conclusion}

Although TBI has been practiced in radiation therapy since the beginning of the previous century $[54,56]$ many variations exist among practices, even within countries as shown in various surveys $[57,61,62]$. Our survey in Belgium and the Netherlands is not only more detailed than previous surveys, (100\% response rate), but also includes TSI. Additional recommendations to existing guidelines [88] are made for centers that want to optimize their techniques or perhaps start with TBI and/or TSI.

Notwithstanding that Belgium and the Netherlands are small countries with not many institutions that practice TBI (19) or TSI (4), the applied techniques and procedures vary considerably concerning 
patient setup and shielding of critical organs, but also in terms of fractionation schemes and using dedicated teams or scheduling patients. Therefore, the variety in TBI treatment techniques over Europe as observed by Quast [57] and other more recent surveys, still holds for the current survey. Even though we did not distinguish pediatric TBI treatments, this group deserves special attention (TSI is not applied for this group in Belgium and the Netherlands). Hoeben et al. [89] describe a European survey and conclude that "there is a high uniformity in fractionation and in lung shielding" and that institutions are increasingly implementing new CT-based techniques. In addition, "a radiotherapy working group will be established to define international guidelines for pediatric TBI". In the Netherlands, all pediatric patients are referred to one dedicated institution (Figure 3.1, 25 patients/year). In Belgium, pediatric patients are treated in multiple institutions with low numbers.

In contrast to Japan where almost $20 \%$ of the 186 institutions use moving couch techniques $[60,61]$, Belgian and Dutch institutions applied large source-surface distances. In addition, the most common patient positioning in Japan is supine (more than 70\%), sometimes combined with AP-PA field configuration and a short source-surface distance.

Most large centers use a dose rate lower than $15 \mathrm{cGy} / \mathrm{min}$. Only 7 go up to $26 \mathrm{cGy} / \mathrm{min}$. In Japan, 80 percent of the centers shield lungs and almost $60 \%$ shield eye lenses (vs $65 \%$ and $31 \%$ respectively in our survey). In our survey lung shields consisted mainly of cerrobend blocks (almost $75 \%$ ). No specific time slots were scheduled but due to long treatment times TBI is performed during times when other radiotherapy types are not scheduled (46\%), or is limited to one fraction per day (7\%) or the number of TBI treatments is limited (20\%).

Nowadays a risk analysis is performed as (sometimes compulsory) part of clinical implementation of a treatment technique. Although not yet demanded by law, in the Netherlands a convenant 'Medical Technology' [90] has been developed in the medical technology community which prescribes risk analyses, included in periodically held national audits. However, this was not common or demanded in the past: techniques established long ago are generally not based on a risk analysis although occasionally a retrospective risk analysis is performed. An essential aspect of the risk analysis involves the level of expertise and experience (e.g. a dedicated team [63]) i.e. a minimum number of 5 annual treatments should be maintained in order to have a guaranteed expertise (see also Nelligan et al [63]). Therefore, a minimum of five annual treatments are recommended, preferably in a single center rather than distributed over multiple centers. 
If the time-interval between treatments is high (e.g. more than 3 months) then a dry run (chain test) is recommended.

One institution explicitly indicated that even though a backup machine is available on-site, recalculation of the monitor units to be delivered was needed due to different beam quality. A risk analysis would have shown it would be ideal to have identical machines, beams and treatment room sizes in order to prevent recalculations in an otherwise possible stressful situation. This is especially the case if a backup machine is available at a different (but nearby) institution as one responder indicated. For comparison, in Canada, all but two facilities had an identical backup machine [59].

The survey showed that 11 out of 17 institutions have a dedicated TBI team, and three out of four have a dedicated TSI team. Ideally, a dedicated team is deployed to perform TBI and/or TSI. From a resource perspective, such treatments could be most efficiently scheduled on pre-defined time-slots.

The efficiency of the scheduling also strongly depends on the workload of the team involved, especially if multiple fractions are given on the same day [63]. Other existing surveys did not mention data on time slots. In this survey 11 out of 17 institutions schedule TBI treatments on similarly as other nonTBI treatments, five schedule patients at the beginning or at the end of the working day. For TSI treatments, all four institutions schedule patients as a regular treatment.

Discussions of the survey results in the work group has already led to local adaptations of TBI techniques. For example, one institution treated patients for multiple decades with a dose rate of about $15 \mathrm{cGy} / \mathrm{min}$, but based on experiences by others and literature [91,92] they have recently increased the dose rate to its maximum (i.e., $40 \mathrm{cGy} / \mathrm{min}$ ). However, there is no clear consensus in the literature regarding this subject. Using a higher dose rate is an improvement from both an economical point of view and regarding patient comfort, because they may minimize the need to reposition patients during treatment. Recently imaging possibilities have become available to verify the positioning of patients $[84,93]$. Three participating institutes have purchased such a system and more are considering doing so. User experiences yet need to be published. Another institution adopted CTinformation for dose calculations.

Altogether, the survey has led to review and occasionally reconsiderations of the local treatment technique. Clear and more converging treatment techniques would also be beneficial for clinical outcome comparisons. 
A recent American survey among 101 institutions [66] showed that none of the institutions uses MLC in their TBI technique, and only $28 \%$ of the responders perform treatment planning based on CT information. Four out of 15 responders used CT information which is a similar ratio as in the USA. The recent survey update shows that the number of institutions that use CT has increased to six (i.e. $40 \%$ ).

Most centers in Belgium and the Netherlands practice lateral decubitus position, which increases homogeneity when using parallel-opposed pair treatment fields. However, not all patients found lying on their arm to be comfortable for the duration of the treatment [94]. Peters et al. [95]concluded that supine or combined supine-prone positioning is found most comfortable despite the fact that dose inhomogeneity will be increased. In contrast to this, Quast [38] recommends AP-PA TBI rather than bilateral TBI fields which causes low dose in mediastinum, ribs and arms, although the latter can reduce lung dose. However, modern imaging techniques prior to treatment, 3D treatment planning systems and dose verification systems are currently available to improve dose homogeneity irrespective of the treatment technique.

As discussed above clinical implementation of advanced technologies (e.g. CT, VMAT, and MLC) for TBI purposes goes relatively slow. Nevertheless, high advanced and complex technologies are studied in detail $[96,97]$. However, logistics, workflow and treatment times remain a challenge (e.g. Zoller, [80] as also was found by several institutions involved in this survey. On the other hand, high dose schemes might require more complex treatment techniques in order to spare OARs. However, this also depends on the expected clinical relevance. Finally, guidelines to practice TBI hopefully lead to more and more converging treatment techniques (e.g. Wong et al., Hoeben et al. and this report $[20,89]$ ) 


\section{Commissioning of TBI and TSI treatment}

\subsection{Introduction}

This chapter aims to describe all the necessary commissioning steps needed to implement a new workflow for either TBI or TSI safely. For the preparation and execution of TBI, different approaches exist that vary in concepts and details. First, we describe three different types of workflows that are used in the Benelux countries. Then, we describe the necessary commissioning steps, focusing on different aspects of delivering TBI safely.

For TSI one workflow is described in this chapter, if relevant, the TSI is described in separate paragraphs.

\subsection{TBI/TSI workflows; preparation and execution}

Below three possible workflows for TBI preparation and execution are discussed. The workflows are:

- Manual calculation workflow (4.2.1 for TBI and 4.2.2 for TSI)

- TPS workflow (4.2.3)

- $\quad$ The sweeping technique workflow (4.2.4)

Other workflows that have been reported, e.g. full VMAT/Tomotherapy workflows [97] and movingcouch workflows [98], are not covered in this report since these are not used presently in the Benelux countries.

The 10 steps in the 'manual calculation workflow' are followed by 10 equivalent steps in the 'TPS' and 'the sweeping technique' workflow. For TSI, only the manual workflow is discussed, since this is the only way in current practice.

\subsubsection{The manual calculation workflow: procedure without using a treatment planning system} (TBI)

In conventional workflows, no treatment planning systems (TPS) are used to facilitate TBI. An example of the treatment preparation procedure is as follows:

1. The patient is positioned in treatment position on a simulation device to determine the shape and thickness of the shielding. The image can be made either with X-rays, a CT or a megavoltage beam (6MV) using films (radiographic or radiochromic). 
2. From AP and lateral films the lung shapes are determined (by manual drawing on the shape on the film) and the thickness of the lung estimated by eye and pelvic callipers. Also, the use of a 'rule of thumb' to determine the effective thorax thickness (e.g. '1/2 $\times$ physical thorax thickness' or ' $1 / 3$ x lung thickness = equivalent normal tissue thickness') has been in circulation .

3. Patient thickness at different levels (including the level of the umbilicus) is determined using pelvic callipers.

4. Blocks are moulded from the drawings on the radiographical films using a manual styrofoam cutting device or using a digitiser/block milling device.

5. Monitor units are calculated using measured PDD/TMR/TPS tables (on paper or implemented in a spreadsheet) at TBI distance and the dose prescription at the level of the umbilicus or an average of umbilicus, hip and sternum.

6. An independent calculation of the MUs is done using a local procedure for clinical MU verification. Moreover, it is good to develop a feeling for the typical \# MU/Gy for your TBI technique, since this allows a quick check to avoid gross errors.

7. Block thickness is calculated using the required block transmission and a measured block transmission curve at TBI distance. The equivalent path length is calculated using a lung density table from literature. The transmission is calculated from the maximum lung dose which is obtained from the estimated equivalent lung thickness (see step 2 ) and patient thickness that is used for the dose prescription (see step 5).

8. Treatment is performed with the record \& verify system (R\&V); for some vendor/machine types using multiple beams of maximum 1000 MUs. Preferably using a template the beams are entered manually into the $R \& V$ system of the accelerator and the MU registration is also done manually (on- or offline).

9. Shielding position verification is done using portal (digital) films. This takes some additional time (5-10 minutes). The correction of the shielding position takes significant time, because a new portal (digital) film should be made after position correction. Repositioning of the shielding(s) (if necessary) can be done directly (online) or in a subsequent fraction (offline).

10. Every TBI treatment is verified using in-vivo detectors (e.g. TLD, MOSFET) with off-line readout especially when no R\&V system is used. 


\subsubsection{The manual calculation workflow (TSI)}

1. Total skin irradiation starts with a MU determination at extended SSD at Dmax. A reference chamber [99] for electron treatment is advised using an appropriate calibration factor (see 4.3.1). Due to the low frequency of the procedure, an absolute dosimetry in TSI conditions is advised prior to treatment. Alternatively, the ratio between dose/MU for TSI conditions at extended SSD, relative to the dose/MU at isocentric conditions can be determined. Since this allows checking TSI conditions during regular dosimetry.

2. If TSI is not tested regularly, functionality testing of the special features used for the treatments (e.g. high dose-rate mode and special electron applicators) and calibration of the in-vivo dosimeters is recommended prior to treatment.

3. Physical parameters (SSD, patient thickness, and weight) are of secondary order in the dose determination, allowing the use of a standard MU setting. Based on in-vivo dosimetry results the MU settings may be personalized after the first fraction.

4. MU adaptation for the individual treatment is linked to the obtained in-vivo results.

5. Treatment is performed with a record and verify system (R\&V), the beams are entered manually into the accelerator, preferably using a template. Exact labelling of the beams is necessary to avoid mistreatments.

\subsubsection{The TPS workflow: procedure including full integration of a TPS (TBI)}

There are no dedicated commercial treatment planning systems (TPS) for dose calculations for TBI treatments at extended distance. However, treatment planning systems are nowadays used frequently in the preparation of the TBI treatment.

Following the same 10 steps as above for the 'manual calculation workflow' the steps using a TPS for TBI at extended distance can be as follows:

1. The patient is positioned in treatment position including a vacuum mattress or similar positioning devices for scanning in a (big bore) CT simulator (to determine patient thickness, shape and thickness of the lung shielding); the CT images are transferred to the TPS.

2. In the TPS, the radiation oncologist or radiotherapy technologist draws the shape of the (lung) shielding on a DRR or the shape of the shielding is automatically generated from 3D delineated lung contours. In the latter case, the lung contour can be combined with, e.g. contours of the vertebral column to obtain the right shape. (Generally, a negative margin is applied to the lung 
to allow partly irradiation of the ribs; the vertebral column is somewhat extended to have a margin for residual movement). The body contour can be used for definition of the PTV, with a possible clipping of the surface with, e.g. $5 \mathrm{~mm}$, as there is always uncertainty about the skin dose calculation in the TPS.

3. Patient thickness is already included in the CT images (except the patient caudal part in case of limited scan length on the $\mathrm{CT}$ )

4. In case of physical shielding: the shape of shielding is sent to a block-milling device. As an alternative: dose reduction and homogenisation of the dose is performed using a beam sequence with forward- or inverse-planned IMRT beams (see Figure 4.1).
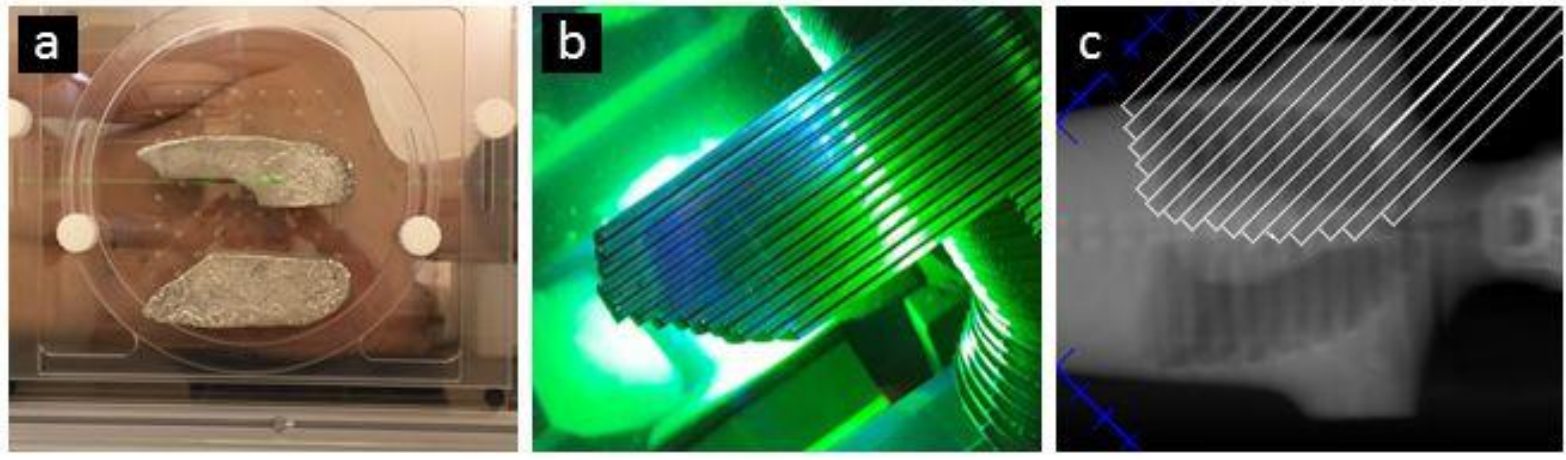

Figure 4.1 Dose reduction in TBI treatment. a) dose reduction of lung dose using Cerrobend blocks, b) example of MLC field to reduce lung dose and c) MLC field projected on DRR (courtesy of Radboud University Medical Centre, Nijmegen).

5. The dose is specified either in a dose prescription point (mid-line at the umbilicus or the averaged mid-line at the hip + umbilicus + sternum) or based on a DVH and monitor units. The maximum and average doses in the lung and other OARs are calculated by the TPS using a 3D calculation model. The beam model used is either:

- the standard beam model, which is also verified for TBI treatments;

- or a special TBI beam model optimized for, e.g. output or off-axis ratio ${ }^{1}$, in case the standard beam model deviates too much when calculated using the clinically used beam model (In general the deviations are not very large, see 4.3.1g).

Dose inhomogeneity can be corrected using field-in-field technique of extra beams (or segments).

6. An independent calculation of the MUs is done. Ideally, this is an automated procedure for clinical MU verification (using DICOM objects).

\footnotetext{
${ }^{1}$ See section: 4.3.1 Absolute beam calibration in TBI/TSI-reference setup (TBI/TSI)
} 
7. Only when physical shielding is used (see step 4): thickness calculation of the shielding is done using a dose specification in the OAR and the DVH value (maximum or average) of the unshielded OAR. Note that using a Mean Lung Dose (MLD) would define the lung dose more robustly. In case the TPS is capable of calculating the dose with shielding at TBI distance accurately enough, the shielding can be included in the dose calculation. In case the TPS is unsuitable for that purpose, the thickness of the shielding(s) should be calculated separately using the required transmission and a tabulated transmission curve at TBI distance (see 4.3.4).

8. Treatment is performed using a DICOM transfer of the beam configuration from the TPS to the R\&V system.

9. The position of the shielding is verified using a digital portal imager or self-developing (radiochromic) film with the DRR including the shielding as reference image. Correction of the position of the shielding or of the patient (in case shielding is done using the MLC) can be done online using a portal imager $[84,100]$. With films the correction can be done on- or offline.

10. In-vivo dosimetry can be used for the first 10 patients as check of the 'class solution 'or for the verification of every patient to check for consequences of changes in setup/anatomy. See 4.3.6.

\subsubsection{The sweeping technique workflow (TBI)}

Using a sweeping technique with fixed (maximum) field size for TBI treatments is relatively new. This technique also involves many 'manual' steps. This technique is interesting for institutions where a large SSD (e.g. $>200 \mathrm{~cm}$ ) is not feasible. The first publication for TBI of this technique (but with a fixed dose rate) is from Pla et al [101]. More recent literature (including variable dose rate) is from Zhuang et al [96], Jahnke et al [70] using a $10 \times 40 \mathrm{~cm}^{2}$ field size and Polednik et al. [102] using a $40 \times 40 \mathrm{~cm}^{2}$ field size.

1. Two CT-Scans are performed with the patient in both treatment positions (supine and prone). Marks are placed on the patient to locate the umbilicus

2. The dosimetrist (or radiation oncologist) draws the shape of the lungs and measures the distance between the surface of the patient and the couch at the umbilicus for both CT-scans. Patient thickness is considered as the average thickness, at umbilical, of the two CT-scans.

3. The lung contours projection on the DRR is used to shape the block shielding. Take care to correct the shape of the block to maximize the dose coverage (for ribs, spinal cord, liver and collarbone). The shielding thickness is based on attenuation measurement (on an 
anthropomorphic phantom in TBI-conditions) and the dose specification at the OAR. For example to reduce the lung dose to $80 \%$ of the prescription the primary fluence has to be reduced to $60-70 \%[38]$.

4. The dose is specified at the mid-line of the umbilicus and the total MU is based on patient thickness and measurement of the dose at middle of a phantom with different thicknesses in TBI treatment configuration.

5. A generic arc is weighted by changing the total MUs by the MUs calculated in step 4 in the record and verify system. The generic arc was created to achieve a homogenous dose in the middle of a phantom (with thickness of $19 \mathrm{~cm}$ ) with a control point every 5 degrees. For more details about the generic arc see Polednik et al [102], using a $40 \times 40 \mathrm{~cm}^{2}$ field size.

6. Pre-treatment verification is done for every arc using diode detectors and an ion chamber (mainly to check that the arc was correctly weighted before the actual treatment). The ion chamber is used to check the calibration factor of the diode for in-vivo dosimetry.

7. The patient is placed on a dedicated moveable couch. The umbilicus of the patient is positioned at the crossing of sagittal and transverse laser. No additional laser is used. A beam spoiler ( $1 \mathrm{~cm}$ of Plexiglas placed $30 \mathrm{~cm}$ above the surface of the couch) is used to maximize the skin coverage and the lungs shielding are directly place on patient skin.

8. The lung shielding is directly placed on the patient skin. The block position verification is done using a digital film (phosphorus plate, MV compatible).

9. The first arc is realised in supine position and the second in prone, both aligned on umbilicus (using the mark from the simulation).

10. Every TBI treatment is verified using detectors ( $5 \%$ of difference tolerance), placed both on the beam spoiler and on the patient skin for all position (HFS and HFP). Dose corrections are done offline in the following fraction(s). See section 4.2 .2 for correction strategies. 


\subsection{Commissioning of TBI/TSI technique}

Before TBI/TSI can be performed clinically, the following subjects should be addressed.

1. Absolute beam calibration in TBI/TSI-reference setup (TBI/TSI)

2. Beam Flatness (beam profiles i.e. off-axis ratios) at TBI/TSI-distance (TBI/TSI)

3. $\mathrm{PDDs}$ at TBI/TSI-distance (TBI/TSI)

4. In-vivo dosimetry systems (TBI/TSI)

5. Dry runs/end-to-end test and in-vivo dosimetry (TBI/TSI)

6. Extra positioning lasers (TBI/TSI)

7. Shielding transmission (TBI-only)

8. Extra commissioning steps for TPS workflow (TBI-only)

9. Extra measurements for sweeping techniques (TBI-only)

10. Other commissioning items (TBI/TSI)

11. Cardiac Implantable Electronic Device (CIED) and other electronic devices ((TBI/TSI)

More details about these items are described below.

\subsubsection{Absolute beam calibration in TBI/TSI-reference setup (TBI/TSI)}

The reference dose rate should be measured at the clinically used TBI/TSI-distance in the TBI/TSI-reference setup.

Details of the TBI/TSI-reference setups:

TBI:

In this large field geometry (maximum field size $40 \times 40 \mathrm{~cm}^{2}$ at isocentre), the output could be measured at an effective depth of $10 \mathrm{~cm}$ in a phantom of at least $30 \times 30 \times 20$ $\mathrm{cm}^{3}$ (20 $\mathrm{cm}$ in beam direction). It should be either a water phantom or a Solid Water phantom. The output in this TBI-reference setup could be related to the output in a standard machine calibration procedure $(S S D$ or $S A D=100 \mathrm{~cm})$.

Remark:

in case TBI treatment planning is performed using a TPS, the size of the phantom can also be different (e.g. smaller). The same phantom size should then be used in the TPS to verify the reference output.

TSI:

The minimum phantom size can be smaller: about $10 \times 10 \times 10 \mathrm{~cm}^{3}$ and the output should be measured at depth of maximum dose. It should be either a water phantom or a 
Solid Water phantom. The output in this TSI-reference setup should also be related to the output in a standard machine calibration procedure (SSD or SAD $=100 \mathrm{~cm})$. The chamber must be validated for the dose rate used in TSI treatment settings. For the absolute dosimetry special energy correction factors must be applied while at extended SSD energy changes are expected due to electron air interactions and due to the large field size versus the field size of the reference conditions.

Special attention should be given to:

a. Chamber properties

- $\quad$ TBI: Measured ionization chamber current may be of the order of the leakage current especially if low dose rates are used. So do not use too small ionization chambers (a 'Farmer -type' ionization chamber with volume of 0.6 $\mathrm{cm}^{3}$ should be sufficient in most cases).

- $\quad$ TSI: NACP or Roos chamber [99].

- Influence of the ionization chamber cable irradiation: do not place cable extension connectors inside the radiation beam.

- In general, it is advised to use the same type of ionization chamber which is used for reference dosimetry [99].

- If TSI is performed in a high dose rate mode the recombination correction has to be determined and corrected for [99].

b. Phantom dimensions

For TBI the finite dimensions of the phantom can play a role in the ionization measured. See AAPM Report 17, paragraph 3.2 [85] for more details and correction methods. Corrections are usually small (1 - $2 \%)$ and can in many cases be neglected.

c. Large number of MUs

Due to the large SSDs in TBI/TSI irradiations the number of MUs given is usually large (>1000), compared to standard clinical treatments with static beams. In general the stability of the treatment machine (output) is not a problem for this amount of MUs, but it should be verified when the treatment machine is commissioned. It is recommended to do the absolute dose calibration using MUs in the clinically used range of MUs. Moreover, it should be checked what happens beyond $1000 \mathrm{MUs}$. 
d. Beam spoiler screens (TBI) and beam scatterer-energy degraders (TSI)

TBI:

in case a beam spoiler screen / build-up screen is used to increase the skin dose of megavoltage beams these screens should be included in the absolute beam calibration. To avoid double compensation for the screen, it should always be clear if the screen is included in the absolute beam calibration or not. When a TPS is used in the TBI procedure, this has to be checked explicitly, with respect to the attenuation and the build-up effect.

TSI:

in case $4 \mathrm{MeV}$ is not available a beam scatterer-energy degrader ('decelerator') is used to reduce the energy. The beam scatterer also improves dose uniformity, see AAPM Report 23 [44]. This scatterer should also be included in the absolute beam calibration.

e. TSI-only: Field size for large electron beams Depending on the vendor/machine type large electron beams $\left(40 \times 40 \mathrm{~cm}^{2}\right)$ can sometimes only be given using a dedicated applicator (e.g. called 'null applicator'). This applicator has to be accepted and commissioned explicitly in case it is not a part of the standard applicators used for other clinical electron treatments.

f. Different treatment rooms

Generally, treatment can be performed in different treatment rooms, hence absolute beam calibration should be checked for the machines in all used treatment rooms. For matched treatment machines, the absolute dose calibration for the TBI/TSI-reference setup is expected to be equal within $\pm 2 \%$.

g. TPS-calculated output

When a TPS is used for dose calculation, the measured output in reference conditions should match the calculated output within $\pm 3 \%$, (see NCS report 15 , table A.4.1.1: complex geometry [103]). In general, a dedicated TBI beam model for use at TBI conditions is not necessary because the standard beam model is accurate enough in a modern TPS with a collapsed cone beam model (or equivalent). In case a dedicated TBI beam model is commissioned in the 
TPS to compensate for differences in output and flatness, make sure the beam models for standard SSD and TBI cannot be mixed by users.

\subsubsection{Beam Flatness (beam profiles i.e. off-axis ratios) at TBI/TSI-distance (TBI/TSI)}

Because flattening filters (TBI) or scattering foils (TSI) are designed to obtain a flattened beam at a specific depth with SSD/SAD= $95-100 \mathrm{~cm}$, the beam profile for a large field at TBI/TSI-distance should be verified, for TBI at least along the cranio-caudal axis of the patient (e.g. along the diagonal of the maximum field size).

TBI: This could be done at low spatial resolution (e.g. every $10 \mathrm{~cm}$ ) with an ionisation chamber at different positions in the beam using a small (water) phantom, e.g. at a depth of $10 \mathrm{~cm}$, or a large build-up cap (diameter $10 \mathrm{~cm}$ or larger). Increase the spatial resolution, e.g. every $1 \mathrm{~cm}$ near the edge of the field. Alternatively, measurements can be performed at higher spatial resolution using a large water phantom with a scanning ionisation chamber, and by positioning the large water phantom at different positions along the profile (taking care to scan not too close to the edge of the phantom, e.g. > $10 \mathrm{~cm})$. In this case care should be taken while "stitching" the different scans/measurements (allow some overlap between two scans). A linear or 2D array mounted in solid water phantoms at a relevant depth of, e.g. $d_{\max }, 5$ or $10 \mathrm{~cm}$ can also be used to measure profiles at depth. Choose the appropriate spatial resolution so that the $90 \%-90 \%$ profile distance can be identified in the cranio- caudal direction (for example if a collimator rotation of $45^{\circ}$ is used). This profile is very important for this application and should be regarded with due attention.

No TPS used:

If no TPS is used for dose calculation the maximum variation in off axis ratios found at a specific depth (e.g. 5 or $10 \mathrm{~cm}$ ) related to the value at the beam axis should not exceed the clinical requirement (e.g. $\pm 10 \%$ ). In case the variation is too much, special flattening filters can be used or a PMMA-compensator (which can be mounted on the beam spoiler screens or on the tray).

TPS used:

In case a TPS is used for treatment planning, the measured profile should match the profile calculated by the TPS in the same situation within e.g. $\pm 3 \%$, see NCS 
report 15, table A.4.1.1: complex geometry [103] or less strict depending on the clinical requirement.

TSI: The beam profile for a large field should be measured using a diode or a thin parallel plate ionisation chamber along the field profile at discrete positions $(e . g$. every $10 \mathrm{~cm}$, and smaller steps in the penumbra region $(<10 \%$ difference between consecutive measurement points) or by using linear or 2D chamber arrays at depth of maximum dose. Often superposition of oblique fields is used to obtain a homogeneous dose distribution implying the verification of the depth of maximum dose of an individual field versus the depth of maximum dose of the total dose distribution. Profiles should be validated with clinical setups, so superposition of fields must be validated before treatment (see Figure 4.2a, Figure 4.3 and Figure 4.4). This can be done using an anthropomorphic phantom wrapped around with film and build-up material or a film placed at an axial slice (see Figure 4.6). Electron diodes can be used if their energy dependence is checked. For some electron diodes this energy dependence is reported and a cross calibration is advised with a reference detector for standard electron treatments versus TSI treatment conditions.

The maximum variation in off-axis ratios found at dose maximum related to the value at the beam axis should not exceed the clinical requirement (e.g. $\pm 10 \%)$. In case the variation is too much, special scattering foil can be used. 

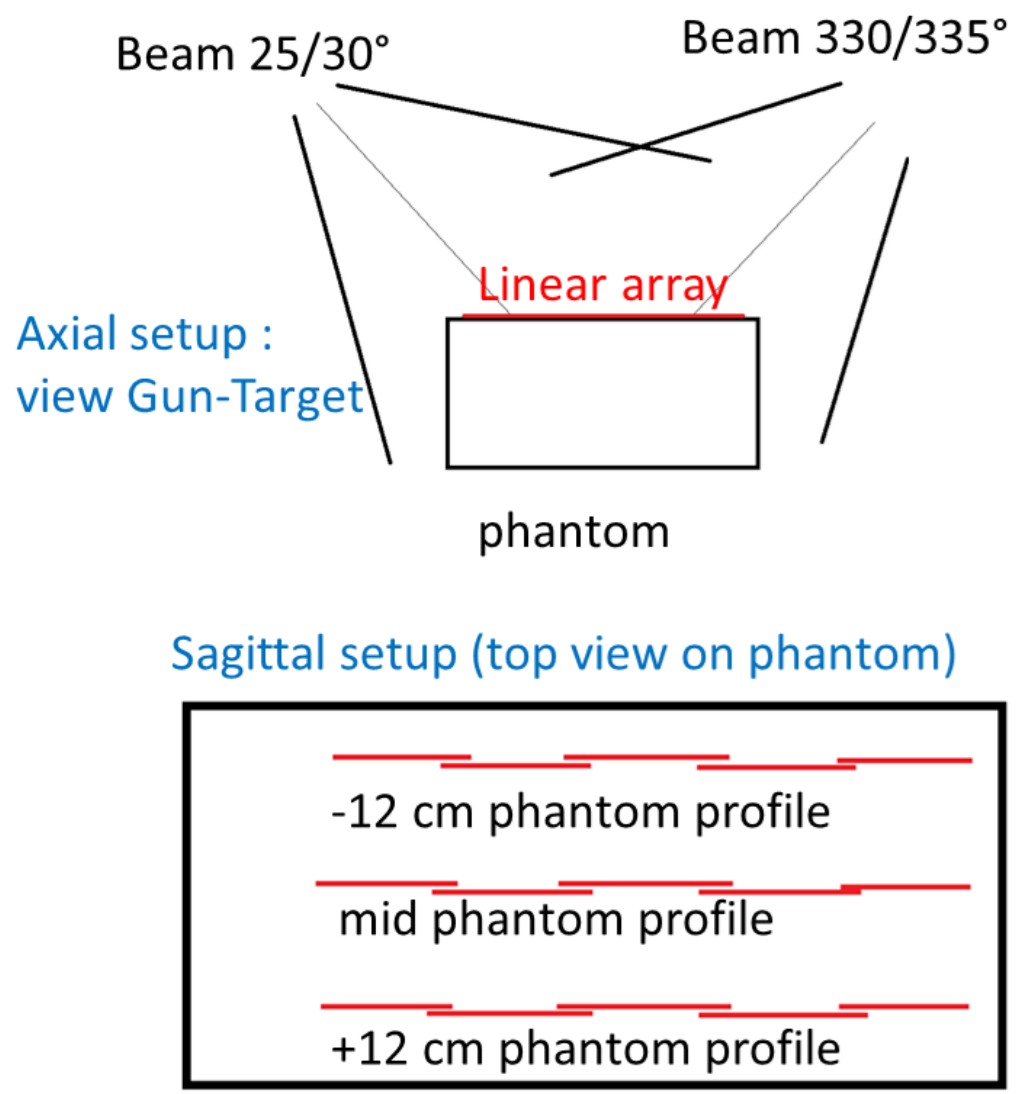

Figure 4.2a Total skin external beam radiotherapy uses superposition of oblique fields. This technique results in more homogenous dose distribution along the axial slices and increases the skin dose versus the dose at Dmax. While for instance the $D_{\max }$ is situated around $8 \mathrm{~mm}$ for a $4 \mathrm{MeV}$ beam, oblique incidence reduces this maximum depth to $4-5 \mathrm{~mm}$ and the difference in dose at the surface versus at $4 \mathrm{~mm}$ decreases. The red lines $(5 \mathrm{x})$ at 3 position in the phantom $(-12 \mathrm{~cm}$, mid-plane and $+12 \mathrm{~cm})$ represents the 5 linear array positions. The measurement results are displayed in Figure $\mathbf{4 . 3}$ (courtesy of Universitair Ziekenhuis Gent). 


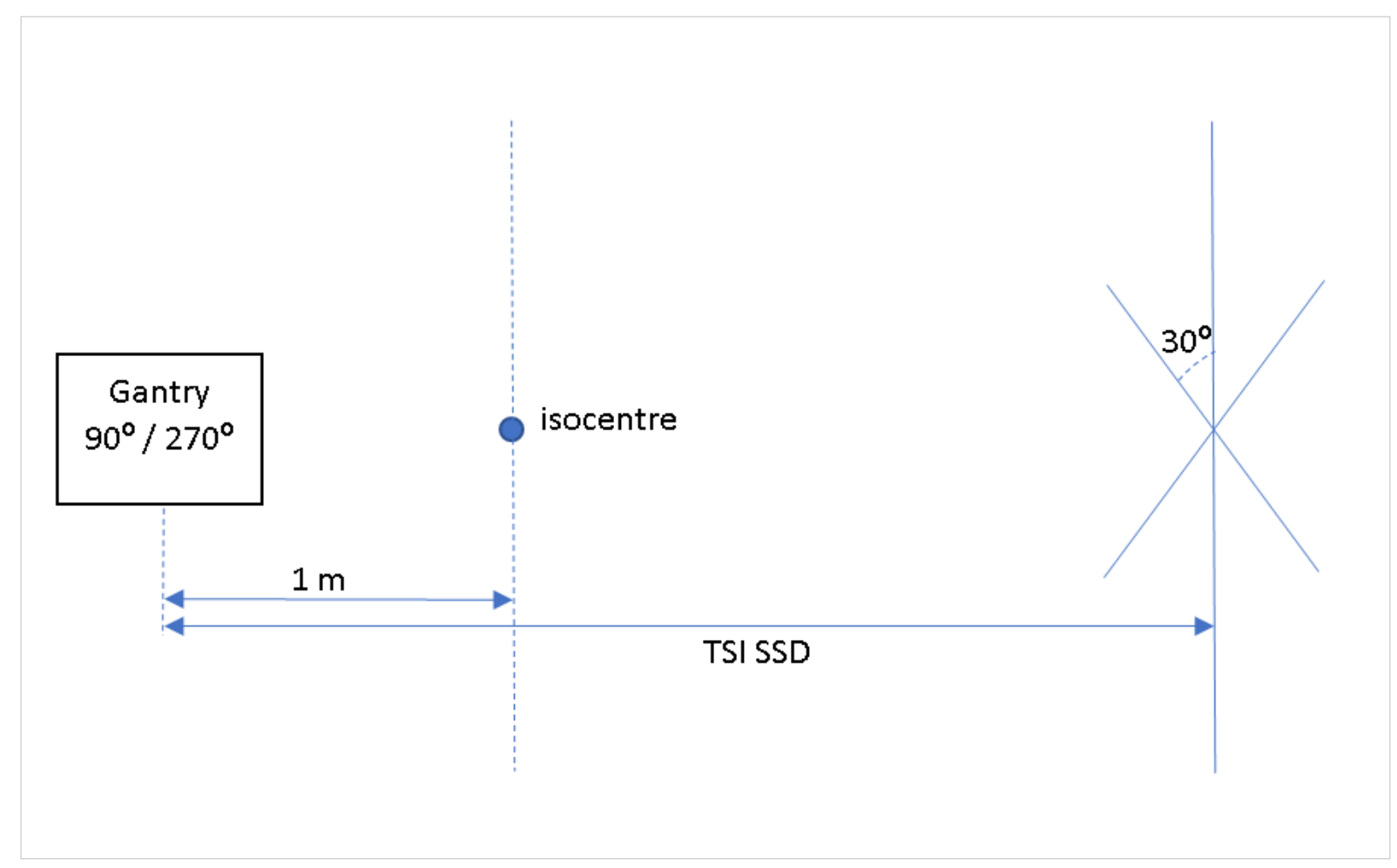

Figure 4.2b Floor pattern for TSI

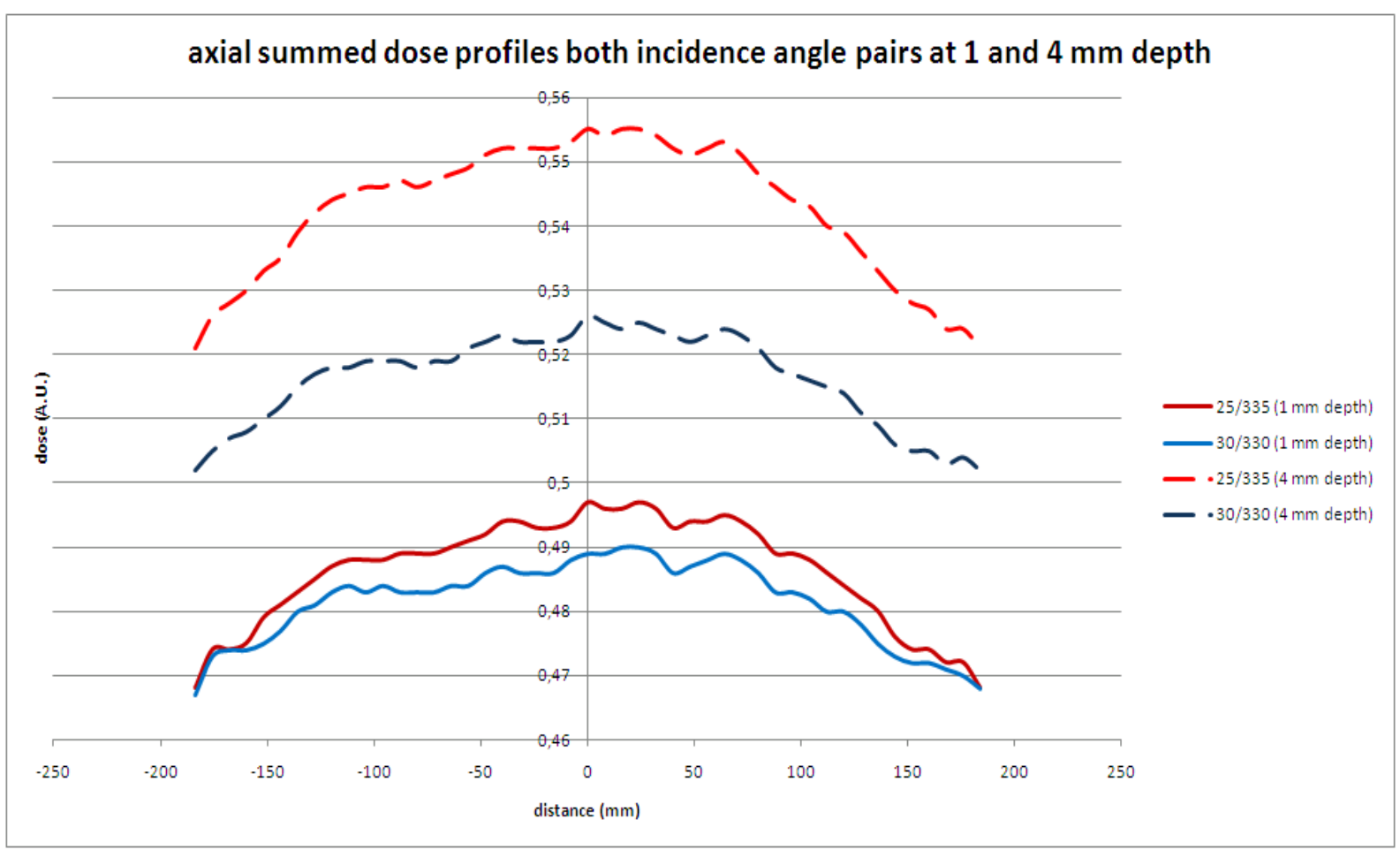

Figure 4.3 $\mathrm{A} 60^{\circ}$ difference in incident beam angles results in an overall more homogenous beam at $4 \mathrm{~mm}$ depth and at skin level, compared to the $50^{\circ}$ difference and reduces the dose difference between skin dose and maximum dose at $4 \mathrm{~mm}$ depth (courtesy by Universitair Ziekenhuis Gent). 
Profile GT direction $4 \mathrm{MeV} 40 \times 40 \mathrm{~cm}^{2}$ at $171 \mathrm{~cm}$ Oblique Incidence

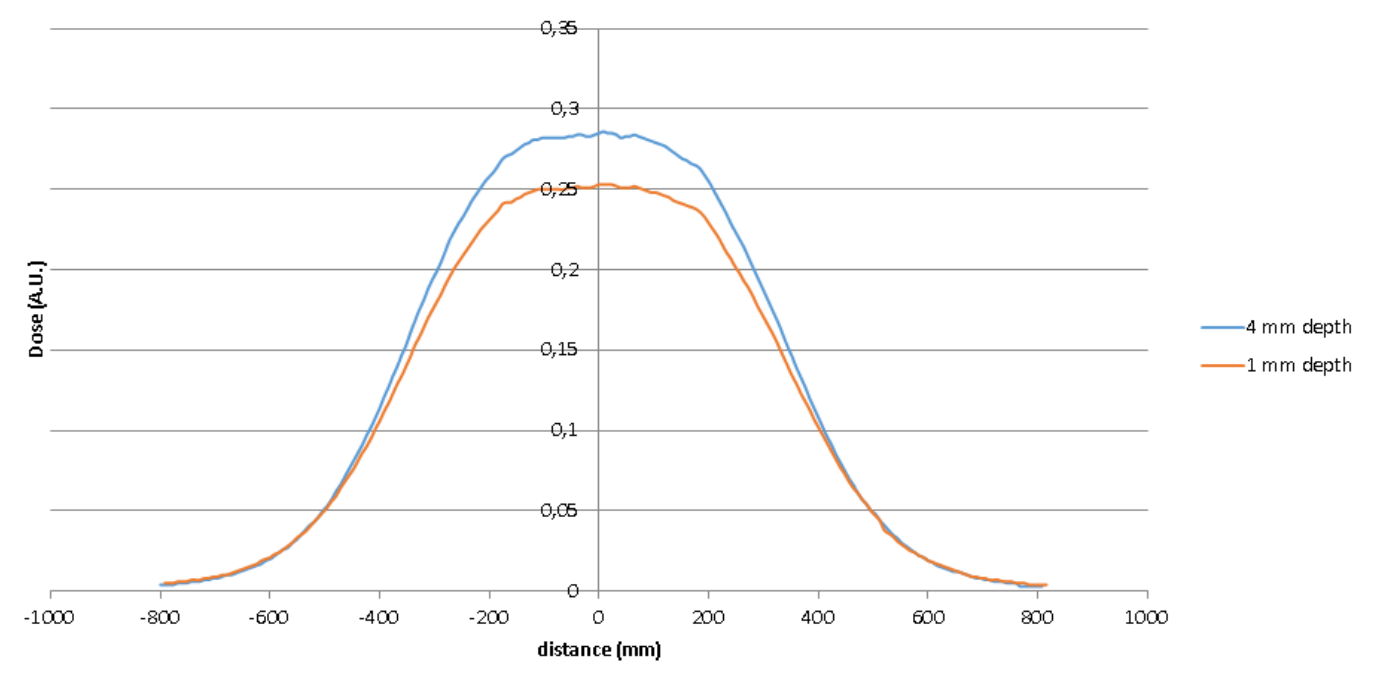

Figure 4.4 Sagittal profile long the mid-line of a phantom $(12 \mathrm{~cm}$ off axis for left beam and $-12 \mathrm{~cm}$ off axis for right beam, see sagittal setup in Figure 4.2a). The large penumbra is due to the lack of trimmers.

The skin dose is $90 \%$ of the dose maximum. Since no trimmers are used for total skin electron beams and the SSD is $150-350 \mathrm{~cm}$ a very shallow penumbra is characterizing the beam profiles. The light field equals $68 \mathrm{~cm}$ (corresponding with the FWHM). The therapeutically useful part of the radiation field (85-85\% isodose distance) however, is only about $50 \mathrm{~cm}$. Because of the large penumbra patient setup, using light field agreement should be avoided (courtesy by Universitair Ziekenhuis Gent).

\subsubsection{PDDs at TBI/TSI-distance (TBI/TSI)}

PDDs should be measured at the clinically used TBI/TSI-distance. It should be kept in mind that due to energy spectrum changes, off-axis PDDs can differ from PDDs at the beam axis (especially in the corners of a collimator-rotated field), but usually the difference is clinically irrelevant e.g. $< \pm 3 \%$. However, during patient treatment, care should be taken to keep the patient within the $90 \%-90 \%$ values of the profile.

Furthermore, if shielding is used, the PDD changes behind the shielding because of low-energy scatter from the irradiated part of the spoiler screen outside the shielded area, and (for low energy beams) due to beam hardening. Measurements could be performed to characterize these effects.

If compensators are used their influence on the energy distribution of the transmitted beam should be evaluated by comparing non-compensated PDDs with compensated PDDs. However, this influence is expected to be a minor issue in case of TBI. 
TBI:

For measurement in a water phantom in a horizontal beam the effective thickness of the wall of the water phantom should be included in the setup. When the thickness of the wall of the water phantom is identical to or smaller than the thickness of the beam spoiler, no separate measurements in the build-up regions are necessary. Cylindrical chambers are suitable for PDD measurements.

For measurement in the build-up region of the beam, parallel plate ionisation chambers in a plastic (e.g. solid water) phantom should be used. These measurements should be performed including the beam spoiler screen / build-up screens in case they are used clinically. Rawlinson et al and Velkley et al $[104,105]$ provide correction factors for the use of a parallel plate ionisation chamber instead of an extrapolation chamber to obtain the surface dose.

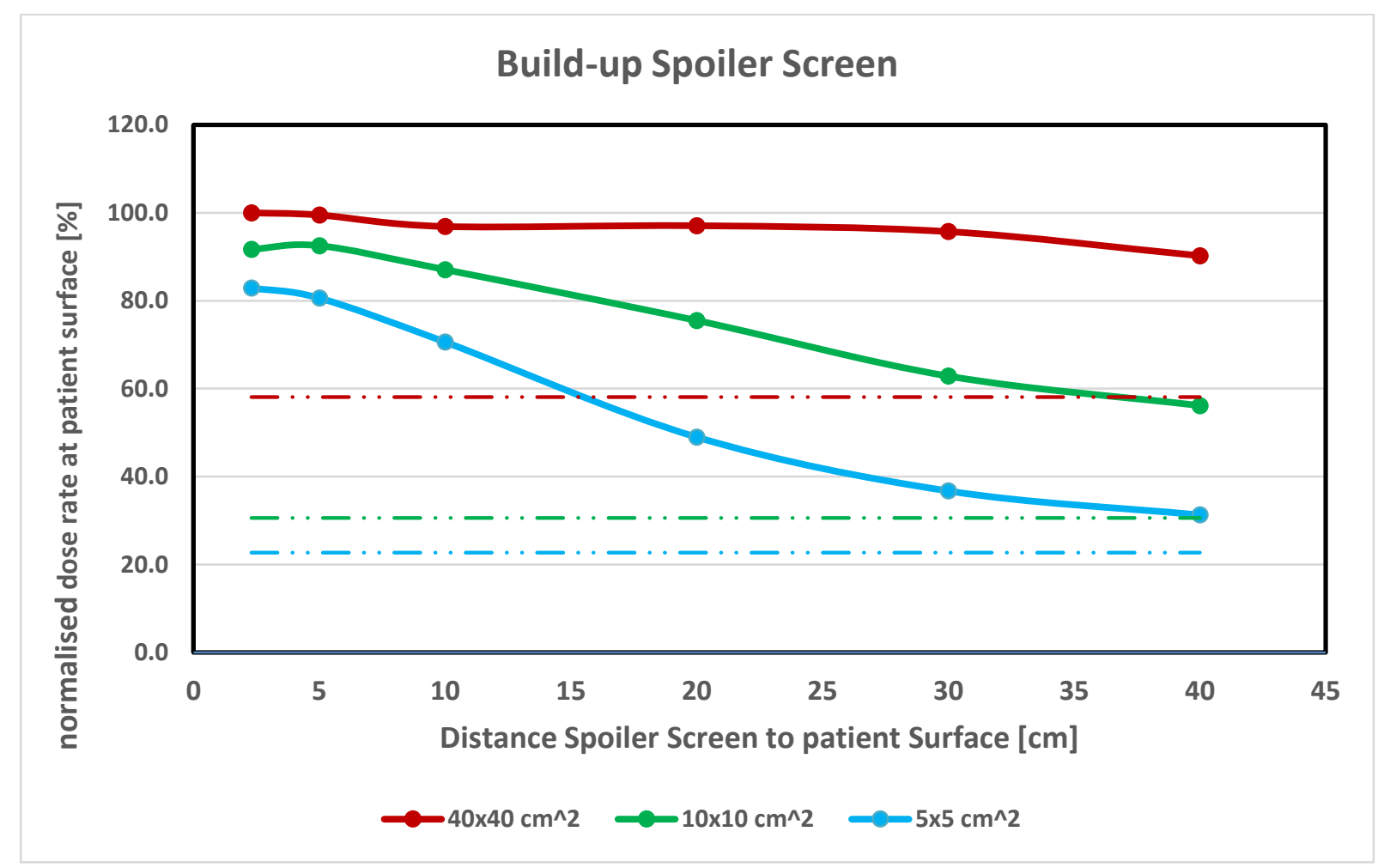

Figure 4.5 Build-up skin dose as measured on the beam axis for field sizes of 40x40, 10x10 and $5 \times 5 \mathrm{~cm}^{2}$ with a mosfet detector on the surface of a PMMA phantom at a source-surface distance of $370 \mathrm{~cm}$ using $10 \mathrm{MV}$ (measurement errors 4\%). The $1.5 \mathrm{~cm}$ PMMA spoiler screen is placed at 2.3, 5, 10, 20, 30 and $40 \mathrm{~cm}$ of the surface. The dose is normalised to the maximum dose measured, with the largest field $\left(40 \times 40 \mathrm{~cm}^{2}\right)$ at a distance of $2.3 \mathrm{~cm}$ from screen to surface. Dashed lines indicate dose with no screen in the beam (no build up) for 40x40, $10 \times 10$ and $5 \times 5 \mathrm{~cm}^{2}$. Measured by Maastro, the Netherlands.

The effect of the use of a spoiler screen is shown in Figure 4.5. It shows that the spoiler screen actually improves the skin dose; however, the effect depends on the field size and the distance from screen to patient. The additional skin dose is a result if the secondary electrons generated 
in the screen, which contribute less at a larger distance (loss due to scatter) or at smaller field sizes (less electrons generated). In general, a $40 \times 40 \mathrm{~cm}^{2}$ field size will provide a stable build-up effect over up to $30 \mathrm{~cm}$. If a field-in-field technique is used, the skin dose can be compromised because of the possible small segments. Since most TPS do not calculate the surface dose reliably, it is advised to measure the skin dose for verification.

For hand / tabulated dose calculation the PDDs can be converted to TMR or TPR [106].

In case a TPS is used, it is sufficient to verify calculations with phantom measurements at several clinically used depths. In the calculations, conditions should be similar to the measurements.

TSI:

Because of the extended SSD used during TSI, the relative influence of the inverse square law decreases. Besides, due to interactions with air, the energy spectrum changes and, therefore, PDDs for TSI differ from PDDs at standard SSDs. Since superposition of fields is used, oblique incidence for some beams is used to homogenise the dose distribution influencing the PDD as well. However, the exact PDD is not critical for TSI. The treatment is superficial and therefore only the combined output of all clinically applied fields, should be measured at a depth of 3 to $5 \mathrm{~mm}$. NCS18 [99] recommends the use of a NACP or Roos chamber for the output measurement.

\subsubsection{In-vivo dosimetry systems (TBI/TSI)}

In case in-vivo dosimetry systems (e.g. diodes, MOSFETs, TLDs, radiochromic films) are used to register and verify the dose during TBI radiation, these systems should be commissioned for TBI/TSI conditions (large SSD, field sizes, fraction dose, low dose rate, with spoiler screen). In case diodes are used, calibration should be done using a phantom in a TBI/TSI-setup. The relation between entrance/exit/mid-plane dose should be established and verified in a phantom measurement for TBI conditions (see, e.g. Bloemen et al. [94]). This procedure should also include the use of the beam spoilers.

Any relevant correction factor should be applied to correct the readings taking into account e.g. energy dependence, incident beam angle dependence and field size dependence. In order to ensure correct readings these dependences should be validated on a regular basis against the reference dosimetry system placed mid-line [63]. 
Please note that when performing TBI measurements behind shielding on the entrance side, there is a significant contribution of dose from the unshielded part if a beam spoiler is used. The contribution of the unshielded part of the beam spoiler consists of low-energy scattered radiation. Separate measurements could be performed to characterize this effect. During in-vivo measurements, this effect can be reduced by adding, e.g. $1 \mathrm{~cm}$ of bolus material (be aware that this can cause an increased skin dose).

For TSI, reference dosimeters for electron beam calibrations are to be used to determine calibration factors.

\subsubsection{Dry runs/end-to-end test and in-vivo dosimetry (TBI/TSI)}

To verify the commissioning of a TBI /TSI procedure a dry run (also called 'end-to-end test' or 'dummy run') should be performed. In this dry run, the personnel involved in the preparation and treatment procedure should perform all steps. Preferably, an anthropomorphic phantom (e.g. the Alderson Radiation Therapy phantom) should be used in which dosimeters (e.g. TLDs, MOSFETs, radiochromic films) can be placed. The whole treatment procedure including the verification (and for TBI: possible correction of the positioning of the shielding(s)) should be performed (also the use of the remotecontrol patient positioning in case this is utilised). The dose given to the phantom can be evaluated using the TLD/MOSFET entrance/exit readings or radiochromic films/TLD dosimeters positioned inside the phantom (TBI) or near the skin of the phantom (TSI).

TBI

The transmission of the individual shielding is measured for the first patients (e.g. 10) to verify the correct shielding transmission.

In case a TPS is used, patient plans could be transferred to scans of a dosimetry phantom for more accurate QA measurements, e.g. using a Farmer-type chamber in a standard dosimetry phantom. It is also advisable to determine the MU range for TBI to detect gross errors in dose calculation early in the planning process. Besides, the MU check will do a more thorough check. 
TSI

Measurements can be performed with films inside a RANDO phantom that were cut, to follow the contour of the phantom, as depicted in Figure 4.6) Also films underneath a $5 \mathrm{~mm}$ bolus wrapped around the RANDO can help to verify the correct field functions.

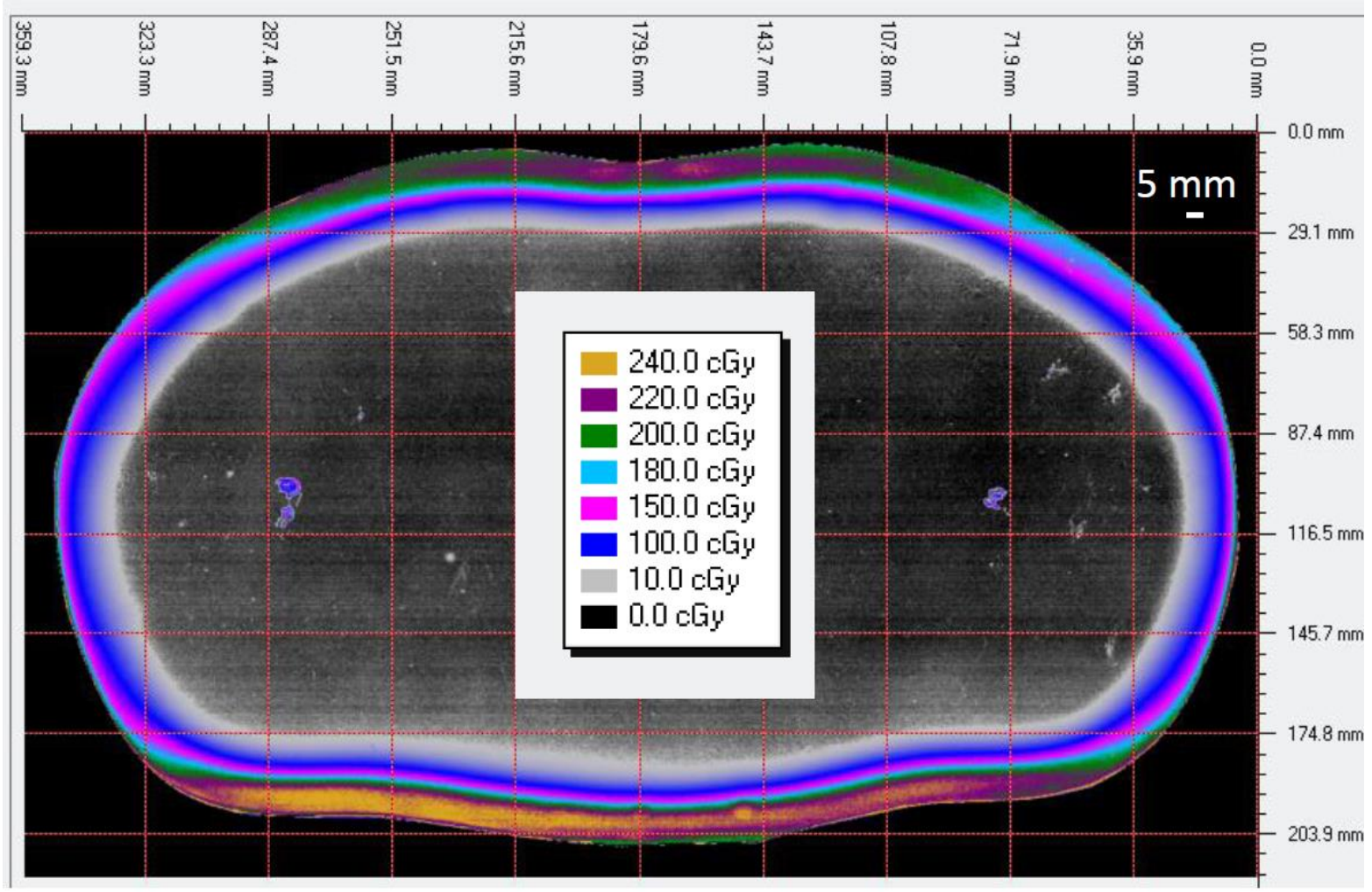

Figure 4.6 TSI-film dosimetry in the RANDO phantom (courtesy by University Medical Center, Leiden)

In-vivo dosimetry

It is also advised to do in-vivo dosimetry at least for the first 10 patient as extra quality check of the whole TBI / TSI procedure. The entrance/exit dose at the prescription plane could be measured using TLD/MOSFETS and converted to mid-plane dose with a calibration curve.

\subsubsection{Extra positioning lasers (TBI/TSI)}

$\mathrm{TB} \mid \& \mathrm{TSI}$

For extended-SSD techniques, extra positioning laser equipment should be installed to assist patient positioning for TBI / TSI treatment at the treatment SSD. The QA of these laser(s) should be included in the quality assurance of the treatment machines. The accuracy of the vertical longitudinal laser(s) 
at extended SSD is not that critical ( $\pm 1 \mathrm{~cm}$ at $500 \mathrm{~cm}$ gives $<0.5 \%$ output variation). If the standard horizontal and vertical transversal laser of the vault are used for positioning of TBI patients, left and right lasers should meet (laterally) at TBI distance within $3 \mathrm{~mm}$.

TSI

To enable irradiation of the entire patient contour, the Stanford technique applies six patient positions (see Figure 4.7). Hence, the patient is rotated each fraction six times to expose different parts of his contour. To improve patient comfort and to gain time, although at the cost of some dose homogeneity, three positions can be irradiated alternately each fraction if the TSI is highly fractionated (e.g. $>6$ fractions). Hence, position 1-3-5 during odd fractions and position 2-4-6 during even fractions. To allow quick and robust patient positioning the angles can be indicated on the floor, e.g. by carving them in the floor. Figure 4.2.b shows the resulting floor pattern. Field line 2 and 3 make a $60^{\circ}$ angle to the guntarget direction,

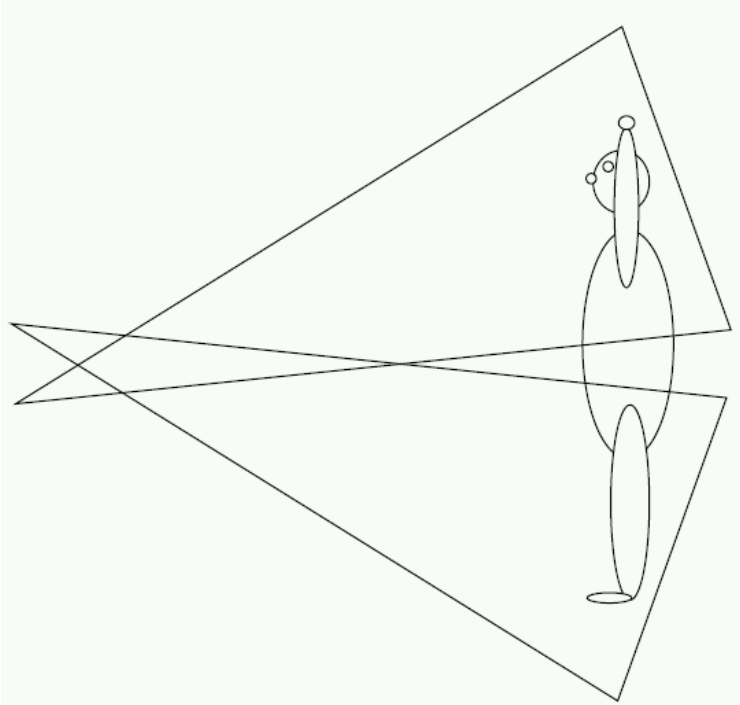

2 gantry angles

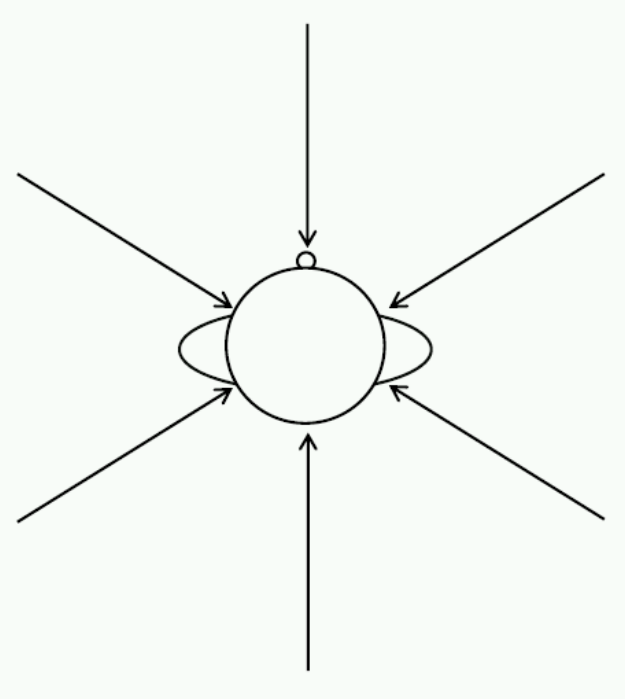

6 patient positions

Figure 4.7 The Stanford technique using 2 gantry angles and 6 patient positions 


\subsubsection{Shielding transmission (TBI only)}

Individual shielding:

In case organs are shielded (e.g. lung, kidney, eye) using (individual) shielding ('blocks') the transmission of this shielding should be determined at TBI distance, ideally at the same off-axis position as they are used clinically to account for off-axis beam hardening/softening. The measurement can be done in a plastic phantom with a cylindrical or parallel-plate ionisation chamber using shielding of different thickness to establish the transmission curve (measure with and without the shielding in position). The transmission curve should be determined for the complete range of expected transmission (so that no extrapolation is necessary). To establish the transmission curve for lung and kidney shielding a shielded area of $10 \times 10 \mathrm{~cm}^{2}$ at the clinically used SSD can be used; for eye lens shielding a cylindrically shielding with diameter of e.g. $3 \mathrm{~cm}$ (projected on the eye) is sufficient. The TPS may provide a means to calculate shielding/compensator transmission. This calculation has to be commissioned.

The measurement depth should be representative for the organs to be shielded. In case a beam spoiler/build-up screen is used clinically, it should be included in the measurement of the transmission curve or modelled in the TPS. A spoiler should be placed within a limited distance (preferably $<20 \mathrm{~cm}$ ) to the patient because the secondary electrons must reach the skin.

\section{MLC-shielding:}

In case a TPS is used, and an MLC is used for shielding, measurements should be done behind leaves and at the leaf edges. Compare measurements and TPS calculations at different positions around both leading and side edges of the MLC leaves, e.g. at $0, \pm 1, \pm 2, \pm 5 \mathrm{~cm}, \pm 10 \mathrm{~cm}$ from the leaf edge at a clinically relevant depth (e.g. $10 \mathrm{~cm}$ ) at the clinically used TBI SSD. Figure 4.8 Absolute dose measurements at TBI distance around the edge of the MLC leaf bank. Dose was measured at $10 \mathrm{~cm}$ depth (Source-detector distance $=350 \mathrm{~cm}$ ) using a standard Farmer chamber in a small dosimetry phantom. One full leaf bank (Elekta Agility) was positioned at a certain distance from the field centre with the leaf bank either blocking the isocentre (positive distance value) or keeping it open (negative value). The graph shows a dose profile corresponding to $500 \mathrm{MU}$, at TBI distance of $350 \mathrm{~cm}$. Measurements are compared to a dose calculation using the standard TPS beam model (Pinnacle, Philips) and a model that was adapted to better match measured dose at TBI distance (courtesy of Radboud University Medical Center, Nijmegen). shows an example of a similar measurement, compared to TPS calculation using the default TPS beam model and a model that was adapted for use in TBI treatments. 
In NCS 22 [107] recommendations are reported for the MLC leaf tip modelling, for the leaf and jaw shielding and the dose calculations outside the field edges. Relative dose differences outside the fields are linked to the modulation factor used in the treatment setup. The difference should be less than \pm $3 \%[103]$.

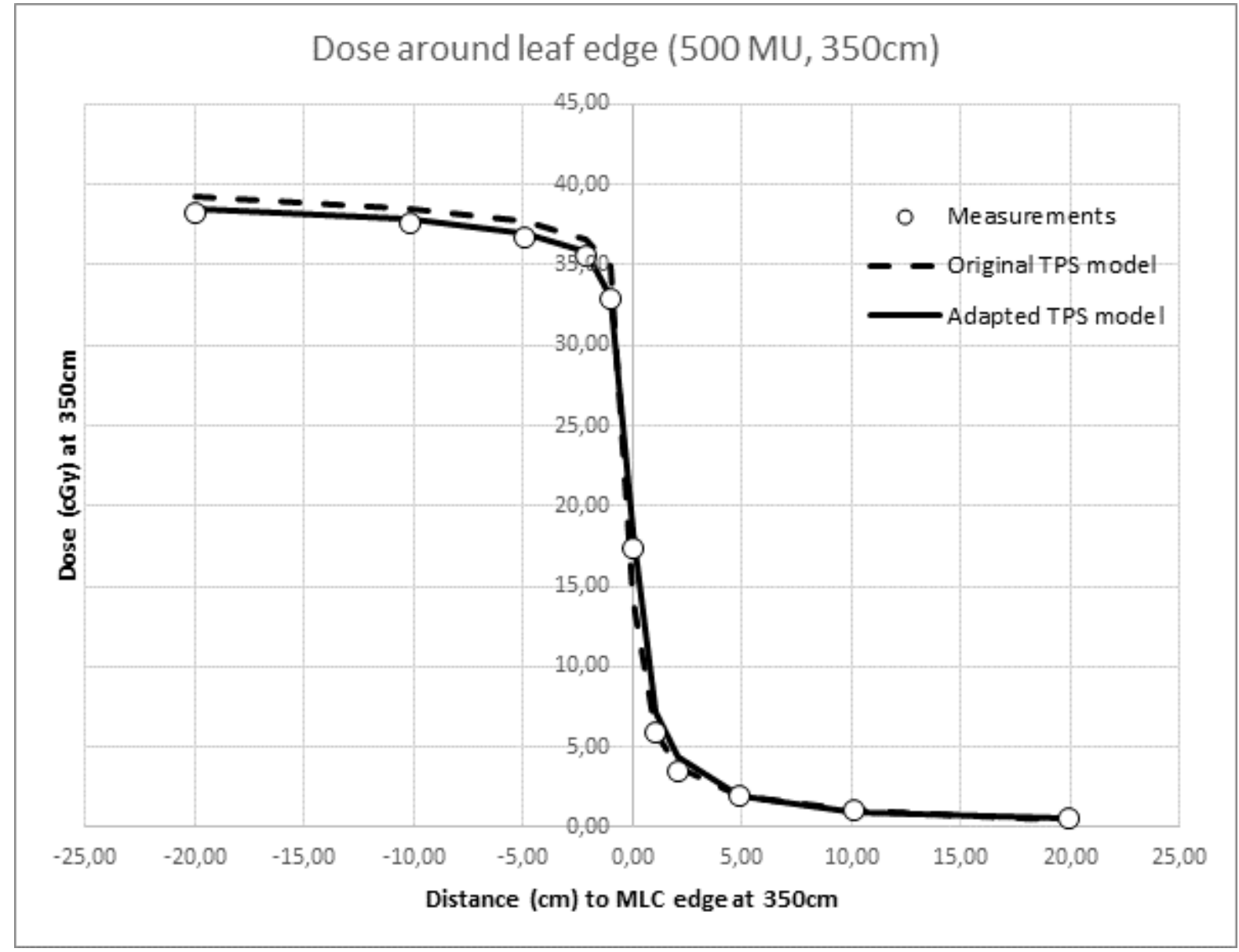

Figure 4.8 Absolute dose measurements at TBI distance around the edge of the MLC leaf bank. Dose was measured at $10 \mathrm{~cm}$ depth (Source-detector distance $=350 \mathrm{~cm}$ ) using a standard Farmer chamber in a small dosimetry phantom. One full leaf bank (Elekta Agility) was positioned at a certain distance from the field centre with the leaf bank either blocking the isocentre (positive distance value) or keeping it open (negative value). The graph shows a dose profile corresponding to $500 \mathrm{MU}$, at TBI distance of $350 \mathrm{~cm}$. Measurements are compared to a dose calculation using the standard TPS beam model (Pinnacle, Philips) and a model that was adapted to better match measured dose at TBI distance (courtesy of Radboud University Medical Center, Nijmegen).

\subsubsection{Extra commissioning steps for TPS workflow (TBI only)}

Using the 'TPS workflow for TBI' as outlined in section 4.2.3 the following parts should be commissioned for clinical usage of a TPS for TBI preparation. 
1. Verify if the maximum field-of-view (scan length, in-plane) of the CT-scanner can fit a patient in treatment position. Often, a custom-made CT-extension table top should be mounted to be able to scan patient from head to toe.

2. Verify if the TPS is able to process whole-body CT datasets. Generally, slice thickness can be increased to, e.g. $10 \mathrm{~mm}$ to decrease data set size.

3. Verify the correct patient orientation in the TPS in case a special patient orientation is used during CT scanning (e.g. in case a patient is positioned right or left decubitus, check if the TPS is able to process such a dataset)

4. If the shaping of the shielding is done from automatically generated lung contours or using scripting: verify the correct shielding shape. Also verify the correct physical shielding dimensions, because treatment planning systems position shielding on 'shadow trays' and the block positioning for TBI can be different from that, e.g. with another SSD. Usually this required some special settings in the block milling device and the TPS.

5. In case a block milling device is used: verify the correct transfer of DICOM RTPlan to block milling device and correct selection of milling device parameters (e.g. magnification factor for SSD)

6. Commission the TPS beam model for TBI calculation:

$0 \mathrm{MU}$ calculation is valid for the extended treatment distance $($ e.g. $S A D=500 \mathrm{~cm}$ ) by verifying the TBI reference output verification against a model of the output phantom in the TPS (deviation calculation vs measurement more than $\pm 3 \%$ [103]. When a beam spoiler screen is used, it should be clear if the screen is included in the TBI reference output or not.

- Verify if the collimator of the linear accelerator (blocks and MLC leaf positions) is modelled correctly for the edges of a 40x40 field in the TPS by measuring penumbra with a spatial resolution of, e.g. $2 \mathrm{~mm}$.

- A special TBI beam model has to be commissioned in case

- another flattening filter is used or if parameters of the clinical beam model has been adapted or tweaked. A flattening filter can be added in the TPS as a block/compensator on a tray.

- the TBI reference output in the TPS has to be adapted when the MU calculation for $\mathrm{TBI}$ reference output is not accurate enough (deviation calculation vs measurement more than $\pm 3 \%$ [103]. 
- The measured beam profile at long SSD deviates from the calculated profile by more than $3 \%$.

- For the validation of the leaf modelling some tests are reported in NCS 22 [107] that can be repeated using the typical TBI SSD distances if modulation of the incident fluency is used.

- In case MLC fields are used, check if the beam model allows table rotations of 180 degrees, in case a table rotation is used to switch between opposite beam directions (e.g. between $A$ and $P$ beam(s)). If not:

- Change the beam model such that it is allowed,

- (in case the treatment vault is sufficiently large) switch to a workflow that involves moving the patient in the bunker rather than rotating the table, or

- Change the beams before import in the R\&V system using TPS-scripting. Example: perform treatment planning with beams from gantry angle 90 ( $\mathrm{A}$ beams) and 270 (P-beams) degrees. Before import in the R\&V system, change all beams to 90 degrees and change collimator angles such that treatment is performed correctly. Proper end-to-end testing of this procedure is mandatory. This method should only be used in combination with a validated script.

7. Is the independent MU calculation accurate enough ( $\pm 5 \%$ ) for the TBI situation? This is to detect gross errors.

8. Correct thickness calculation of the shielding when the required transmission is known.

9. The correct transfer of DICOM RTPlan to R\&V is already commissioned for the standard clinical workflow, but TBI/TSI may involve extreme table positions so some attention has to be paid to this aspect.

10. In case a dedicated TBI portal imager software is used: correct transfer of DICOM Image (DRR with shielding) as reference image.

\subsubsection{Extra measurements for sweeping techniques (TBI only)}

Before a sweeping technique can be used in forward planning manner (without inverse planning), calibration of sweeping technique has to be done using measurements. These measurements consist of: 
a. Beam profile measurements with an ionization chamber positioned mid-plane in a phantom with thickness representative of a clinical thickness (e.g. 18 or $20 \mathrm{~cm}$ thickness) of an unmodulated sweep. This is used to determine the weight distribution of each control point to have a homogeneous dose inside the patient [102].

b. Verification measurements for different phantom thicknesses (range $16-30 \mathrm{~cm}$ ) at different depths to establish the robustness of the correct scaling of the arc segments determined by the measurements done in a).

c. Shielding transmission in a sweeping beam and optimal positioning of the shielding.

d. In-phantom verification measurement to establish the correct scaling of the different segments of the arc in combination with (lung) shielding. This can be done using films and/or TLDs in an anthropomorphic phantom (e.g. Alderson Radiation Therapy phantom).

\subsubsection{Other commissioning items}

a. Dedicated TBI-table: When a dedicated (e.g. an in-house developed) TBI-table is used it should be approved according to the local protocol. Furthermore, it is advised to regularly inspect the condition of this dedicated table, as the use may be infrequent.

b. Reproducibility of gantry position: When the MLC is used, e.g. in a field-in-field technique, the reproducibility of the gantry position should be checked at 90 / 270 degrees. Note that a change of 0,1 degrees in gantry angle moves the centre of the light field with $5 \mathrm{~mm}$ at an SSD of $400 \mathrm{~cm}$, and also the position of MLC segments with respect to the patient changes. Reproducibility of gantry position is generally not a parameter that can be improved, so if variation is too large, e.g. $10 \mathrm{~mm}$ at TBI SSD, the setup protocol should be adapted accordingly. This can be done, for example, by checking the position of MLC fields using the light field prior to irradiation or by ensuring that the dose distributions are robust to positioning errors of up to $10 \mathrm{~mm}$.

c. Influence of distance between beam spoiler and the patient: In the case of TBI the distance between the beam spoiler and the patient is not critical to realise a skin dose $>90 \%$ of Dmax. However, when the distance is large $($ e.g. $>20 \mathrm{~cm}$ ) it should be verified that the skin dose is still appropriate. (see also 4.3.7) 
d. Limitations of the TPS: The treatment planning system should be configured such that the limitations of the linac and the R\&V system are aligned with the limitations of the planning system. For eample, sometimes a $180^{\circ}$ couch rotation is not possible in the TPS and it has to be simulated in the TPS by applying a gantry rotation of $180^{\circ}$ instead of a $180^{\circ}$ couch rotation. In addition, larger SSDs may have to be configured for use with TBI.

e. Backup unit: For TBI, there should always be a (at least one) backup unit available to continue treatment in case of a machine breakdown. This unit has to be commissioned as well. Preferably, the characteristics of the backup machine (e.g. energy, MLC, output within $\pm 2 \%$ ) are equal to the TBI machine to facilitate easy switching between linacs. Apart from the machine characteristics, the patient orientation in the treatment vault is preferably identical. If mirrored vaults have to be used it is important to have a protocol or a backup plan converting the gantry and collimation angles. Besides, it must be possible to mount shielding and spoiler screens in the same way. When linacs are not completely equal (e.g. because of different MLC systems, other reference output, different vault orientation) a detailed local protocol should be available, describing how to continue the treatment correctly and safely. For TSI, timing is less critical, so a backup unit is not essential. Furthermore, precautions should be taken as well for peripheral items, e.g. the portal imager and the treatment table.

\subsubsection{Cardiac Implantable Electronic Device and other electronic devices (TBI/TSI)}

In case the patient presents with a Cardiac Implantable Electronic Device (CIED), precautions need to be taken according to the guidelines laid out by the AAPM Taskgroup 203 report [108]. In case of TBI sparing of the device is very difficult by nature of the technique. In case of TSI the radiation oncologist may decide to spare the CIED by shielding it with e.g. Pb. Other electronic devices such as neurostimulators have to be treated with due caution, along the same line as used for CIEDs. 


\section{Pre-treatment workflow, treatment delivery and in-vivo verification}

\subsection{Pre-treatment workflow of TBI and TSI}

\subsubsection{Prelude}

Each paragraph describes a different workflow. This implies that some paragraphs are similar when needed in the specific workflow. For TSI treatments, the manual calculation workflow is the only available and validated method.

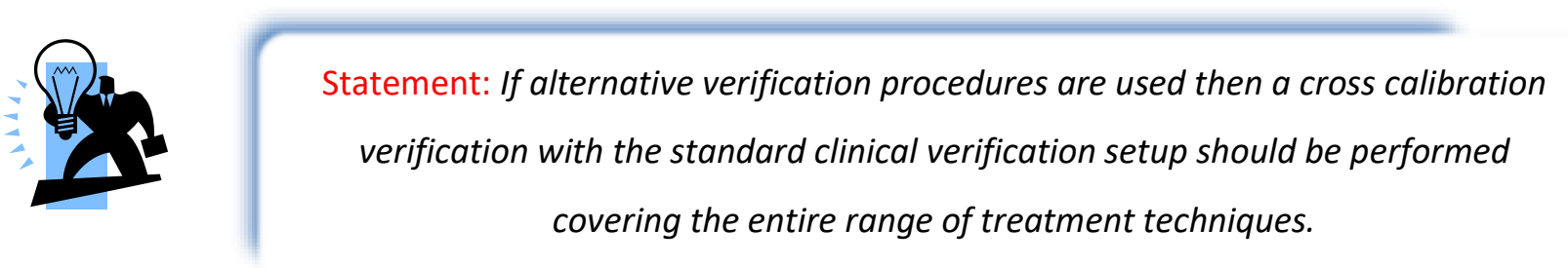

\subsubsection{The manual calculation workflow}

Transfer of MUs to the Record and Verify (R\&V) system for TBI/TSI: manual input

When the MUs are calculated on the dose reference points [38] using a dedicated (mostly home-made) calculation program, the MUs have to be entered manually into the R\&V system of the treatment machine. It is recommended to design homemade calculation and transfer programs such that all relevant beam parameters (MUs, energy, field size) can be sent to the Record \& Verify, thereby avoiding manual entering of beam parameters into the $R \& V$ system.

For some vendor/machine types, the number of MUs per beam is limited (for instance to 990 or 1000). Beams exceeding this number of MUs then have to be split into multiple static beams with $1000 \mathrm{MUs}$ or using segments of an IMRT beam. For safety reasons it is not recommended to treat multiple times with one beam from the R\&V system since this may lead to mistreatment: too few or too many beams may be given by mistake. Furthermore, with multiple beams the treatment can be scheduled and registered properly.

In order to avoid errors in preparing a manual prescription, preferably a template should be available in the database of the R\&V system or should be imported into the R\&V system. We advise that for each dose prescription a separate template should be available. The correct template can be loaded 
and linked to the patient to ensure a correct treatment prescription so that only a manual input of required MUs is needed.

Treatment verification must be performed using the QA mode of the treatment verification software, allowing detection of treatment delivery parameters, but without dose registration of the verification fields. An independent MU calculation or measurement and, if used, attenuator thickness calculation or measurement prior to start of the treatment is necessary [38].

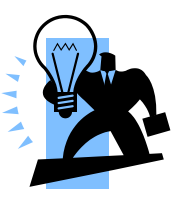

Warning: Treatment should NEVER be performed in a non-clinical mode of the treatment machine (e.g. 'service mode'). Although it seems to be practical, e.g. because the limitation on the mentioned maximum number of MUs can be avoided in that mode or the applicator can be overwritten for TSI treatments..., the interlock system in that mode is insufficient for safe patient treatment (and it is also not legally allowed to do so). Only clinical mode treatments certify a dose delivery in agreement with dose prescription, and a correct administration of the applied dose. Pretreatment checks must be performed in QA mode.

Transfer of shielding parameters to the block-milling machine for TBI treatments For standing and sitting patient setups no CT-based treatment planning workflow is possible. When dose reduction is needed to reduce the dose to critical organs, a treatment simulation process is necessary in order to determine the dimensions and positioning of the shielding blocks and spoiler/build-up screens. Image registration using aSi detector plates or other digital detection techniques and radiosensitive films can be used to visualise the shielding with respect to the patient anatomy in order to determine shape and size of the blocks. Because usually the blocks are nearby the patient the large SSD distance divergence is not important in milling/pouring the blocks. The SSD distance is almost equal for each patient, so that the magnification factor is well-known and (more or less) patient independent. The block coordinates are transferred to the block-milling machine using a stereoscopic cutter system or by using a digital transfer. Field centre coordinates are often included to align the blocks within tolerances with the block holder. Pre-treatment verification of the size and position can be performed using a print of the detector plate/radiosensitive film and the light field of the linac on an empty block holder. The thickness of the shielding blocks is determined using transmission data of the block material. Mostly custom-made calculation programs are used to predict the block thickness in order to achieve the desired dose absorption. The thickness of the shielding blocks is realised by pouring the correct amount of liquid shielding material into the Styrofoam mould, 
which is cut by the block-milling machine. The resulting shielding blocks should be checked afterwards independently prior to treatment (either by measurement of the actual block transmission or by measuring the actual thickness).

As an alternative for a block-milling machine, shielding can also be made by cutting lead sheets by hand using special scissors. Lead sheets can be stacked to obtain the required thickness.

Scripting/tabulating can be used as alternative verification procedure after cross calibration with standard verification.

\subsubsection{Transfer of shielding parameters to the block-milling machine for TBI treatments}

Transfer a complete DICOM-RTPlan from the TPS to the R\&V-system including MUs.

If the TBI treatment plan is created using a TPS, the total number of MUs to be given can be transferred via DICOM-RTPlan to the R\&V system. The DICOM-RTPlan is imported using the standard clinical procedure. See also section 4.3.10,

Transfer of Block shielding from the TPS to the block-milling machine If individualized shielding blocks are used to reduce OAR dose e.g. the lung dose, these blocks are preferably fabricated using a block-milling machine. For the large SSD (e.g. $500 \mathrm{~cm}$ ) at which TBI is executed, it is usually not necessary to have divergent blocks. The shape of the shielding blocks is usually determined with a TPS based on the patient CT. The block coordinates are preferentially transferred from the TPS to the block-milling machine using DICOM-RTPlan. The thickness of the shielding blocks is realised by pouring more or less liquid shielding material into the Styrofoam mould, which is cut by the block milling machine. The resulting shielding blocks should be checked afterwards independently (either by measurement of the actual block transmission or by measuring the actual thickness and using the established block transmission curve).

As an alternative for a block-milling machine, shielding can also be made by cutting lead sheets by hand using special scissors. Lead sheets can be stacked to obtain the required thickness.

\subsubsection{Shielding block transmission and compensator transmission under TBI-conditions}

Transmission coefficients of the (lung) shielding should be verified at least for the first 10 patients [107] using an identical setup as during the TBI treatment, including the spoilers/build-up screens (if applicable). Therefore, a measurement at a lung-equivalent depth mid-plane with and without the 
block using identical detectors as for the absolute dose calibration (see section 4.3.1). If alternative verification procedures are performed, cross calibration verification with the clinical setup must be performed covering the entire range of patients. If modelled in the TPS, the block transmission should be commissioned separately.

Compensators can be used to modify the homogeneous entrance beam to compensate for missing tissue allowing dose homogeneity within $\pm 10 \%[38,109]$. Also, bolus material can be used as compensator material to increase dose homogeneity for instance around the neck and ankles [54]. High-energy beams in AP-PA orientation are less affected because this setup is less sensitive to dose inhomogeneity (see section 4.3.3).

\subsection{Treatment delivery}

\subsubsection{Recommendations for single and multiple fraction treatment delivery for TBI/TSI}

Set-up errors during treatment should be minimised. Therefore, additional markers and lasers can be used to (re)position the patient within acceptable tolerances $( \pm 1 \mathrm{~cm})$. Prior to treatment delivery additional checks for the exact positioning should be performed: the positioning of the table/stand, the positioning of the patient, shielding blocks and spoilers. The alignment of the patient/shielding blocks with respect to the treatment beam can be done using traditional radiographic films or digital imaging devices, while optical guidance of this alignment is inaccurate and outside clinical tolerances.

Together with patient position assisting aids in the treatment setup, rotation errors larger than $1^{\circ}$ can be avoided resulting in set-up accuracy in position to be within $1 \mathrm{~cm}$ [38]. When using an MLC-based treatment plan (only for TBI), a dedicated beam segment shaped according to the patient contour can be used to position the patient using the light field projected by this MLC field. MLC fields encompassing one or both lungs can also help to verify patient's alignment for TPS based treatment delivery by using film/digital imaging [110].

Regular inspection of the structural integrity of the spoiler screens and the patient support is necessary in order to identify any cracks, pits or other imperfections that may affect its use in mechanical stability or in behaviour of the induction of build-up or scatter. 
The patient is monitored during treatment through a video system (or a similar alternative) and audio contact should be used to ensure the patient remains in the correct position during the treatment. This is in particular important for patients who are usually more mobile during treatment, especially if they are not under anaesthesia.

\subsubsection{Recommendation for treatment verification in multiple fraction treatment delivery}

An online treatment verification is the preferred method for treatment verification, also for multiple fraction treatment delivery, for instance by using electronic portal imaging.

When offline review is performed during previous treatment fractions, the observed differences should be evaluated. Tolerances for non-intervention should be discussed in advance and when deviations are exceeding those tolerances, a modification strategy should be agreed on prior to the start of the TBI treatment process. A correction of half or $2 / 3$ of the observed deviation is recommended in order to avoid overcompensation and to converge to the optimal treatment set-up.

Some TSI fractionation schemes allow to deliver half of the fields on odd treatment sessions and half on even treatment sessions (see also section 4.3 .6 and $[67,111]$. This implies validation of both sessions separately.

\subsubsection{In-vivo dosimetry}

The rationale for in-vivo dosimetry is two-fold:

a. documentation and verification of the actual treatment;

b. evaluation of (adapted) treatment technique for the first 10 patients or more.

In the survey (chapter 3 ) for TBI about $50 \%$ of the institutions in the Benelux countries performed invivo dosimetry. TLDs, diodes, MOSFETs are used to verify the delivered dose at the umbilicus and/or to measure the doses at body extremities (feet, hands, head) or at the thorax (lungs). Entrance and exit doses are used to determine the central line dose for those positions (See also section 4.3.4). When high doses are delivered over the entire treatment, the dose could be monitored in real-time (using diodes) or integrated for an entire fraction (using TLDs, MOSFETs, Semiconductor, lon chamber, films) $[59,88]$. The first methods facilitate online adjustment of the patient setup, whereas the second allows for post-irradiation processing analysis and treatment adjustment. Still, using e.g. MOSFETs (a non- 
real-time detector) it is also possible to monitor a part of the complete treatment and analyse its reading. This may lead to an on-line intervention if an error is detected. When a field-in-field or IMRT technique is used this monitoring can be done on a part of the first (e.g. largest) segment. The tolerances for deviations between predicted values and measured values is set between $5-10 \%$ as a warning level [94]. For intervention in TBI, a pragmatic level of $15 \%$ of the prescribed dose (including boost fields) could be employed [54].

For TSI, in-vivo treatment verification is reported using similar detectors/films as for TBI treatments [63]. Dose differences between expected and measured doses commonly vary significantly exceeding the $10 \%$ threshold confirming the less predictive value of dose in TSI versus TBI treatments. A deviation of $20 \%$ is still acceptable for this treatment modality $[44,111]$.

The total number of treatments differ from centre to centre and low numbers on yearly basis are reported (especially for TSI). Therefore, pre-treatment functionality testing and calibration verification of the in-vivo system is recommended, noting that the accepted deviations differ from those used for standard in-vivo conditions. 


\section{Risk Analysis in TBI and TSI}

\subsection{Introduction}

A reliable QC procedure is a very helpful method to guarantee that medical devices operate in a predictable and safe fashion. However, proper commissioning of the equipment used for TBI and TSI treatment (including preparation and calculation) alone does not guarantee that in the clinical workflow all individual steps are completely safe. It is well accepted that a (prospective) risk assessment is a valuable tool in a patient safety management system.

The prospective risk analysis as described in this chapter comes on top of the standard risk analysis as described in detail in chapter 5 of NCS-report 22 [107].

To ensure a safe introduction and application of TBI and TSI procedures, the entire clinical workflow has to be analysed for possible hazardous process-steps.

According to NCS-22 the goals of a risk analysis are:

- $\quad$ Designing a workflow for preventing incidents and accidents

- $\quad$ Obtaining insight in the complexity of the process

- $\quad$ To have a basis to design a QA/QC program and to implement safety checks specifically targeted at the vulnerable steps of the process tree

Prospective risk analysis can be done using HFMEA (Healthcare Failure Mode and Effect Analysis). See De Rosier for details [86]. In 2006, a Dutch translation of HFMEA was adapted to the Dutch healthcare situation and made it more practical. This adaption was called SAFER (Scenario Analyse van Faalwijzen, Effecten en Risico's). In Belgium HFMEA or AMDEC (Analyse des modes de défaillance, de leurs effets et de leur criticité), the French translation of Failure Modes, Effects and Criticality Analysis (FMECA), will be implemented in the near future. The risk analysis in NCS report 22 is performed following SAFER because of its applicability to improve patient safety and because it is commonly accepted and applied in Dutch hospitals as part of a safety management system. The best information of SAFER can be found in NCS report 22, because online information is difficult to find, although a Dutch reference can be found by VMS-Zorg [87]. 
The risk analysis presented in NCS-22 is the basic analysis, which should always be performed. The extension presented in this chapter comes on top of this basic analysis and will focus on the process steps to identify risks for TBI and TSI treatment specifically.

\section{Privacy Impact Assessment}

Due to the European General Data Protection Regulation (GDPR, in Dutch: AVG) special attention should be given to a Privacy Impact Assessment (PIA) and BIV-classification. In Dutch this is referred to as 'Beschikbaarheid, integriteit en vertrouwelijkheid' (BIV), see Wikipedia [112]; in English it is named CIA triad, confidentiality, integrity and availability (CIA) [113].

Specifically for the manual workflow one should analyse if personal data is used which is traceable to an individual.

\subsection{Prospective Risk analysis in TBI and TSI}

\subsubsection{General aspects and the modified risk inventory matrix}

The general goal of a prospective risk analysis is to identify risk factors for a specific treatment technique or process, in this chapter: TBI and TSI treatments. Identified risks can be divided into two groups:

- $\quad$ Failure modes in the process of developing and introducing a TBI/TSI technique

- $\quad$ Failure modes in the process branch of the TBI/TSI clinical workflow

In Figure 6.1 for the failure mode a frequency and severity score is shown according to the risk inventory matrix. In this figure the frequency category 1 corresponds to less than once a year, whereas 4 corresponds to a weekly occurrence. For severity, category 1 corresponds to minor consequences and 4 to catastrophic ones. For application in radiotherapy, catastrophic means adverse clinical effects for one or more patients, e.g. with deviations from the intended dose of $20 \%$ (see NCS-22, page 69 [107]).

Each group is presented in a two-dimensional matrix, frequency vs. severity. Each cell in the matrix refers to an identified risk. Frequent risks with a high severity are located in the upper left part of the matrix, and infrequent risk with little/minor severity are shown in the lower right part. The red-coded cells (High and Very high) are risks that need to be addressed. 


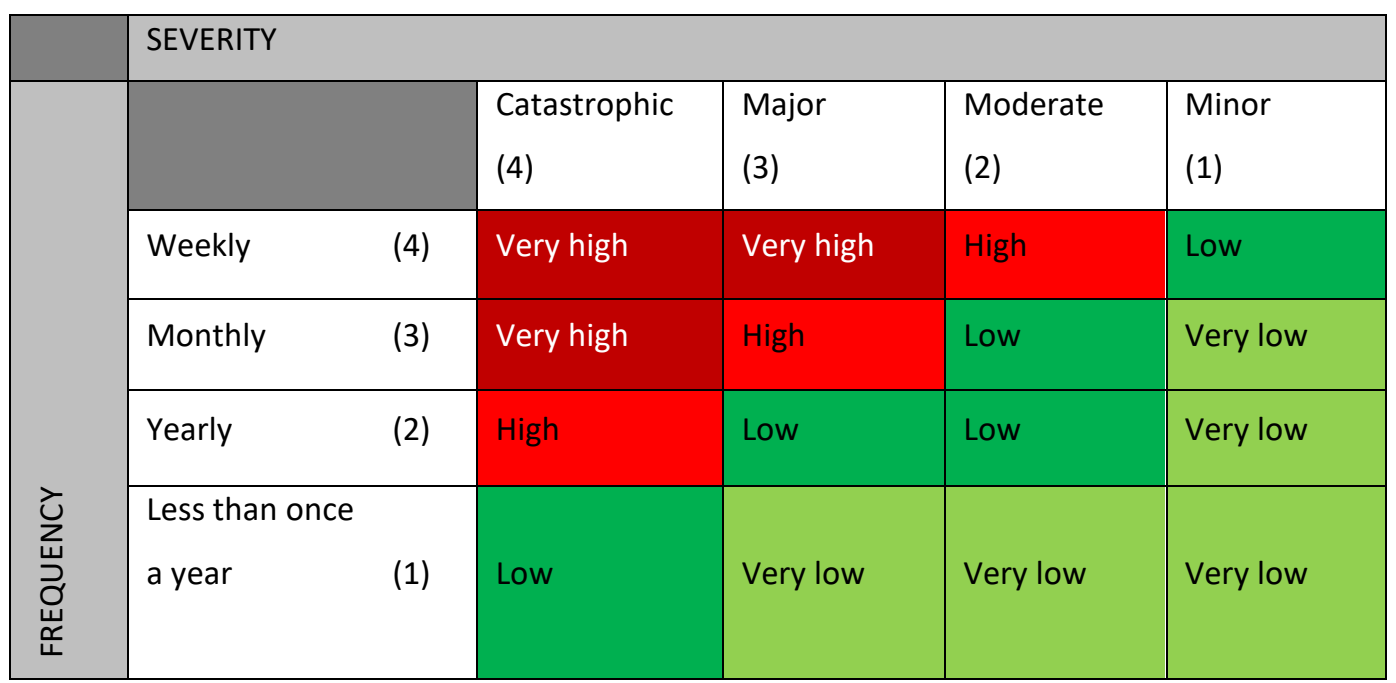

Figure 6.1 Risk inventory matrix. The frequency (indicated between brackets) and severity category of a failure mode determine the risk score[107]

However, for the small number of TBI/TSI patients/treatments in an individual centre the matrix of Figure 6.1 is unpractical with respect to the frequency of a failure mode. In such cases, it is better to specify the probability of a failure as the number of events per patient / treatments. In Figure 6.2 below, this probability is shown as the event per patient/treatments. For example, 1/1 means 1 event occurs for each patient/treatment, 1/2: 1 event per 2 patients/treatments, 1/20: 1 event per 20 patients/treatments.

Also the severity can be specified in more detail:

- Minor: the event remains unnoticed for the patient with no or minor effect on the resulting treatment

- Moderate: small discomfort for the patient and / or small effect on the resulting treatment

- Major: effecting the treatment outcome with a possible complaint of the patient; (extreme) dissatisfaction of the patient. The effect may also result in dysfunction of personnel.

- $\quad$ Catastrophic: legal violations; severely compromise treatment outcome; possible injury to (or death of) patients or personnel.

In the following two paragraphs the failure modes for the TBI and TSI clinical workflow will be addressed. 


\begin{tabular}{|c|c|c|c|c|c|}
\hline & \multicolumn{5}{|l|}{ SEVERITY } \\
\hline \multirow{5}{*}{ 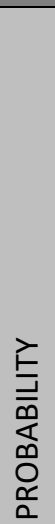 } & & $\begin{array}{l}\text { Catastrophic } \\
\text { (4) }\end{array}$ & $\begin{array}{l}\text { Major } \\
\text { (3) }\end{array}$ & $\begin{array}{l}\text { Moderate } \\
\text { (2) }\end{array}$ & $\begin{array}{l}\text { Minor } \\
\text { (1) }\end{array}$ \\
\hline & $1 / 1$ or $1 / 2$ & Very high & Very high & High & Low \\
\hline & $1 / 5 \ldots 1 / 10$ & Very high & High & Low & Very low \\
\hline & $1 / 20 \ldots 1 / 50$ & High & Low & Low & Very low \\
\hline & $1 / 100 \ldots$ seldom (1) & Low & Very low & Very low & Very low \\
\hline
\end{tabular}

Figure 6.2 Modified risk inventory matrix for a limited number of patients/treatments. The probability is given as the event per patient. The probability and severity category of a failure mode determine the risk score. The numbers between brackets () are used in Table 6.1, Table 6.2 and Table 6.3.

\subsubsection{Examples of failure modes in the TBI/TSI development and introduction}

The risk analysis in the process of developing and introducing a TBI/TSI technique is not addressed here, because it is not different from developing any other treatment technique. See NCS-22 for more information.

\subsubsection{Examples of failure modes in the TBI clinical workflow}

Usually prior to clinical practice of TBI or TSI, a multi-disciplinary investigation should be performed. In the list below, specific items are given in the workflow for TBI and possible failure modes (in italic). This table is only a guideline in helping to identify possible failure modes and should always be adapted to the local situation.

In Table 6.1 and Table 6.2, these failure modes are analysed and classified with possible solutions. The failure modes in grey are not included in these tables. The numbers between brackets refer to the corresponding rows.

- Patient specific shielding (lung, eye, kidney)

- Shielding from another patient is used (1a)

- Standard shielding (e.g. eye)

- Standard shielding is accidentally forgotten or translates / gets loose during treatment(1b) 
- Transmission blocks that are manually positioned

- The blocks can drop down and possibly injure e.g. an RTT or a patient $(2 a, 2 b)$

Blocks can fall from their position plates $(2 a, 2 b)$

- Blocks are swapped or missing (2c)

- Use of a block milling device

- The milling device is not properly commissioned for TBI

- The wrong block prescription is used (in case the block milling device is used only for $T B I)$

- The wrong block thickness is poured (1d)

- Blocks with air bubbles go undetected (1c)

- Wrong thickness calculated/wrong HVL in TPS (1d)

- Wrong correction of scaling/divergence as a result of the position of the block between patient and isocentre (1e)

- Wrong block-beam combination is used (1f)

- Dedicated movable couch or positioning/fixation stand (often home-made)

- The couch can be positioned on a wrong SSD-position or height (3a)

- Shifting of patients for AP / PA irradiation

- There is a risk of falling (dedicated couch/table/patient) (in case an non-standard setup/device is used for TBI) (3c)

- Blocks are interchanged or missing (for instance PA block with AP blocks) (4a)

- Extra positioning lasers (not for the sweeping technique)

- The extra positioning lasers are not checked frequently (5a)

- Separate beam spoiler

- The beam spoiler is not used (6a)

- The beam spoiler is positioned at a wrong distance (6b)

- Extreme table positions

The extreme table position gives an inhibit for the treatment (due to an interlock or the table in the beam pathway)

- Use of a separate movable portal imager (or self-developing films) in combination with DRRs from the TPS

- The wrong reference image is used to evaluate the shielding position/shape (7a)

- The portal imager is positioned on a wrong position (7b) 
- The electronics of the portal imager is damaged by using it in the complete treatment beam (7c)

- Patient positioning

- Patient was positioned incorrectly (8a)

- Special TBI beam model (in case necessary)

The beam is not configured/commissioned correctly (10a)

- The wrong beam model is selected during treatment planning (10b)

- Long treatment times

- Anatomy changes due to sagging of the patient (8b)

- Patient can get ill during the treatment; the treatment has to be interrupted to take care of the patient; the correct continuation of the treatment should be exercised in combination with $R \& V$ system (13a)

- Dose calculation

- Patient dimensions are measured at wrong position, or are swapped (9a)

- Patient dimensions are entered wrongly in dose calculation program or spreadsheet. (9b)

- Other errors in dose calculation (wrong energy, SSD, etc.) (9c)

- Extreme anatomies: 'pointy belly' with umbilical dose definition can lead to high doses in extremities (9d)

- Data transfer

- Beam parameters are transferred wrongly to R\&V system or linac especially with manual transfer. (11a)

- Multiple beams for large number of MUs

- not all beams are created in the R\&V-system (12a)

- not all beams are registered in the $R \& V$-system (12a)

- not all beams are used in treatment (12a)

- Critical timing radiation schedule and transplantation date.

- linac break down, but radiation treatment should be continued and finished (13b)

- institutional power failure (e.g. emergency power test) or a major TBI machine upgrade), also no backup machine is available (13c)

- In-vivo dosimetry

- Errors in measurement (wrong channels, measurement started/stopped too late/early, wrong calibration factors) $(14 a, 14 b)$ 
- Errors in positioning of dosimeters (14b)

○ Measurement forgotten (14c)

Specific items in the workflow for TBI with the sweeping technique are:

- Two CT-scans (supine and prone)

- Treatment is based on thickness in one CT scan instead on both CT-scans (15a)

- Generic arc is used

- The arc is not adapted correctly for the individual patient thickness (16a)

Table 6.1 Risk analysis for TBI treatment at extended SSD. With severities (S) ranging from minor (1) to catastrophic (4) (see definitions in Figure 6.2). Probabilities (P) ranging from 1 out of 100 patients (seldom) (1) to 1 out of 2 patients (4). Resulting risk scores (RS): Very Low (VL), Low (L), High (H) and Very High (VH).

\begin{tabular}{|c|c|c|c|c|c|}
\hline Process description & $\begin{array}{l}\text { Possible failure mode / } \\
\text { consequence }\end{array}$ & $\mathbf{S}$ & $\mathbf{P}$ & RS & Possible solution \\
\hline \multirow{6}{*}{$\begin{array}{l}\text { 1. Patient specific } \\
\text { shielding } \\
\text { (lung/eye/kidney) }\end{array}$} & $\begin{array}{l}\text { a. Shielding from another } \\
\text { patient is used }\end{array}$ & 3 & 1 & VL & Labelling \\
\hline & $\begin{array}{l}\text { b. Standard shielding } \\
\text { accidentally forgotten or } \\
\text { shifts / get loose during } \\
\text { treatment }\end{array}$ & 3 & 2 & L & $\begin{array}{l}\text { Remark in patient setup of } R \& V \text {; observe } \\
\text { visually during treatment. }\end{array}$ \\
\hline & $\begin{array}{l}\text { c. Failure shielding due to } \\
\text { air bubbles in shielding }\end{array}$ & 3 & 1 & $\mathrm{VL}$ & $\begin{array}{l}\text { Pre-treatment check for first } 10 \text { patients } \\
\text { (or weight the shielding and compare } \\
\text { weight based on physical dimensions). }\end{array}$ \\
\hline & $\begin{array}{l}\text { d. Failure shielding due to } \\
\text { wrong block thickness (e.g. } \\
\text { originating from wrong } \\
\text { HVL TPS or wrong pouring) }\end{array}$ & 3 & 1 & $\mathrm{VL}$ & $\begin{array}{l}\text { Pre-treatment check for first } 10 \text { patients; } \\
\text { derive probable ranges using a } \\
\text { verification list.. Check by dedicated TBI } \\
\text { team. }\end{array}$ \\
\hline & $\begin{array}{l}\text { e. Failure shielding due to } \\
\text { wrong scaling }\end{array}$ & 3 & 1 & VL & $\begin{array}{l}\text { Use fixed distances and dedicated TBI } \\
\text { team; visual inspection of position of the } \\
\text { blocks on the tray holder and/or } \\
\text { inspection of the light field projection; } \\
\text { portal imaging/ film in combination with } \\
\text { DRR. }\end{array}$ \\
\hline & $\begin{array}{l}\text { f. Wrong shielding-beam } \\
\text { combination }\end{array}$ & 3 & 1 & $\mathrm{VL}$ & Use correct labelling. \\
\hline \multirow[t]{3}{*}{$\begin{array}{l}\text { 2. Positioning blocks by } \\
\text { hand }\end{array}$} & $\begin{array}{l}\text { a. Blocks can fall from } \\
\text { positioning plates and } \\
\text { injure e.g. a RTT }\end{array}$ & 3 & 2 & L & $\begin{array}{l}\text { Set up a dedicated TBI team and practise } \\
\text { in a safe environment. }\end{array}$ \\
\hline & $\begin{array}{l}\text { b. Blocks can fall from } \\
\text { positioning plates and } \\
\text { injure a patient }\end{array}$ & 3 & 1 & $\mathrm{VL}$ & $\begin{array}{l}\text { Position blocks at a distance from the } \\
\text { patient; position blocks at the non- } \\
\text { patient side of the tray holder. }\end{array}$ \\
\hline & $\begin{array}{l}\text { c. Blocks are swapped or } \\
\text { missing }\end{array}$ & 3 & 1 & $\mathrm{VL}$ & $\begin{array}{l}\text { Set up a dedicated TBI team and mark } \\
\text { the blocks clearly; visual check during the } \\
\text { first session using the light field } \\
\text { projection. }\end{array}$ \\
\hline $\begin{array}{l}\text { 3. Dedicated movable } \\
\text { couch or positioning/ }\end{array}$ & $\begin{array}{l}\text { a. Couch can be positioned } \\
\text { at wrong SSD or height }\end{array}$ & 3 & 2 & L & $\begin{array}{l}\text { Use couched linac table and R\&V as } \\
\text { fixation stand for shielding. }\end{array}$ \\
\hline
\end{tabular}




\begin{tabular}{|c|c|c|c|c|c|}
\hline $\begin{array}{l}\text { fixation stand (often } \\
\text { home-made) }\end{array}$ & & & & & $\begin{array}{l}\text { Set up a dedicated TBI team and use a } \\
\text { fixed position procedure. }\end{array}$ \\
\hline & $\begin{array}{l}\text { b. Wrong couch } \\
\text { positioning/fixation stand } \\
\text { is not positioned properly } \\
\text { (in size and/or correction) }\end{array}$ & 3 & 3 & $\mathrm{H}$ & $\begin{array}{l}\text { Use couched linac table and R\&V; use a } \\
\text { (homemade) stand with read-out } \\
\text { position verification tools. }\end{array}$ \\
\hline & $\begin{array}{l}\text { c. Risk of falling (couch } \\
\text { with patient) during } \\
\text { movement of the } \\
\text { dedicated couch }\end{array}$ & 4 & 2 & $\mathrm{H}$ & $\begin{array}{l}\text { Setup a dedicated TBI-team; use a robust } \\
\text { device and do not move with patient } \\
\text { positioned in the device. }\end{array}$ \\
\hline $\begin{array}{l}\text { 4. Blocks are } \\
\text { interchanged (e.g. PA - } \\
\text { AP block interchange) }\end{array}$ & a. Failure shielding & 3 & 2 & $\mathrm{~L}$ & $\begin{array}{l}\text { Portal imaging/film combined with DRR; } \\
\text { visual inspection of the light field of the } \\
\text { linac with blocks on tray holder. }\end{array}$ \\
\hline 5. Patient positioning & $\begin{array}{l}\text { a. Wrong positioning due } \\
\text { to offset TBI laser(s) }\end{array}$ & 2 & 1 & VL & $\begin{array}{l}\text { Regular QA, or } 100 \% \text { pre-treatment QA } \\
\text { on TBI lasers (e.g. using reference } \\
\text { markers on the wall); TBI stand markers } \\
\text { on the floor. }\end{array}$ \\
\hline \multirow[t]{2}{*}{ 6. Beam spoiler } & $\begin{array}{l}\text { a. Dose failure due to TBI } \\
\text { without beam spoiler }\end{array}$ & 3 & 2 & L & $\begin{array}{l}\text { Set up a dedicated TBI team; use in-vivo } \\
\text { dosimetry. }\end{array}$ \\
\hline & $\begin{array}{l}\text { b. Wrong positioning beam } \\
\text { spoiler }\end{array}$ & 3 & 1 & VL & $\begin{array}{l}\text { Set up a dedicated TBI team; use a label } \\
\text { to mark position of beam spoiler. }\end{array}$ \\
\hline \multirow[t]{3}{*}{ 7. Portal imaging } & $\begin{array}{l}\text { a. The wrong reference } \\
\text { image is used to evaluate } \\
\text { the shielding } \\
\text { position/shape }\end{array}$ & 3 & 2 & $\mathrm{~L}$ & $\begin{array}{l}\text { Set up a dedicated TBI team and use a } \\
\text { detailed manual for this procedure; label } \\
\text { the reference image with patient initials } \\
\text { using lead letters. }\end{array}$ \\
\hline & $\begin{array}{l}\text { b. Wrong correction due to } \\
\text { wrong positioning imager }\end{array}$ & 3 & 2 & $\mathrm{~L}$ & $\begin{array}{l}\text { Make a check image after repositioning } \\
\text { blocks; verification using light field on } \\
\text { patient itself after correction. }\end{array}$ \\
\hline & $\begin{array}{l}\text { c. Damage electronics due } \\
\text { to treatment beam }\end{array}$ & 3 & 2 & $L$ & $\begin{array}{l}\text { Interlock between linac and imager may } \\
\text { prevent this; use a verification beam with } \\
\text { smaller field sizes to prevent irradiation } \\
\text { of the electronics }\end{array}$ \\
\hline \multirow[t]{2}{*}{ 8. Patient positioning } & $\begin{array}{l}\text { a. Failure due to wrong } \\
\text { patient positioning }\end{array}$ & 4 & 3 & $\mathrm{VH}$ & $\begin{array}{l}\text { Set up a dedicated TBI team; label the } \\
\text { position of the specific patient on the } \\
\text { positioning device using skin marks and } \\
\text { marks on the positioning device. }\end{array}$ \\
\hline & $\begin{array}{l}\text { b. Failure due to sagging } \\
\text { patient during long } \\
\text { treatment times }\end{array}$ & 3 & 3 & $\mathrm{H}$ & $\begin{array}{l}\text { Assure maximal as possible patient } \\
\text { comfort, and use max dose-rate; indicate } \\
\text { and check initial positioning using video; } \\
\text { observe patient using video system; } \\
\text { visual inspection of the patient position } \\
\text { between beams; integrated imaging. }\end{array}$ \\
\hline \multirow[t]{2}{*}{ 9. Dose Calculation } & $\begin{array}{l}\text { a. Patient dimensions are } \\
\text { measured at wrong } \\
\text { position, or are swapped }\end{array}$ & 4 & 2 & $\mathrm{H}$ & $\begin{array}{l}\text { Apply CT-based planning enabling a } \\
\text { check; derive probable MU range as an } \\
\text { independent check; put patient } \\
\text { dimensions in the field notes in the R\&V } \\
\text { system and, visualize them in the bunker } \\
\text { as an independent check. Put verification } \\
\text { of the credibility of the data in the } \\
\text { protocol. }\end{array}$ \\
\hline & $\begin{array}{l}\text { b. Patient dimensions are } \\
\text { entered wrongly in dose } \\
\text { calculation program or } \\
\text { spreadsheet }\end{array}$ & 4 & 2 & $\mathrm{H}$ & $\begin{array}{l}\text { Apply CT based planning avoiding hand } \\
\text { input and enabling an independent check } \\
\text { (e.g. use scripting possibilities of the } \\
\text { TPS). }\end{array}$ \\
\hline
\end{tabular}




\begin{tabular}{|c|c|c|c|c|c|}
\hline & $\begin{array}{l}\text { c. Other errors in dose } \\
\text { calculation (wrong energy, } \\
\text { SSD, etc.) }\end{array}$ & 4 & 1 & $\mathrm{~L}$ & $\begin{array}{l}\text { Apply a class solution (with a fixed } \\
\text { energy and SSD etc.) and enabling an } \\
\text { independent check (e.g. use scripting } \\
\text { possibilities of the TPS). }\end{array}$ \\
\hline & $\begin{array}{l}\text { d. Extreme anatomies: } \\
\text { 'pointy belly' with } \\
\text { umbilical dose definition } \\
\text { can lead to high doses in } \\
\text { extremities and head }\end{array}$ & 3 & 2 & $\mathrm{~L}$ & $\begin{array}{l}\text { Use CT based planning or determine the } \\
\text { dose based on more than one cross- } \\
\text { section; apply field in field or segmented } \\
\text { fields; correction using a compensator } \\
\text { (e.g. solid water block). }\end{array}$ \\
\hline \multirow{2}{*}{$\begin{array}{l}\text { 10. Special TBI beam } \\
\text { model }\end{array}$} & a. Wrongly commissioned & 4 & 1 & $\mathrm{~L}$ & Pre-treatment check for first 10 patients. \\
\hline & b. Wrong model used & 4 & 1 & L & $\begin{array}{l}\text { Set up a dedicated TBI team; choose } \\
\text { clear beam model names; disable } \\
\text { extended SSDs in other beam models. }\end{array}$ \\
\hline 11. Data transfer & $\begin{array}{l}\text { a. Corrupt R\&V data } \\
\text { especially with manual } \\
\text { data transfer }\end{array}$ & 4 & 2 & $\mathrm{H}$ & $\begin{array}{l}\text { Use TPS for data transfer to R\&V; Pre- } \\
\text { treatment check first } 10 \text { patients; use } \\
\text { templates if no TPS in involved. }\end{array}$ \\
\hline $\begin{array}{l}\text { 12. Large number of } \\
\text { MUs }\end{array}$ & $\begin{array}{l}\text { a. Not all beams or } \\
\text { segments are } \\
\text { created/registered/used }\end{array}$ & 4 & 2 & $\mathrm{H}$ & $\begin{array}{l}\text { Use TPS for data transfer to R\&V; } \\
\text { Surveillance and registration of } \\
\text { administered dose always in R\&V; use } \\
\text { templates if no TPS in involved. }\end{array}$ \\
\hline \multirow[t]{3}{*}{$\begin{array}{l}13 . \quad \text { Prolonged } \\
\text { treatment interruption }\end{array}$} & $\begin{array}{l}\text { a. Patient can get ill during } \\
\text { the treatment }\end{array}$ & 4 & 1 & $\mathrm{~L}$ & $\begin{array}{l}\text { Set up a dedicated TBI team and perform } \\
\text { an appropriate training on the } \\
\text { continuation of the treatment in } \\
\text { combination with } R \& V \text { system. }\end{array}$ \\
\hline & $\begin{array}{l}\text { b. Interruption due to linac } \\
\text { failure }\end{array}$ & 4 & 2 & $\mathrm{H}$ & $\begin{array}{l}\text { Since prolonged interruption of TBI may } \\
\text { severely compromise treatment } \\
\text { outcome a backup on-site is necessarily. }\end{array}$ \\
\hline & $\begin{array}{l}\text { c. No TBI treatment } \\
\text { machine available (also no } \\
\text { backup machine) due to } \\
\text { e.g. institutions power } \\
\text { failure or major upgrades } \\
\text { on the TBI treatment } \\
\text { machines }\end{array}$ & 4 & 2 & $\mathrm{H}$ & $\begin{array}{l}\text { Do not plan a } \mathrm{TBI} \text { procedure directly } \\
\text { before or after a planned emergency } \\
\text { power test at the institution or after } \\
\text { major TBI machine upgrades. }\end{array}$ \\
\hline \multirow[t]{4}{*}{ 14. In-vivo dosimetry } & a. Errors in measurement & 2 & 1 & $\mathrm{VL}$ & $\begin{array}{l}\text { Set up a dedicated TBI team; use an in- } \\
\text { vivo QA-system to check the calibration } \\
\text { values. }\end{array}$ \\
\hline & $\begin{array}{l}\text { b. Errors in positioning the } \\
\text { detectors }\end{array}$ & 2 & 2 & L & $\begin{array}{l}\text { Set up a dedicated TBI team; use } \\
\text { predefined positions of the detectors. }\end{array}$ \\
\hline & c. Measurement forgotten & 1 & 1 & $\mathrm{VL}$ & $\begin{array}{l}\text { Set up a dedicated } \mathrm{TBI} \text { team; use } \\
\text { reminders in } \mathrm{R} \& \mathrm{~V} \text {. }\end{array}$ \\
\hline & $\begin{array}{l}\text { d. Unexpected results due } \\
\text { to expired detector } \\
\text { calibration }\end{array}$ & 1 & 2 & $\mathrm{VL}$ & Check calibration periodically. \\
\hline
\end{tabular}

Table 6.2 Specific risks related to the sweeping TBI technique. With severities (S) ranging from minor (1) to catastrophic (4) (see definitions in Figure 6.2). Probability (P) ranging from 1 out of 100 patients (seldom) (1) to 1 out of 2 patients (4). Resulting risk scores (RS) Very Low (VL), Low (L), High (H) and Very High (VH). 


\begin{tabular}{|l|l|l|l|l|l|}
\hline Process description & $\begin{array}{l}\text { Possible failure mode / } \\
\text { consequence }\end{array}$ & S & P & RS & Possible solution \\
\hline $\begin{array}{l}\text { 15. Treatment based on } \\
\text { two CT scans (supine and } \\
\text { prone) }\end{array}$ & $\begin{array}{l}\text { a. Treatment is based on } \\
\text { thickness in one CT scan } \\
\text { instead on both CT-scans }\end{array}$ & 3 & 2 & L & Training \\
\hline $\begin{array}{l}\text { 16. Treatment based on } \\
\text { an adopted generic arc }\end{array}$ & $\begin{array}{l}\text { a. Generic arc is not } \\
\text { adopted to individual } \\
\text { patient }\end{array}$ & 4 & 1 & L & $\begin{array}{l}\text { Training and corrupting the generic } \\
\text { arc regarding one obvious parameter. }\end{array}$ \\
\hline
\end{tabular}

\subsubsection{Examples of failure modes in the TSI clinical workflow}

The following list gives specific items in the workflow for TSI and their possible failure modes. The numbers between brackets refer to the corresponding rows in Table 6.3.

- Multiple beams for large number of MUs

- wrong number of monitor units planned (17a)

- not all beams are created in the $R \& V$-system (18a)

- not all beams are administered in the R\&V-system (18a)

- not all beams are given (18a)

- Dedicated movable couch or positioning/fixation stand (often home-made)

- The couch can be positioned on a wrong SSD-position (19b)

- Extra positioning lasers

- The extra positioning lasers are not checked frequently (19a)

- Multiple patient positions

- Patient positioning is suboptimal (19c)

- Not all patient positions are irradiated (20a)

- Patient positions are irradiated more than once (20b)

- Use of beam scatterer - energy degrader

- beam scatterer - energy degrader is not used (21a)

- Wrong position

- Wrong dimensions (21b) 
Table 6.3 Risk analysis for TSI treatment. Severities (S) ranging from minor (1) to catastrophic (4) (see definitions above). Probability (P) ranging from 1 out of 100 patients (seldom) (1) to 1 out of 2 patients (4). Resulting risk scores (RS): Very Low (VL), Low (L), High (H) and Very High (VH).

\begin{tabular}{|c|c|c|c|c|c|}
\hline Process description & $\begin{array}{l}\text { Possible failure mode / } \\
\text { consequence }\end{array}$ & $\mathbf{S}$ & $\mathbf{P}$ & RS & Possible solution \\
\hline $\begin{array}{l}\text { 17. Large Number of } \\
\text { MUs due to extended } \\
\text { SSD }\end{array}$ & $\begin{array}{l}\text { a. Planning wrong } \\
\text { number of MUs due to } \\
\text { unfamiliarity with TSI }\end{array}$ & 4 & 2 & $\mathrm{H}$ & $\begin{array}{l}\text { Create a TSI template in R\&V; set up a } \\
\text { dedicated TSI team. }\end{array}$ \\
\hline 18. Multiple beams & $\begin{array}{l}\text { a. Not all beams } \\
\text { created/administered }\end{array}$ & 3 & 2 & $\mathrm{~L}$ & $\begin{array}{l}\text { Create a TSI template in R\&V with well-chosen } \\
\text { beam names, include patient position remarks } \\
\text { in field notes; Set up a dedicated TSI team. }\end{array}$ \\
\hline \multirow[t]{3}{*}{ 19. Patient positioning } & $\begin{array}{l}\text { a. Wrong positioning } \\
\text { due to offset TSI laser(s) }\end{array}$ & 2 & 1 & VL & $\begin{array}{l}\text { Regular QA or } 100 \% \text { pre-treatment } \mathrm{QA} \text { on } \\
\text { position lasers for } \mathrm{TSI} \text {. }\end{array}$ \\
\hline & $\begin{array}{l}\text { b. Wrong SSD TSI stand/ } \\
\text { fixation }\end{array}$ & 3 & 1 & $\mathrm{VL}$ & TSI laser; Set up a dedicated TSI team. \\
\hline & $\begin{array}{l}\text { c. Dose failure due to } \\
\text { suboptimal patient } \\
\text { positioning in stand }\end{array}$ & 3 & 3 & $\mathrm{H}$ & $\begin{array}{l}\text { Use patient positioning remarks in the field } \\
\text { notes; set up a dedicated TSI team. }\end{array}$ \\
\hline \multirow[t]{2}{*}{$\begin{array}{l}\text { 20. Multiple patient } \\
\text { positions }\end{array}$} & $\begin{array}{l}\text { a. Not all positions are } \\
\text { used }\end{array}$ & 3 & 2 & $L$ & $\begin{array}{l}\text { Beam name and position should have } \\
\text { traceable connection. }\end{array}$ \\
\hline & $\begin{array}{l}\text { b. Positions are doubly } \\
\text { irradiated }\end{array}$ & 3 & 2 & $L$ & $\begin{array}{l}\text { Beam name and position should have } \\
\text { traceable connection. }\end{array}$ \\
\hline \multirow[t]{2}{*}{$\begin{array}{l}\text { 21. Beam scatterer/ } \\
\text { energy degrader }\end{array}$} & $\begin{array}{l}\text { a. Dose failure due to } \\
\text { TSI without scatterer }\end{array}$ & 4 & 2 & $\mathrm{H}$ & $\begin{array}{l}\text { Use } 4 \text { MeV avoiding necessity scatterer; set up } \\
\text { a dedicated TSI team.. }\end{array}$ \\
\hline & $\begin{array}{l}\text { b. TSI with wrong } \\
\text { scatterer }\end{array}$ & 3 & 1 & $\mathrm{~L}$ & $\begin{array}{l}\text { Use } 4 \mathrm{MeV} \text { avoiding necessity scatterer; if } \\
\text { required label the scatterer and keep only } 1 \\
\text { scatter in the department. }\end{array}$ \\
\hline
\end{tabular}

\subsection{Recommendations}

Process steps resulting in either High or Very High risks scores should ideally be avoided or eliminated, or at least be minimized.

In the recommendation below, measures are summarised based on the solutions mentioned in Table 6.1, Table 6.2 and Table 6.3. The numbers between brackets refer to the numbers for the process \& failure modes in these tables.

\section{TBI-recommendations}

In case of TBI, the following measures should be taken:

i) set up a dedicated TBI team that is familiar and trained periodically with the treatment execution and recognizes abnormalities (3c, 8b),

ii) Dose calculation and data transfer to linac:

a. use of CT based planning with the use of scripting possibilities of the TPS (9a, 9b), 
b. use templates for non TPS procedures (11a, 12a),

iii) use the R\&V system with automated TPS - R\&V data transfer (3b, 9a, 11a, 12a),

iv) when dedicated positioning devices (e.g. movable couch) are used:

a. these devices should be robust and checked periodically (3c),

b. have maximal as possible patient comfort (8b),

c. have verification tools (such as read out positions) and use skin marks (3b, $8 a)$,

v) have a backup TBI capable linac on site (preferably with matched beam and vault geometry to avoid mistakes when going over) (13b),

vi) do not plan a TBI procedure directly before or after an institution power emergency test or after major TBI machine upgrades (13c).

\section{TSI-recommendations}

In the case of TSI the following measures are suggested:

i) set up a dedicated TSI team that is familiar and trained periodically with the treatment execution and recognizes abnormalities; such a team is crucial for optimal patient positioning; use patient positioning remarks in the field notes (19c, 21a),

ii) create a template for TSI treatment in the R\&V to avoid incomplete treatments (17a),

iii) apply $4 \mathrm{MeV}$ to avoid the necessity of a scatterer/ energy degrader. (21a)

The above-mentioned measures should avoid most severe errors classified with either High or Very High risks, provided the complete TBI / TSI treatment chain is properly commissioned. To check the class solution and whether the personnel is trained adequately we recommend pre-treatment runs and end-to-end tests combined with in-vivo checks at least for the first 10 treatments. In case the number of TBI / TSI patients per year is low ( $<5$ per year), the dedicated team should not be too large and the team should be trained periodically. 


\section{Final words and some remarks about future developments}

This part of the report describes a possible development for the TBI/TSI treatments in the future. As such, it serves as a guidance towards a new level of the state-of-the-art treatment technique. The state of the art for a Total Body or Total Skin Irradiation implies imaging modalities in treatment position, allowing the visualization of the parts of the body to be treated and the organs at risk to be spared. For the Total Body Irradiation, the treatment planning system is adapted for the treatment modality chosen (large SSD, arc technique, sweeping gap technique) and using inverse planning the prescription for the planning target volume and dose reduction for the organs at risk is obtained. Intensity modulated beams are generated and sent to the record and verify system.

For Total Skin Irradiation, Monte Carlo based dose engines will provide the needed accuracy for the dose prediction of superposition of multiple electron beams at large SSD, allowing MU optimization. Moreover, the treatment planning system will generate an RTplan that is used to feed the record and verify system avoiding manual data entry.

Prior to the TBI treatment patient positioning is validated using flat panels in the room behind the patient and transit dosimetry is used to validate the patient position during the entire treatment and to validate the registered dose on the patient. Online position corrections are possible using remote controlled treatment stands/tables. As alternative video-assisted (infrared) position verification is used in order to allow patient positioning verification during the entire treatment when small treatment field sizes and therefore limited field of view due to modulation of the beams is limiting accurate positioning verification using the MV beams. In vivo dosimetry will keep its role to check new treatment techniques and verify the consequences of posture changes during treatment.

The same video-assisted position verification may be used for patient positioning verification in TSI and combined with the Monte Carlo Dose prediction; the main purpose of the in vivo results will be limited to treatment verification. 


\section{Bibliography}

[1] Dessauer F. Eine neue anordnung zur Röntgenbestrahlung. Arch Für Phys Medizin Und Medizinische Tech 1907;2:218-23.

[2] Dessauer F. Beiträge zur Bestrahlung tiefliegender Prozesse. Medizinischen Klin 1905;21.

[3] Elfer A. Orvosi Hetilap 1907.

[4] Medinger F, Craver L. Total body irradiation with review of cases. Am J Roentgenol Radium Ther Nucl Med 1942;48:651-671.

[5] Hoppe R, Phillips T, Roach M. Chapter 15: total body irradiation. Leibel Phillips Textb. Radiat. Oncol. 3rd ed., 2010.

[6] Miller LS, Fletcher GH, Gerstner HB. Radiobiologic observations on cancer patients treated with wholebody x-irradiation. Radiat Res 1958;8:150-65.

[7] Collins VP, Loeffler RK. The therapeutic use of single doses of total body radiation. Am J Roentgenol Radium Ther Nucl Med 1956;75:542-7.

[8] Saenger EL, Silberstein EB, Aron B, Horwitz H, Kereiakes JG, Bahr GK, et al. Whole body and partial body radiotherapy of advanced cancer. Am J Roentgenol Radium Ther Nucl Med 1973;117:670-85. doi:10.2214/ajr.117.3.670.

[9] Energy UD of. Advisory Committee on Human Radiation Experiments: Final Report. Washington DC: 1995.

[10] Merrill JP, Murray JE, Harrison JH, Guild WR. Successful homotransplantation of the human kidney between identical twins. J Am Med Assoc 1956;160:277-82. doi:10.1001/jama.1956.02960390027008.

[11] Murray JE, Merrill JP, Harrison JH, Wilson RE, Dammin GJ. Prolonged survival of human-kidney homografts by immunosuppressive drug therapy. N Engl J Med 1963;268:1315-23. doi:10.1056/NEJM196306132682401.

[12] Thomas ED, Lochte HL, Lu WC, Ferrebee JW. Intravenous infusion of bone marrow in patients receiving radiation and chemotherapy. N Engl J Med 1957;257:491-6. doi:10.1056/NEJM195709122571102.

[13] Thomas ED, Lochte HLJ, Ferrebee JW. Irradiation of the entire body and marrow transplantation: some observations and comments. Blood 1959;14:1-23.

[14] Buckner CD, Epstein RB, Rudolph RH, Clift RA, Storb R, Thomas ED. Allogeneic marrow engraftment following whole body irradiation in a patient with leukemia. Blood 1970;35:741-50.

[15] Thomas E, Storb R, Clift RA, Fefer A, Johnson FL, Neiman PE, et al. Bone-marrow transplantation (first of two parts). N Engl J Med 1975;292:832-43. doi:10.1056/NEJM197504172921605.

[16] Thomas ED, Storb R, Clift RA, Fefer A, Johnson L, Neiman PE, et al. Bone-marrow transplantation (second of two parts). N Engl J Med 1975;292:895-902. doi:10.1056/NEJM197504242921706.

[17] UMC Groningen. Autologe stamceltransplantatie (Verrichtingen en algemene (niet-ziektegebonden) behandelingen) 2020.

[18] UMC Groningen. Allogene stamceltransplantatie (Verrichtingen en algemene (niet-ziektegebonden) behandelingen) 2020.

[19] Barrett A. Total body irradiation before bone marrow transplantation: a review. Clin Radiol 1982;33:1315. doi:10.1016/s0009-9260(82)80035-4.

[20] Wong JYC, Filippi AR, Dabaja BS, Yahalom J, Specht L. Total Body Irradiation: Guidelines from the International Lymphoma Radiation Oncology Group (ILROG). Int J Radiat Oncol 2018;101:521-9. doi:10.1016/j.ijrobp.2018.04.071.

[21] Chaillet MP, Cosset JM, Socie G, Pico JL, Grimaud E, Dubray B, et al. Prospective study of the clinical symptoms of therapeutic whole body irradiation. Health Phys 1993;64:370-4. doi:10.1097/00004032199304000-00003.

[22] Buchali A, Feyer P, Groll J, Massenkeil G, Arnold R, Budach V. Immediate toxicity during fractionated total body irradiation as conditioning for bone marrow transplantation. Radiother Oncol J Eur Soc Ther Radiol Oncol 2000;54:157-62. doi:10.1016/s0167-8140(99)00178-4.

[23] Ozsahin M, Pène F, Touboul E, Gindrey-Vie B, Dominique C, Lefkopoulos D, et al. Total-body irradiation before bone marrow transplantation. Results of two randomized instantaneous dose rates in 157 patients. Cancer 1992;69:2853-65. doi:10.1002/1097-0142(19920601)69:11<2853::aidcncr2820691135>3.0.co;2-2.

[24] Smith TJ, Khatcheressian J, Lyman GH, Ozer H, Armitage JO, Balducci L, et al. 2006 Update of Recommendations for the Use of White Blood Cell Growth Factors: An Evidence-Based Clinical Practice 
Guideline. J Clin Oncol 2006;24:3187-205. doi:10.1200/JCO.2006.06.4451.

[25] Ping Y Torres JL, Bross DS, Lam W, Wharam MD, Santos GW, Order SE. Risk factors in interstitial pneumonitis following allogenic bone marrow transplantation. Int J Radiat Oncol 1982;8:1301-7. doi:10.1016/0360-3016(82)90579-X.

[26] Weiner RS. Interstitial Pneumonitis After Bone Marrow Transplantation. Ann Intern Med 1986;104:168. doi:10.7326/0003-4819-104-2-168.

[27] Bortin M, Kay HM, Peter Gale R, Rimm A. Factors Associated with Interstitial Pneumonitis ffter BoneMarrow Transplantation for Acute Leukaemia. Lancet 1982;319:437-9. doi:10.1016/S01406736(82)91633-6.

[28] Deeg HJ, Sullivan KM, Buckner CD, Storb R, Appelbaum FR, Clift RA, et al. Marrow transplantation for acute nonlymphoblastic leukemia in first remission: toxicity and long-term follow-up of patients conditioned with single dose or fractionated total body irradiation. Bone Marrow Transplant 1986;1:1517.

[29] Girinsky T, Benhamou E, Bourhis J-H, Dhermain F, Guillot-Valls D, Ganansia V, et al. Prospective Randomized Comparison of Single-Dose Versus Hyperfractionated Total-Body Irradiation in Patients With Hematologic Malignancies. J Clin Oncol 2000;18:981-981. doi:10.1200/JCO.2000.18.5.981.

[30] Morgan TL, Falk PM, Kogut N, Shah KH, Tome M, Kagan AR. A comparison of single-dose and fractionted total-body irradiation on the development of pneumonitis following bone marrow transplantation. Int J Radiat Oncol 1996;36:61-6. doi:10.1016/S0360-3016(96)00246-5.

[31] Tarbell NJ, Amato DA, Down JD, Mauch P, Hellman S. Fractionation and dose rate effects in mice: A model for bone marrow transplantation in man. Int J Radiat Oncol 1987;13:1065-9. doi:10.1016/03603016(87)90046-0.

[32] Travis EL, Peters LJ, McNeill J, Thames HD, Karolis C. Effect of dose-rate on total body irradiation: Lethality and pathologic findings. Radiother Oncol 1985;4:341-51. doi:10.1016/S0167-8140(85)80122-5.

[33] Tichelli A, Passweg J, Wojcik D, Rovo A, Harousseau J-L, Masszi T, et al. Late cardiovascular events after allogeneic hematopoietic stem cell transplantation: a retrospective multicenter study of the Late Effects Working Party of the European Group for Blood and Marrow Transplantation. Haematologica 2008;93:1203-10. doi:10.3324/haematol.12949.

[34] Armenian SH, Sun C-L, Francisco L, Steinberger J, Kurian S, Wong FL, et al. Late Congestive Heart Failure After Hematopoietic Cell Transplantation. J Clin Oncol 2008;26:5537-43. doi:10.1200/JCO.2008.17.7428.

[35] Ferrara JLM, Levine JE, Reddy P, Holler E. Graft-versus-host disease. Lancet (London, England) 2009;373:1550-61. doi:10.1016/S0140-6736(09)60237-3.

[36] Filipovich AH, Weisdorf D, Pavletic S, Socie G, Wingard JR, Lee SJ, et al. National Institutes of Health Consensus Development Project on Criteria for Clinical Trials in Chronic Graft-versus-Host Disease: I. Diagnosis and Staging Working Group Report. Biol Blood Marrow Transplant 2005;11:945-56. doi:10.1016/j.bbmt.2005.09.004.

[37] Nakasone H, Fukuda T, Kanda J, Mori T, Yano S, Kobayashi T, et al. Impact of conditioning intensity and TBI on acute GVHD after hematopoietic cell transplantation. Bone Marrow Transplant 2015;50:559-65. doi:10.1038/bmt.2014.293.

[38] Quast U. Whole body radiotherapy: A TBI-guideline. J Med Phys 2006;31:5-12. doi:10.4103/09716203.25664 .

[39] Trump JG, Wright KA, Evans WW, Anson JH, Hare HF, Fromer JL, et al. High energy electrons for the treatment of extensive superficial malignant lesions. Am J Roentgenol Radium Ther Nucl Med 1953;69:623-9.

[40] Karzmark CJ, Loevinger R, Steele RE, Weissbluth M. A Technique for Large-Field, Superficial Electron Therapy. Radiology 1960;74:633-44. doi:10.1148/74.4.633.

[41] Page V, Gardner A, Karzmark CJ. Patient dosimetry in the electron treatment of large superficial lesions. Radiology 1970;94:635-41. doi:10.1148/94.3.635.

[42] Smedal MI, Johnston DO, Salzman FA, Trump JG, Wright KA. Ten year experience with low megavolt ellectron therapy. Am J Roentgenol Radium Ther Nucl Med 1962;88:215-28.

[43] Hoppe RT, Fuks Z, Bagshaw MA. Radiation therapy in the management of cutaneous T-cell lymphomas. Cancer Treat Rep 1979;63:625-32.

[44] Karzmark CJ, Anderson J, Buffa A, Fessenden P, Khan F, Svensson G, et al. Total Skin Electron Therapy: Technique and Dosimetry. 1987. doi:10.37206/22.

[45] ICRU. Report 48. Phantoms and Computational Models in Therapy, Diagnosis and Protection. Bethesda, 
MD, USA: 1992.

[46] Hoppe RT. Mycosis fungoides: Radiation therapy. Dermatol Ther 2003;16:347-54. doi:10.1111/j.13960296.2003.01647.x.

[47] Specht L, Dabaja B, Illidge T, Wilson LD, Hoppe RT. Modern radiation therapy for primary cutaneous lymphomas: Field and dose guidelines from the international lymphoma radiation oncology group. Int J Radiat Oncol Biol Phys 2015;92:32-9. doi:10.1016/j.ijrobp.2015.01.008.

[48] Lindahl LM, Kamstrup MR, Petersen PM, Wirén J, Fenger-Grøn M, Gniadecki R, et al. Total skin electron beam therapy for cutaneous T-cell lymphoma: A nationwide cohort study from Denmark. Acta Oncol (Madr) 2011;50:1199-205. doi:10.3109/0284186X.2011.585999.

[49] Kamstrup MR, Gniadecki R, Iversen L, Skov L, Petersen PM, Loft A, et al. Low-dose (10-Gy) total skin electron beam therapy for cutaneous t-cell lymphoma: An open clinical study and pooled data analysis. Int J Radiat Oncol Biol Phys 2015;92:138-43. doi:10.1016/j.ijrobp.2015.01.047.

[50] Mazzeo E, Rubino L, Buglione M, Antognoni P, Magrini SM, Bertoni F, et al. The current management of mycosis fungoides and Sézary syndrome and the role of radiotherapy: Principles and indications. Reports Pract Oncol Radiother 2014;19:77-91. doi:10.1016/j.rpor.2013.07.009.

[51] Moraes FY de, Carvalho H de A, Hanna SA, Silva JLF da, Marta GN. Literature review of clinical results of total skin electron irradiation (TSEBT) of mycosis fungoides in adults. Reports Pract Oncol Radiother 2014;19:92-8. doi:10.1016/j.rpor.2013.08.003.

[52] Dault JB, Slade AN, Zhao S, Song S. Comparison of low-dose and standard-dose total skin electron beam therapy in mycosis fungoides. Leuk Lymphoma 2019;60:2334-6. doi:10.1080/10428194.2019.1574008.

[53] Koken PW, Murrer L. Total Body Irradiation and Total Skin Irradiation techniques in Belgium and the Netherlands - current clinical practice. Adv Radiat Oncol 2021:100664. doi:10.1016/j.adro.2021.100664.

[54] Barret A. Total Body Irradiation. Reports Pract Oncol Radiother 1999;4:47-64.

[55] Mathé G, Amiel JL, Schwarzenberg L, Cattan A, Schneider M. Adoptive Immunotherapy of Acute Leukemia: Experimental and Clinical Results. Cancer Res 1965;25:1525-31.

[56] Hill-Kayser CE, Plastaras JP, Tochner Z, Glatstein E. TBI during BM and SCT: Review of the past, discussion of the present and consideration of future directions. Bone Marrow Transplant 2011;46:475-84. doi:10.1038/bmt.2010.280.

[57] Quast U. Total body irradiation- review of treatment techniques in Europe * 1987;9:91-106.

[58] Inoue T, Mori T, lino Y, Sugawara T, Masaoka T, Shibata H. National Survey of Bone Marrow Transplantation and Total Body Irradiation With Special Reference to Treatment Schedule in Japan 1989;119.

[59] Studinski RCN, Fraser DJ, Samant RS, MacPherson MS. Current practice in total-body irradiation: results of a Canada-wide survey. Curr Oncol 2017;24:181. doi:10.3747/co.24.3484.

[60] Ishibashi N, Soejima T, Kawaguchi H, Oguchi M, Sasai K, Hasegawa M, et al. National Survey of Japan for Myeloablative Total Body Irradiation Prior to Hematopoietic Stem Cell Transplantation. Int J Radiat Oncol 2017;99:E429. doi:10.1016/j.ijrobp.2017.06.1630.

[61] Ishibashi N, Soejima T, Kawaguchi H, Akiba T, Hasegawa M, Isobe K, et al. National survey of myeloablative total body irradiation prior to hematopoietic stem cell transplantation in Japan: survey of the Japanese Radiation Oncology Study Group (JROSG). J Radiat Res 2018;59:477-83. doi:10.1093/jrr/rry017.

[62] Giebel S, Miszczyk L, Slosarek K, Moukhtari L, Ciceri F, Esteve J, et al. Extreme heterogeneity of myeloablative total body irradiation techniques in clinical practice: A survey of the acute leukemia working party of the european group for blood and marrow transplantation. Cancer 2014;120:2760-5. doi:10.1002/cncr.28768.

[63] Nelligan R, Bailey M, Tran T, Baldwin Z. ACPSEM ROSG TBI working group recommendations for quality assurance in total body irradiation. Australas Phys Eng Sci Med 2015;38:205-15. doi:10.1007/s13246015-0344-7.

[64] Fog LS, Wirth A, MacManus M, Downes S, Grace M, Moggré A, et al. Total body irradiation in Australia and New Zealand: results of a practice survey. Phys Eng Sci Med 2020;43:825-35. doi:10.1007/s13246020-00878-z.

[65] Holmes T, Das R, Low D, Yin F-F, Balter J, Palta J, et al. American Society of Radiation Oncology Recommendations for Documenting Intensity-Modulated Radiation Therapy Treatments. Int J Radiat Oncol 2009;74:1311-8. doi:10.1016/j.ijrobp.2009.04.037.

[66] Das IJ, Galavis P, Mistry N, Hitchen C, Gerber NK. Total Body Irradiation Techniques: Patterns of Care with 
Advanced Technology. Int J Radiat Oncol 2018;102:e489. doi:10.1016/j.ijrobp.2018.07.1392.

[67] Piotrowski T, Milecki P, Skórska M, Fundowicz D. Total skin electron irradiation techniques: A review. Postep Dermatologii i Alergol 2013;30:50-5. doi:10.5114/pdia.2013.33379.

[68] Fogliata A, Cozzi L, Clivio A, Ibatici A, Mancosu P, Navarria P, et al. Preclinical assessment of volumetric modulated arc therapy for total marrow irradiation. Int J Radiat Oncol Biol Phys 2011;80:628-36. doi:10.1016/j.ijrobp.2010.11.028.

[69] Han C, Schultheisss TE, Wong JYC. Dosimetric study of volumetric modulated arc therapy fields for total marrow irradiation. Radiother Oncol 2012;102:315-20. doi:10.1016/j.radonc.2011.06.005.

[70] Jahnke A, Jahnke L, Molina-Duran F, Ehmann M, Kantz S, Steil V, et al. Arc therapy for total body irradiation - A robust novel treatment technique for standard treatment rooms. Radiother Oncol 2014;110:553-7. doi:10.1016/j.radonc.2013.12.009.

[71] Kirby N, Held M, Morin O, Fogh S, Pouliot J. Inverse-planned modulated-arc total-body irradiation 2012;1708:2761-4.

[72] Wong JYC, Liu A, Schultheiss T, Popplewell L, Stein A, Rosenthal J, et al. Targeted total marrow irradiation using three-dimensional image-guided tomographic intensity-modulated radiation therapy: An alternative to standard total body irradiation. Biol Blood Marrow Transplant 2006;12:306-15. doi:10.1016/j.bbmt.2005.10.026.

[73] Wong JYC, Rosenthal J, Liu A, Schultheiss T, Forman S, Somlo G. Image-Guided Total-Marrow Irradiation Using Helical Tomotherapy in Patients With Multiple Myeloma and Acute Leukemia Undergoing Hematopoietic Cell Transplantation. Int J Radiat Oncol Biol Phys 2009;73:273-9. doi:10.1016/j.ijrobp.2008.04.071.

[74] Zhuang AH, Liu A, Schultheiss TE, Wong JYC. Dosimetric Study and Verification of Total Body Irradiation Using Helical Tomotherapy and its Comparison to Extended SSD Technique. Med Dosim 2010;35:243-9. doi:10.1016/j.meddos.2009.07.001.

[75] Evans S, Christofides S, Brambilla M. The European Federation of Organisations for Medical Physics. Policy Statement No. 7.1: The roles, responsibilities and status of the medical physicist including the criteria for the staffing levels in a Medical Physics Department approved by EFOMP Counci. Phys Medica 2016;32:533-40. doi:10.1016/j.ejmp.2016.03.001.

[76] Peñagarícano JA, Chao M, Van Rhee F, Moros EG, Corry PM, Ratanatharathorn V. Clinical feasibility of TBI with helical tomotherapy. Bone Marrow Transplant 2011;46:929-35. doi:10.1038/bmt.2010.237.

[77] Hudson A, Gordon D, Moore R, Balogh A, Pierce G. Sci-Thur PM - Colourful Interactions: Highlights 08: ARC TBI using Single-Step Optimized VMAT Fields. Med Phys 2016;43:4933-4933. doi:10.1118/1.4961768.

[78] Barbet J, Chatal J, Kraeber-bodéré F. Les anticorps radiomarqués pour le traitement des cancers 2009.

[79] Bethge WA, Lange T, Meisner C, Von Harsdorf S, Bornhaeuser M, Federmann B, et al. Radioimmunotherapy with yttrium-90-ibritumomab tiuxetan as part of a reduced-intensity conditioning regimen for allogeneic hematopoietic cell transplantation in patients with advanced non-Hodgkin lymphoma: Results of a phase 2 study. Blood 2010;116:1795-802. doi:10.1182/blood-2010-02-270538.

[80] Zoller W. Organ-Sparing Marrow-Targeted Irradiation as an Alternative to Traditional Total Body Irradiation Methods: VMAT Treatment Planning and Clinical Implementation. VarianWebinar 2017. https://varian.force.com/apex/CpWebSummary?id=a004400000rl2UQEAY (accessed June 10, 2020).

[81] Losert C, Shpani R, Kießling R, Freislederer P, Li M, Walter F, et al. Novel rotatable tabletop for total-body irradiation using a linac-based VMAT technique. Radiat Oncol 2019;14:1-10. doi:10.1186/s13014-0191445-3.

[82] Dutch cancer registry. Dutch cancer registry (IKNL) databases 2018:https://iknl.nl/nkr-cijfers.

[83] Belgium Cancer Registry. Cancer Incidence in Belgium 2012. kankerregister.org/media/docs/publications/HaematologicalMalignancies2012.pdf.

[84] Van Leeuwen RGH, Murrer LHP, Ta BDP, Lotz HT, Beijert M, Schaap NPM, et al. Total Body Irradiation (TBI) in hematopoietic stem cell transplantation: a profession in its own right. Ned Tijdschr v Hematol 2019;16:343-8.

[85] Van Dyk J, American Institute of Physics., American Association of Physicists in Medicine. The physical aspects of total and half body photon irradiation. 1986.

[86] DeRosier J, Stalhandske E, Bagian JP, Nudell T. Using health care Failure Mode and Effect Analysis: the VA National Center for Patient Safety's prospective risk analysis system. Jt Comm J Qual Improv 2002;28:248-67. doi:10.1016/s1070-3241(02)28025-6. 
[87] VMS-Zorg. Praktijkgids Prospectieve Risico-Inventarisatie ( PRI ) 2012:58.

[88] Diamantopoulos S, Platoni K, Dilvoi M, Nazos I, Geropantas K, Maravelis G, et al. Clinical implementation of total skin electron beam (TSEB) therapy: A review of the relevant literature. Phys Medica 2011;27:628. doi:10.1016/j.ejmp.2010.09.001.

[89] Hoeben BAW, Pazos M, Albert MH, Seravalli E, Bosman ME, Losert C, et al. Towards homogenization of total body irradiation practices in pediatric patients across SIOPE affiliated centers. A survey by the SIOPE radiation oncology working group. Radiother Oncol 2021;155:113-9. doi:10.1016/j.radonc.2020.10.032.

[90] NVZ, NFU, RN. Convenant Veilige Toepassing van medische technologie in het ziekenhuis. 2011.

[91] Shank B. Toxicity due to total body irradiation. Hum. Radiat. Inj., 2011.

[92] Graves SS, Storer BE, Butts TM, Storb R. Comparing high and low total body irradiation dose rates for minimum-intensity conditioning of dogs for dog leukocyte antigen-identical bone marrow grafts. Biol Blood Marrow Transplant 2013;19:1650-4. doi:10.1016/j.bbmt.2013.08.007.

[93] Fog LS, Hansen VN, Kjær-Kristoffersen F, Berlon TE, Petersen PM, Mandeville H, et al. A step and shoot intensity modulated technique for total body irradiation. Tech Innov Patient Support Radiat Oncol 2019;10:1-7. doi:10.1016/j.tipsro.2019.05.002.

[94] Bloemen-van Gurp EJ, Mijnheer BJ, Verschueren TAMM, Lambin P. Total Body Irradiation, Toward Optimal Individual Delivery: Dose Evaluation With Metal Oxide Field Effect Transistors, Thermoluminescence Detectors, and a Treatment Planning System. Int J Radiat Oncol Biol Phys 2007;69:1297-304. doi:10.1016/j.ijrobp.2007.07.2334.

[95] Peters M, Taylor B, Turner E. An Evidence-Based Review of Total Body Irradiation. J Med Imaging Radiat Sci 2015;46:442-9. doi:10.1016/j.jmir.2015.09.007.

[96] Zhuang T, Wu Q. Generating arbitrary one-dimensional dose profiles using rotational therapy. Phys Med Biol 2010;55:6263-77. doi:10.1088/0031-9155/55/20/014.

[97] Springer A, Hammer J, Winkler E, Track C, Huppert R, Böhm A, et al. Total body irradiation with volumetric modulated arc therapy: Dosimetric data and first clinical experience. Radiat Oncol 2016;11:1-9. doi:10.1186/s13014-016-0625-7.

[98] Ramm U, Licher J, Moog J, Scherf C, Kara E, Böttcher HD, et al. In vivo dosimetry with semiconducting diodes for dose verification in total-body irradiation: A 10-year experience. Strahlentherapie Und Onkol 2008;184:376-80. doi:10.1007/s00066-008-1823-5.

[99] Aalbers AHL, Hoornaert M-T, Minken A, Palmans H, Pieksma MWH, De Prez LA, et al. NCS Report 18: Code of practice for the absorbed dose determination in high energy photon and electron beams. Delft: 2008. doi:10.25030/ncs-018.

[100] van Leeuwen RGH, Verwegen D, van Kollenburg PGM, Swinkels M, van der Maazen RWM. Early clinical experience with a total body irradiation technique using field-in-field beams and on-line image guidance. Phys Imaging Radiat Oncol 2020;16:12-7. doi:10.1016/j.phro.2020.09.004.

[101] Pla M, Chenery SG, Podgorsak EB. Total body irradiation with a sweeping beam. Int J Radiat Oncol 1983;9:83-9. doi:10.1016/0360-3016(83)90214-6.

[102] Polednik M, Lohr F, Ehmann M, Wenz F. Accelerating total body irradiation with large field modulated arc therapy in standard treatment rooms without additional equipment. Strahlentherapie Und Onkol 2015;191:869-74. doi:10.1007/s00066-015-0883-6.

[103] Bruinvis IAD, Keus RB, Lenglet WJM, Meijer GJ, Mijnheer BJ, Van 't Veld AA, et al. NCS Report 15: Quality assurance of 3-D treatment planning systems for external photon and electron beams. vol. 15. Delft: 2005. doi:10.25030/ncs-015.

[104] Rawlinson JA, Arlen D, Newcombe D. Design of parallel plate ion chambers for buildup measurements in megavoltage photon beams. Med Phys 1992;19:641-8. doi:10.1118/1.596896.

[105] Velkley DE, Manson DJ, Purdy JA, Oliver GD. Build-up region of megavoltage photon radiation sources. Med Phys 1975;2:14-9. doi:10.1118/1.594158.

[106] British Institute of Radiology. Central Axis Depth Dose Data for Use in Radiotherapy. Suppl. 25. London: British Journal of Radiology; 1996.

[107] Van der Wal E, Wiersma J, Ausma AH, Cuijpers JP, Tomsej M, Bos L, et al. NCS Report 22: Code of practice for the quality assurance and control for intensity modulated radiotherapy. Delft: 2013. doi:10.25030/ncs-022.

[108] Miften M, Mihailidis D, Kry SF, Reft C, Esquivel C, Farr J, et al. Management of radiotherapy patients with implanted cardiac pacemakers and defibrillators: A Report of the AAPM TG-203 †. Med Phys 2019;46:e757-88. doi:10.1002/mp.13838. 
[109] ACR-ASTRO. ACR-ASTRO Practice Parameter for the Performance of Total Body Irradiation, 2011.

[110] Onal C, Sonmez A, Arslan G, Sonmez S, Efe E, Oymak E. Evaluation of field-in-field technique for total body irradiation. Int J Radiat Oncol Biol Phys 2012;83:1641-8. doi:10.1016/j.ijrobp.2011.10.045.

[111] Guidi G, Gottardi G, Ceroni P, Costi T. Review of the results of the in vivo dosimetry during total skin electron beam therapy. Reports Pract Oncol Radiother 2014;19:144-50. doi:10.1016/j.rpor.2013.07.011.

[112] Wikipedia. BIV-classificatie 2020. https://nl.wikipedia.org/wiki/BIV-classificatie. [113] Wikipedia. Information security
https://en.wikipedia.org/wiki/Information_security\#Key_concepts.

2020. 


\section{Acknowledgements}

Finally, we want to acknowledge the persons involved in the drafting of this report: Maxime Coevoet, Nicolas Hermand, Heleen van Herpt, Loes van Kempen and Françoise Vanneste. Their input during the active period of this working group was greatly appreciated.

The members of the subcommittee would like to thank the external reviewers: Patrick Granton and Tom Boterberg for their valuable and extensive comments. 